\title{
BUNDLE THEORIES FOR TOPOLOGICAL MANIFOLDS
}

\author{
C. B. HUGHES, L. R. TAYLOR AND E. B. WILLIAMS
}

\begin{abstract}
Manifold approximate fibrations arise in the geometric topology of manifolds and group actions on topological manifolds. The primary purpose of this paper is to classify manifold approximate fibrations in terms of the lifting problem for a certain bundle. Our classification meshes well with the classical classifications of fibrations and bundles and, hence, we are able to attack questions such as the following. When is a fibration controlled homotopy equivalent to a manifold approximate fibration? When is a manifold approximate fibration controlled homeomorphic to a bundle?
\end{abstract}

Let $B^{i}$ be a topological manifold. Recall that a manifold approximate fibration over $B$ is a proper map $q: M \rightarrow B$ such that $M$ is a manifold (topological or Hilbert cube) and such that $q$ satisfies an approximate lifting condition (see [8] or $§ 1 . D)$. This "bundle" theory plays an important role in the study of topological manifolds. Consider the following examples.

Embedding theory. Edwards [13] and Quinn [31] have shown that a locally flat submanifold $B \subset V \quad(\operatorname{dim} V \geq 6)$ has a mapping cylinder neighborhood $M(q)$, where $q: M \rightarrow B$ is a manifold approximate fibration. Quinn [31] and Chapman [6] have applied approximate fibration theory to give local homotopical criteria for local flatness.

Group actions on topological manifolds. Quinn [32], [34] and [35] has used this theory to study group actions.

Existence of homeomorphisms. Suppose $q: E^{n+i} \rightarrow B$ is a fibre bundle with manifold fibre $F, n+i \geq 5$, and suppose $f: N^{n+i} \rightarrow E^{n+i}$ is a homotopy equivalence. If $q \circ f$ is an approximate fibration, then the obstruction to deforming $f$ to a homeomorphism can be expressed in terms of surgery theory on $F \times$ tori (see Chapman [6], Farrell-Hsiang [14], Hughes [20], and §1). Farrell and Hsiang have applied this idea to prove the Novikov conjecture for certain groups. They were able to use differential geometry to verify that the map $q \circ f$ was an approximate fibration in their case.

Received by the editors September 30, 1988.

1980 Mathematics Subject Classification. Primary 57N15, 55R15, 55R65; Secondary 55R05, 55R10, 57N20.

Key words and phrases. Manifold approximate fibration, fibration, bundle, controlled structures.

Partially supported by the N.S.F. 
In this paper we give a classification of manifold approximate fibrations which is analogous to the classical classification of fibrations and fibre bundles. Indeed, we repeat both those classifications here. This allows us to discuss the relationships among these various theories as well as the individual theories themselves. Roughly speaking we show that each of these theories is a "bundle" theory with a certain structure group. In the case of approximate fibrations, the groups are the simplicial groups $\operatorname{Top}^{c}\left(q_{0}\right)$, where $q_{0}: V \rightarrow \mathbf{R}^{i}$ is a manifold approximate fibration, and $T o p^{c}\left(q_{0}\right)$ denotes the simplicial group of controlled homeomorphisms of $q_{0}$ (these terms are defined in $\S 1$ ).

Suppose $p: E \rightarrow B$ is a fibration with fibre $F$. The problem of finding a manifold approximate fibration which is controlled homotopy equivalent to $p$ is equivalent to finding a manifold approximate fibration $q_{0}: V \rightarrow \mathbf{R}^{i}$ where $V$ is homotopy equivalent to $F$ and then showing that certain obstructions in $H^{*}\left(B ; \pi_{*-1}\left(G(F) / \operatorname{Top}^{c}\left(q_{0}\right)\right)\right)$ vanish (see $\left.\S 1 . \mathrm{E}\right)$.

Suppose $q: M \rightarrow B^{i}$ is a manifold approximate fibration and $q_{0}=q \mid q^{-1}(U)$ where $U \cong \mathbf{R}^{i}$ is an open subset of $B$. The problem of finding a fibre bundle with closed manifold fibre which is controlled homeomorphic to $q$ is equivalent to finding a closed manifold $P$ where $p_{2}: P \times \mathbf{R}^{i} \rightarrow \mathbf{R}^{i}$ is controlled homeomorphic to $q_{0}$ and then showing that certain obstructions in $H^{*}\left(B ; \pi_{*-1}\left(\operatorname{Top}^{c}\left(p_{2}\right) / \operatorname{Top}(P)\right)\right)$ vanish (see $\left.\S 1 . \mathrm{F}\right)$.

We would like to thank W. Dwyer for numerous invaluable conversations. In particular, the proof of Lemma 7.10 was worked out in conjunction with him.

\section{BACKGROUND AND STATEMENT OF RESULTS}

Let $B^{i}$ be a topological manifold of dimension $i$. First recall the classification of Hurewicz fibrations and fibre bundles over $B$.

1.A. Fibrations. Let $F$ be a locally-finite $\mathrm{CW}$ complex. There exists a bijection

$\left\{\begin{array}{c}\text { fibre homotopy equivalence classes of fibrations } \\ \text { over } B \text { with fibre homotopy equivalent to } F\end{array}\right\} \rightarrow[B, B G(F)]$,

$$
(p: E \rightarrow B) \rightarrow \hat{p}: B \rightarrow B G(F),
$$

where $G(F)$ denotes the simplicial monoid of homotopy automorphisms of $F$. We also use $B$ to denote the singular complex of $B$ so we may think of $[B, B G(F)]$ either as the set of simplicial homotopy classes of simplicial maps or as the set of ordinary homotopy classes of maps between the two geometric realizations.

Furthermore, for any fibration $p: E \rightarrow B$, there is a homotopy equivalence

$$
B G^{f}(E \rightarrow B) \rightarrow\left\{\begin{array}{c}
\text { path component of } M a p(B, B G(F)) \\
\text { which contains } \hat{p}: B \rightarrow B G(F)
\end{array}\right\}
$$

where $G^{f}(E \stackrel{p}{\rightarrow} B)$ denotes the simplicial monoid of fibre homotopy automorphisms of $p: E \rightarrow B$. (See Dror, Dwyer and Kan [10] for the Kan fibration analogue.) 
1.B. Fibre bundles. Suppose $F$ is a compact manifold (Hilbert cube or finite dimensional). There exists a bijection

$$
\left\{\begin{array}{c}
\text { fibre homeomorphism classes } \\
\text { of fibre bundles over } B \text { with } \\
\text { fibres homeomorphic to } F
\end{array}\right\} \longrightarrow[B, B T O P(F)],
$$

$$
(p: E \rightarrow B) \longrightarrow \hat{p}: B \rightarrow B T O P(F),
$$

Furthermore, for any fibre bundle $p: E \rightarrow B$ there is a homotopy equivalence

$$
B T O P^{f}(E \stackrel{p}{\rightarrow} B) \longrightarrow\left\{\begin{array}{c}
\text { path component of } \operatorname{Map}(B, B T O P(F)) \\
\text { which contains } \hat{p}: B \rightarrow B T O P(F)
\end{array}\right\}
$$

where $\operatorname{TOP}^{f}(E \stackrel{p}{\rightarrow} B)=$ the singular complex of the group $\{h \in T O P(E) \mid p=$ $p \circ h\}$.

Given a fibration with fibre homotopy equivalent to $F$, then there exists a fibre bundle $\tilde{p}: \tilde{E} \rightarrow B$ with fibre $F$ and a fibre homotopy equivalence $\tilde{E} \rightarrow E$ iff we can lift $\hat{p}: B \rightarrow B G(F)$ thru $B T O P(F) \rightarrow B G(F)$.

1.C. Approximate fibrations. Suppose that we have two spaces over $B, q_{j}: X_{j} \rightarrow$ $B, j=0,1$. A controlled map from $q_{0}$ to $q_{1}$ is given by a level-preserving map $F: X_{0} \times[0,1) \rightarrow X_{1} \times[0,1)$ such that the map $\left(q_{1} \times i d\right) \circ F \cup q_{0} \times 1: X_{0} \times[0,1] \rightarrow$ $B$ is continuous (see Definition 12.1). When we want to indicate that we have a controlled map from $q_{0}$ to $q_{1}$ we will write $F^{c}: X_{0} \rightarrow X_{1}$. We will write $F_{t}^{c}$ to denote the map induced by $F$ on $X_{0} \times t$.

Recall that the mapping cylinder of $q_{j}$, denoted $M\left(q_{j}\right)$, is the quotient space of $X_{j} \times[0,1] \Perp B$ in which we identify $x \times 1$ with $q_{j}(x)$. Notice that $M\left(q_{j}\right)=X_{j} \times[0,1) \cup B$. A level-preserving map between mapping cylinders $M\left(q_{0}\right) \rightarrow M\left(q_{1}\right)$ is a map $F_{t}$ which takes $X_{0} \times t$ into $X_{1} \times t$ for each $t$ in $[0,1)$. A level-preserving map $M\left(q_{0}\right) \rightarrow M\left(q_{1}\right)$ which is the identity on $B$ always induces a unique controlled map. The converse is true if $q_{1} \times$ id: $X_{1} \times[0,1] \rightarrow$ $B \times[0,1]$ is a closed map (see Lemma 12.2).

A second controlled map $H^{c}$ is controlled homotopic to $F^{c}$ if there exists a controlled map $G^{c}$ from $X_{0} \times[0,1] \stackrel{q_{0} \circ p_{1}}{\rightarrow} B$ to $X_{1} \times[0,1] \stackrel{q_{1} \circ p_{1}}{\rightarrow} B$ such that $G^{c} \mid X_{0} \times 0=F^{c}$ and $G^{c} \mid X_{1} \times 1=H^{c}$ (here $p_{1}$ denotes projection onto the first coordinate).

Any map $f: X_{0} \rightarrow X_{1}$ such that $q_{1} \circ f=q_{0}$ can be considered a controlled map by letting the family of maps be $f$ at each level. In particular, the identity map is naturally a controlled map. Hence we may define the notion of controlled homotopy automorphisms and controlled homeomorphism in the usual way.

A space over $B, q: X \rightarrow B$ is an approximate fibration if given any commutative diagram of continuous functions

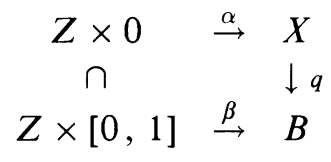


there exists a controlled map $f^{c}$ from $\beta: Z \times[0,1] \rightarrow B$ to $q: X \rightarrow B$ such that $f(z, 0, t)=\alpha(z)$ for all $z \in Z$ and $0 \leq t<1$.

Definition 1.1. Suppose $q: X \rightarrow B$ is an approximate fibration. We define the simplicial monoid of controlled homotopy equivalences of $q$, denoted $G^{c}(q)$ as follows: a $k$-simplex of $G^{c}(q)$ is given by a controlled map $h^{c}$ from $q \circ p_{1}: X \times$ $\Delta^{k} \rightarrow B$ to itself such that

(i) $h^{c}$ is a controlled homotopy equivalence, and

(ii) for each $0 \leq t<1$, the following diagram commutes

$$
\begin{array}{ccc}
X \times \Delta^{k} & \stackrel{h_{t}^{c}}{\rightarrow} & X \times \Delta^{k} \\
p_{2} \searrow & & \swarrow p_{2} \\
& \Delta^{k} &
\end{array}
$$

In the definition above, $p_{i}$ denotes projection onto the $i$ th factor in a product.

For any map $q: X \rightarrow B$, we let $E(q): E(X) \rightarrow B$ denote the associated Hurewicz fibration. To fix notation, $E(X)=\left\{(x, \lambda) \in X \times B^{I} \mid q(x)=\lambda(0)\right\}$ and $E(q)(x, \lambda)=\lambda(1)$. Our first result states that for many purposes an approximate fibration is equivalent to its associated Hurewicz fibration.

Theorem 1.2. Let $B$ be a separable metric space.

(i) We get a bijection

$\left\{\begin{array}{l}\text { controlled homotopy classes of } \\ \text { approximate fibrations over } B \\ \text { with separable metric total space }\end{array}\right\} \rightarrow\left\{\begin{array}{l}\text { fibre-homotopy classes } \\ \text { of fibrations over } B \text { with } \\ \text { separable metric total space }\end{array}\right\}$,

$$
(q: X \rightarrow B) \rightarrow(E(q): E(X) \rightarrow B) .
$$

(ii) For any fibration $q: X \rightarrow B$ with $X$ separable metric, we get a homotopy equivalence

$$
B G^{f}(q) \rightarrow B G^{c}(q)
$$

1.D. Manifold approximate fibrations. A map $q: M \rightarrow B$ is a manifold approximate fibration if $M$ is a manifold (Hilbert cube or finite dimensional); $q$ is a proper map which is an approximate fibration; and $\partial M=q^{-1}(\partial B)$. We let $\operatorname{TOP}^{c}(q)$ denote the simplicial group where a $k$-simplex is given by a controlled homeomorphism from $q \circ p_{1}: M \times \Delta^{k} \rightarrow B$ to itself which is fibre preserving over $\Delta^{k}$. Notice that $T O P^{c}(q)$ is a subsimplicial monoid of $G^{c}(q)$.

Suppose $q_{0}: V \rightarrow \mathbf{R}^{i}$ is a manifold approximate fibration. Then $\bar{q}_{0}$, the conjugate of $q_{0}$, is given by the composition

$$
V \stackrel{q_{0}}{\rightarrow} \mathbf{R}^{i}=\mathbf{R} \times \mathbf{R}^{i-1} \stackrel{(-1) \times \mathrm{id}}{\longrightarrow} \mathbf{R} \times \mathbf{R}^{i-1}=\mathbf{R}^{i} .
$$

If $q_{0}$ and $\bar{q}_{0}$ are controlled homeomorphic, then we say that $q_{0}$ is self-conjugate. Notice that if $V$ is homeomorphic to $X \times \mathbf{R}^{1}$ for any space $X$, then $q_{0}$ is self-conjugate. 
Definition 1.3. Suppose that $q: M \rightarrow B$ is a manifold approximate fibration and that $\alpha: \mathbf{R}^{i} \rightarrow B^{i}$ is an embedding. Then $q \mid: q^{-1}\left(\alpha\left(\mathbf{R}^{i}\right)\right) \rightarrow \mathbf{R}^{i}$ is a manifold approximate fibration (see Corollary 12.14) which we call the fibre germ of $q$ over $\alpha$. We say that $q$ has fibre germ $q_{0}$ iff there is a controlled homeomorphism between $q_{0}: V \rightarrow \mathbf{R}^{i}$ and $q \mid$.

If $B$ is path connected and $\operatorname{dim} M \geq 5$, then we will show in Corollary 14.6 that the fibre germ of $q$ over $\alpha$ is independent of $\alpha$ up to controlled homeomorphism and conjugacy. We also show there that, if $q$ has fibre germ $q_{0}$, and $q_{0}$ is not self-conjugate then $B$ is oriented by requiring that $\alpha$ have degree 1 .

Let $T O P_{i}$ denote the singular complex of the topological group of origin preserving homeomorphisms of $\mathbf{R}^{i}$ and let $S T O P_{i}$ denote the subgroup of orientation preserving elements in $T O P_{i}$.

Given a manifold approximate fibration, $q_{0}: V \rightarrow \mathbf{R}^{i}$, there is an associated bundle constructed in $\S 4, M A F(\mathscr{U}) \rightarrow B T O P_{i}$. If $q_{0}$ is self-conjugate, let $\eta: M A F\left(q_{0}\right) \rightarrow B T O P_{i}$ also denote this bundle. If $q_{0}$ is not self-conjugate, then the bundle map $M A F(\mathscr{U}) \rightarrow B T O P_{i}$ factors thru $B S T O P_{i}$ and we let $\eta: M A F\left(q_{0}\right) \rightarrow B S T O P_{i}$ denote this bundle.

Theorem 1.4 (Classification of manifold approximate fibrations). Suppose that $\operatorname{dim} V \geq 5$ and that $\partial B=\varnothing$. Given any manifold approximate fibration $q_{0}: V \rightarrow \mathbf{R}^{i}$, there exists a universal fibration

such that

$$
\eta: M A F\left(q_{0}\right) \rightarrow \begin{cases}B T O P_{i} & \text { if } q_{0} \text { is self-conjugate } \\ B S T O P_{i} & \text { if } q_{0} \text { is not self-conjugate }\end{cases}
$$

(a) the fibre of $\eta$ is $\operatorname{BTOP}^{c}\left(q_{0}\right)$

(b) if $q_{0}$ is self-conjugate we get a bijection

$\left\{\begin{array}{l}\text { controlled homeomorphism classes of } \\ \text { manifold approximate fibrations over } \\ B \text { with fibre germ } q_{0}\end{array}\right\} \rightarrow\left\{\begin{array}{l}\text { homotopy classes of } \\ \text { liftings of } B \stackrel{\hat{t}_{B}}{\rightarrow} B T O P_{i} \\ \text { thru } M A F\left(q_{0}\right)\end{array}\right\}$,

$$
(q: M \rightarrow B) \rightarrow\left(\hat{q}: B \rightarrow M A F\left(q_{0}\right)\right)
$$

with a similar diagram if $q_{0}$ is not self-conjugate except that the $B T O P_{i}$ is replaced by $B S T O P_{i}$ and where $\tau_{B}$ denotes the tangent bundle of $B$ and $\hat{\tau}_{B}$ denotes its classifying map into $B T O P_{i}$ if $q_{0}$ is self-conjugate or into $B S T O P_{i}$ if $q_{0}$ is not self-conjugate and we have oriented $B$

(c) for any manifold approximate fibration $q: M \rightarrow B$ with self-conjugate fibre germ $q_{0}: V \rightarrow \mathbf{R}^{i}$, we get a homotopy equivalence

$$
B \operatorname{BOP}^{c}(q) \longrightarrow\left\{\begin{array}{l}
\text { path component of } \\
\operatorname{Lift}\left(\begin{array}{cc} 
& \operatorname{MAF}\left(q_{0}\right) \\
& \downarrow \\
B \underset{\hat{t}}{\rightarrow} & B T O P_{i}
\end{array}\right) \\
\text { which contains } \hat{q}
\end{array}\right\}
$$


(if $q_{0}$ is not self-conjugate then we replace $B T O P_{i}$ by $B S T O P_{i}$ and the result still holds).

Example 1.5. Suppose that $B=S^{1}$. Then $q: M \rightarrow S^{1}$ is classified by a lift into $M A F\left(q_{0}\right)$ using 1.4(b). Since the tangent bundle of $S^{1}$ is trivial, this lift is given by a map

$$
\hat{q}: S^{1} \rightarrow B T O P^{c}\left(V \stackrel{q_{0}}{\rightarrow} \mathbf{R}^{1}\right) .
$$

Theorem $(1.4(\mathrm{c}))$ says that $B T O P^{c}(q)$ is homotopy equivalent to the space of unbased loops of $B T O P^{c}\left(V \stackrel{q_{0}}{\rightarrow} \mathbf{R}^{1}\right)$. For any simplicial set $\mathbf{X}$, the space $\Omega \operatorname{Map}\left(S^{1}, \mathbf{X}\right)$ is homotopy equivalent to $\Omega^{2} \mathbf{X} \times \Omega \mathbf{X}$. We then get

$\operatorname{TOP}^{c}(q)$ is homotopy equivalent to

$$
\Omega T O P^{c}\left(V \rightarrow \mathbf{R}^{1}\right) \times T O P^{c}\left(V \times \mathbf{R}^{1}\right) .
$$

If $V$ is homeomorphic to $N \times \mathbf{R}^{1}$ for some compact manifold $N$, then $q_{0}: V \rightarrow$ $\mathbf{R}^{1}$ is controlled homeomorphic to $p_{2}: N \times \mathbf{R}^{1} \rightarrow \mathbf{R}^{1}$;

$$
T O P^{c}\left(q_{0}\right) \cong T O P^{b}\left(N \times \mathbf{R}^{1}\right),
$$

the simplicial group of bounded homeomorphisms; and

$$
\Omega \operatorname{TOP}^{c}\left(q_{0}\right) \cong T O P(N \times[0,1] ; \text { rel } \partial) .
$$

(See Anderson-Hsiang [2] or Hsiang-Sharpe [15].)

Thus, in the special case where $q=p_{2}: N \times S^{1} \rightarrow S^{1}$, result (1.5.1) above and the above remarks yield

$$
\text { (1.5.2) } \operatorname{TOP}^{c}\left(N \times S^{1} \rightarrow S^{1}\right) \cong T O P(N \times[0,1], \text { rel } \partial) \times T O P^{b}\left(N \times \mathbf{R}^{1}\right) \text {. }
$$

Using the above equivalences and forgetting control, we get a map

$$
T O P(N \times[0,1], \text { rel } \partial) \times T O P^{b}\left(N \times \mathbf{R}^{1}\right) \rightarrow T O P\left(N \times S^{1}\right)
$$

which was originally constructed by Burghelea, Lashof and Rothenberg [3], [4].

In the case in which $N$ is a Hilbert cube manifold, the forgetting control map is the analogue in Waldhausen $K$-theory of the map in ordinary algebraic $K$-theory $K(\mathbb{Z} \pi) \times \Omega^{-1} K(\mathbb{Z} \pi) \rightarrow K(\mathbb{Z}[\pi \times \mathbb{Z}])$ which gives the Fundamental Theorem of algebraic $K$-theory.

The proof of (1.4) is based on showing that any manifold approximate fibration can be "fattened" into a fibre bundle.

Definition 1.6. Suppose $q: M \rightarrow B^{i}$ is a fibre bundle. Shrinking data for $q$ (if it exists) is given by a commutative diagram

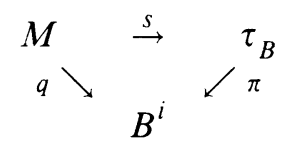

where, for each $b \in B, s: q^{-1}(b) \rightarrow \pi^{-1}(b) \cong \mathbf{R}^{i}$ is a manifold approximate fibration. 
Example. Suppose $M=M_{1} \times_{B} \tau_{B}$, where $M_{1} \rightarrow B$ is a fibre bundle with closed manifold fibres. Then the projection map $M \rightarrow \tau_{B}$ is shrinking data for the fibre bundle $M \rightarrow B$.

Definition 1.7. Suppose that we have two fibre bundles $q_{j}: M_{j} \rightarrow B, j=0,1$, with shrinking data $s_{j}: M_{j} \rightarrow \tau_{B}$. The two pairs $\left(q_{0}, s_{0}\right)$ and $\left(q_{1}, s_{1}\right)$ are concordant if there exists a fibre bundle over $B \times[0,1]$ with shrinking data that extends $q_{0} \Perp q_{1}$ and $s_{0} \Perp s_{1}$ over $(B \times 0) \Perp(B \times 1)$.

Our proof of (1.4) uses a parametrized version of the following result which should be of independent interest.

Theorem 1.8. There exists a bijection

$\left\{\begin{array}{l}\text { controlled homeomorphism classes } \\ \text { of manifold approximate fibrations } \\ \text { over } B\end{array}\right\} \rightarrow\left\{\begin{array}{l}\text { concordance classes of fibre } \\ \text { bundles with shrinking data } \\ \text { over } B\end{array}\right\}$.

1.E. Making fibrations into manifold approximate fibrations. Assume that $B^{i}$ is a topological manifold of dimension $i, \partial B=\varnothing$, and that $F$ is a locally-finite $\mathrm{CW}$ complex. Suppose that $q_{0}: V \rightarrow \mathbf{R}^{i}$ is a manifold approximate fibration, where $V$ is homotopy equivalent to $F$. If $q_{0}$ is self-conjugate, we get a commutative diagram

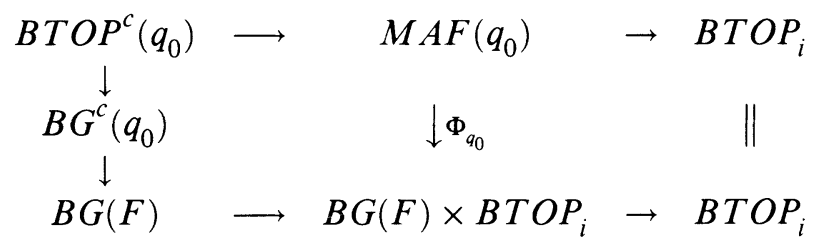

where the top row is the fibration from (1.4); the bottom row is the trivial fibration which comes from our proof of the classification of fibrations; and the vertical maps come from the naturality of our constructions. If $q_{0}$ is not selfconjugate there is a similar diagram with the $B T O P_{i}$ 's replaced by $B S T O P_{i}$ 's.

Theorem 1.9. Suppose that $p: E \rightarrow B^{i}$ is a fibration with fibre $F$. Then, $p$ is controlled homotopy equivalent to a manifold approximate fibration with selfconjugate fibre germ $q_{0}: V \rightarrow \mathbf{R}^{i}$ iff we can solve the following lifting problem

$$
\begin{aligned}
& \operatorname{MAF}\left(q_{0}\right) \\
& \downarrow \Phi_{q_{0}} \\
& B \stackrel{\hat{p} \times \hat{\tau}_{B}}{\rightarrow} \quad B G(F) \times B T O P_{i}
\end{aligned}
$$

There is a similar result for non-self-conjugate fibre germs with $B T O P_{i}$ replaced by BSTOP ${ }_{i}$. Notice that the fibre of $\Phi_{q_{0}}$ is homotopy equivalent to $G\left(q_{0}\right) / \operatorname{TOP}^{c}\left(q_{0}\right)$, which is the same as $G^{c}\left(q_{0}\right) / T O P^{c}\left(q_{0}\right)$ by Proposition 9.2.

Corollary 1.10. Suppose that $F \times T^{i}$ is homotopy equivalent to a closed, compact manifold of dimension $\geq 5$. Then, for any parallelizable manifold $B^{i}$ with $\partial B=\varnothing, F \times B^{i}$ is homotopy equivalent to a manifold. 
Remark. From this and the $\pi-\pi$ Theorem of Wall [40] it follows that if $B^{i}$ is oriented bordant over its fundamental group to a parallelizable manifold, $F \times B^{i}$ is homotopy equivalent to a manifold.

Proof. The Kirby torus trick [26] converts the homotopy manifold structure on $F \times T^{i}$ into a bounded, and hence controlled (see [24] or the proof of Proposition 3.2 in [19] plus $\S 13$ of this paper), homotopy structure on $F \times \mathbf{R}^{i}$. Then $\hat{p} \times \hat{\tau}$ is trivial in (1.9) so we can certainly solve the required lifting problem.

At the start of $\S 11$, we give the definition of the controlled structure set for any fibration $q: E \rightarrow B$, where $B$ is a manifold with $\partial B=\varnothing$. We denote this simplicial set by $\mathscr{S}(q: E \rightarrow B)$. Roughly, a $k$-simplex is a manifold approximate fibration $M \rightarrow B \times \Delta^{k}$ (which is a fibre bundle over $\Delta^{k}$ ) together with a fibre map $f: M \rightarrow E \times \Delta^{k}$ which is a controlled homotopy equivalence (fibred over $\left.\Delta^{k}\right)$. This extends the definition given in [22].

Theorem 1.11. Let $q: E \rightarrow B^{i}$ be a fibration with closed Poincaré space fibre of dimension $n$. Then, if $n+i \geq 5$,

$$
\mathscr{S}(q: E \rightarrow B)=\coprod G^{c}(q) / \operatorname{Top}^{c}(\tilde{q})
$$

where, on the right, we take the union over all homeomorphism classes of manifold approximate fibrations $\tilde{q}: M^{n+i} \rightarrow B^{i}$ which are controlled homotopy equivalent to $q$ (if $n=\infty$ then $M$ is a Hilbert cube manifold).

Remarks. Just as in the uncontrolled case, there is no natural map of monoids $T o p^{c}(\tilde{q}) \rightarrow G^{c}(q)$ for which $G^{c}(q) / \operatorname{Top}^{c}(\tilde{q})$ is the quotient. In $\S 9$ we describe a map $B T o p^{c}(\tilde{q}) \rightarrow B G^{c}(q)$, and its homotopy fibre is what we mean by $G^{c}(q) / \operatorname{Top}^{c}(\tilde{q})$.

The reader might have expected us to define the controlled structure set a bit differently. An equally attractive definition would be to replace the fibre map $f$ in the above definition by a controlled map $f^{c}$. We show in Theorem 11.1 that we get homotopy equivalent simplicial sets. The monoid $G^{c}(q)$ acts naturally on this last definition, and it is a corollary of Proposition 8.2 that this action is equivalent to the homotopy action coming from the definition of $G^{c}(q) / \operatorname{Top}^{c}(\tilde{q})$ as a homotopy fibre.

Consider the map

$$
\Upsilon_{n}: \coprod M A F(\mathscr{U}) \rightarrow B G(F) \times B T O P_{i}
$$

where we take the disjoint union over all the homeomorphism classes of manifold approximate fibrations $q_{0}: V^{n+i} \rightarrow \mathbf{R}^{i}$ with $V$ homotopy equivalent to $F$. Notice that the fibre of $\Upsilon_{n}$ is $\mathscr{S}\left(p_{2}: F \times \mathbf{R}^{i} \rightarrow \mathbf{R}^{i}\right)$. 
Theorem 1.12. For any fibration $q: X \rightarrow B^{i}$ with $n+i \geq 5$, there exists a homotopy equivalence

$$
\mathscr{S}(q: X \rightarrow B) \rightarrow \operatorname{Lift}\left(\begin{array}{c}
M A F(\mathscr{U}) \\
\downarrow \Upsilon_{n} \\
B \rightarrow B G(F) \times B T O P_{i}
\end{array}\right)
$$

where $F$ is the homotopy fibre of the map $q$.

Remark. This is an improvement of the main result in [22] in that it applies in more general situations and in that it gives existence of structures iff there exist lifts.

In order to apply (1.12) we need results about $\mathscr{S}\left(p_{2}: F \times \mathbf{R}^{i} \rightarrow \mathbf{R}^{i}\right)$ where $F$ is a compact Hilbert cube manifold. Results of Chapman [5] and Hughes [18] imply that for $j \leq i$,

$\pi_{j}\left(\mathscr{S}\left(p_{2}: F \times \mathbf{R}^{i} \rightarrow \mathbf{R}^{i}\right)\right) \cong W h_{j-i+1}\left(\pi_{1}(F)\right)= \begin{cases}W h_{1}\left(\pi_{1} F\right) & \text { if } j-i+1=1, \\ \tilde{K}_{0}\left(\mathbb{Z} \pi_{1} F\right) & \text { if } j-i+1=0 \\ K_{j-i+1}\left(\mathbb{Z} \pi_{1} F\right) & \text { if } j-i+1<0\end{cases}$

Corollary 1.13. Suppose that $p: E \rightarrow B^{i}$ is a fibration with fibre $F$ such that $F$ is dominated by a finite $C W$ complex. If $W h_{k}\left(\pi_{1} F\right) \cong 0$ for $k \leq 0$, then there exists a $Q$-manifold approximate fibration which is controlled homotopy equivalent to $p: E \rightarrow B$. If we further assume that $W h_{1}\left(\pi_{1} F\right) \cong 0$, then the resulting $Q$-manifold approximate fibration is unique up to controlled homeomorphism.

One natural way to try to study the controlled structure set would be to use some sort of controlled surgery theory. We intend to do this in a sequel [24].

1.F. Making approximate fibrations into fibre bundles. Assume that $B^{i}$ is a topological manifold of dimension $i, \partial B=\varnothing$. Suppose that $N$ is a compact space such that $N \times \mathbf{R}^{i}$ is a manifold without boundary of dimension $n+i \geq$ 5. Then $p_{2}: N \times \mathbf{R}^{i} \rightarrow \mathbf{R}^{i}$ is a manifold approximate fibration, and we get a commutative diagram

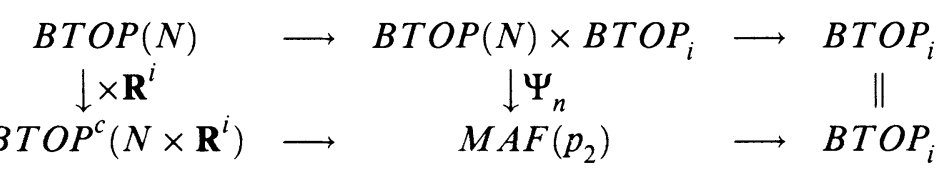

where the bottom row is the fibration from (1.4). The fibre here is clearly self-conjugate.

Theorem 1.14. Suppose that $p: M \rightarrow B^{i}$ is a manifold approximate fibration with fibre germ controlled homeomorphic to $p_{2}: N \times \mathbf{R}^{i} \rightarrow \mathbf{R}^{i}$. Then, $p$ is controlled homeomorphic to a fibre bundle with fibre homeomorphic to $N$ iff we can solve the following lifting problem:

$$
\begin{array}{ccc} 
& \operatorname{BTOP}(N) \times \text { BTOP }_{i} \\
& & \downarrow \Psi_{n} \\
B^{i} & \stackrel{\hat{p}}{\rightarrow} & M A F\left(p_{2}\right)
\end{array}
$$


Notice that the fibre of $\Psi_{n}$ is homotopy equivalent to $\operatorname{TOP}^{c}\left(N \times \mathbf{R}^{i}\right) / T O P(N)$.

The following result is proved in [41] (for the equivalence of bounded and controlled homeomorphisms see [23]).

Theorem 1.15. If $N$ is a compact, closed, finite dimensional manifold then $\operatorname{TOP}^{c}\left(N \times \mathbf{R}^{i}\right) / T O P(N)$ is homotopy equivalent to $Q\left(S_{+}^{i-1} \wedge_{\mathbb{Z} / 2} \mathscr{S}(N \times Q)_{0}\right)$ through the concordance stable range for $N$, where $\mathscr{S}(N \times Q)_{0}$ denotes the component of $\mathscr{S}(N \times Q)$ containing the identity.

In the case where $N$ is a Hilbert cube manifold one also has the following results.

Theorem 1.16. If $N$ is a compact Hilbert cube manifold, then, for any $i \geq 0$, the map

$$
\mathscr{S}\left(p_{2}: N \times \mathbf{R}^{i} \rightarrow \mathbf{R}^{i}\right) \stackrel{\times \mathbf{R}^{1}}{\rightarrow} \mathscr{S}\left(p_{2}: N \times \mathbf{R}^{i+1} \rightarrow \mathbf{R}^{i+1}\right)
$$

is homotopy trivial.

Corollary 1.17. If $N$ is a compact Hilbert cube manifold and $i>0$, then $T O P^{c}\left(N \times \mathbf{R}^{i}\right) / T O P(N)$ is homotopy equivalent to the union of certain components of the homotopy fibre of the map $\mathscr{S}(N) \rightarrow \mathscr{S}\left(p_{2}: N \times \mathbf{R}^{i+1} \rightarrow \mathbf{R}^{i+1}\right)$, which is just $\mathscr{S}(N) \times \Omega \mathscr{S}\left(p_{2}: N \times \mathbf{R}^{i+1} \rightarrow \mathbf{R}^{i+1}\right)$.

1.G. Outline of proofs. In this section we give a brief guide to the overall strategy of the proofs and indicate how the results in the introduction follow from the results proved in the remainder of this paper. Our first step is to introduce simplicial sets $B u n(B), M A F(B), H u r(B)$. Roughly speaking these are simplicial sets where the $k$-simplices are maps $E \rightarrow B \times \Delta^{k}$ which are bundles, manifold approximate fibrations, Hurewicz fibrations respectively. The actual definitions are given in $\S 2$ and involve a few technical wrinkles, but the above is basically correct.

The bulk of this paper is devoted to proving two sorts of theorems. In $\S \S 2-$ 4, we prove that our simplicial sets can be described as spaces of lifts or as spaces of maps. These results culminate in Examples 4.8 and 4.9. In $\S \S 5-7$ we prove that path components of our simplicial sets are homotopy equivalent to the classifying space of the appropriate simplicial monoids. These results are summarized in Corollaries 7.12, 7.13, 7.14 and 7.15. From these results one can easily deduce the results in 1.A on Hurewicz fibrations. $\S 9$ shows that controlled homotopy equivalences are sufficiently like fibre homotopy equivalences that the results in $\S 1$.A on approximate fibrations also follow. Moreover, the same strategy also shows the results in $\S \S 1 . B$ and 1.D.

The material on shrinking data is introduced along the way in $\S \S 2$ and 3. The simplicial sets of shrinking data arise as an intermediate step in the proof that the simplicial set of manifold approximate fibrations is a space of lifts, and they seemed sufficiently interesting in their own right to warrant treatment in the introduction. 
Finally, we have taken some trouble to derive our results for these various simplicial sets in a uniform manner, so that it is easy to compare the different classifications. The material in 1.E and 1.F follows as indicated there by comparing the different classifications.

\section{Simplicial SETS AND SIMPlicial CATEGoRies OF BUNDLES, APPROXIMATE FIBRATIONS AND FIBRATIONS}

Let $l_{2}$ denote the usual Hilbert space of sequences of real numbers $\left(x_{0}, x_{1}\right.$, $\ldots)$ such that $\sum_{r=0}^{\infty} x_{r}^{2}<\infty$. Let $\nu_{i}\left(x_{0}, \ldots, x_{i}, \ldots\right)=x_{i}$. We say that a subset, $X$, of $l_{2}$ has small capacity provided there exist integers $r$ and $m$ such that $\nu_{i}(x)=0$ for all $i \equiv r(\bmod m)$ and all $x \in X$. We say that a subset, $X$, of $l_{2} \times Y$ has small capacity provided that the image of $X$ in $l_{2}$ under the projection has small capacity.

Define a countable collection of points, $v_{i}, i=0,1, \ldots$, by $\nu_{i}\left(v_{j}\right)=\delta_{i, j+1}$. We let $\Delta^{k}$ denote the convex set spanned by $\left\{v_{0}, \ldots, v_{k}\right\}$ (which of course is a $k$-simplex). Finally, we will have occasion below to mention $\Delta^{k} \times[0,1]$. This is just the subset of $l_{2}$ consisting of points whose 0 th coordinate lies between 0 and 1 , and whose other coordinates correspond to a point in $\Delta^{k}$.

Let $B$ be a topological $i$-dimensional manifold without boundary. In this section we define various simplicial sets which consist of bundles, approximate fibrations or fibrations over $B$ and discuss a differential from these sets to various simplicial sets of lifts. Let $n \geq 0$ be a fixed integer such that $i+n \geq 5$. Fix a topological tangent bundle for $B, p_{1}: T B \rightarrow B$, where $T B \subset B \times B$ is a neighborhood of the diagonal and $p_{1}$ is the projection onto the first coordinate.

2.1 Manifold bundles over $B$. A $k$-simplex of the simplicial set $B u n(B)$ consists of a subset $M$ of $l_{2} \times B \times \Delta^{k}$ of small capacity such that

(i) the projection $p: M \rightarrow B \times \Delta^{k}$ is a fibre bundle projection with compact fibres, and

(ii) the fibres of the composition $M \stackrel{p}{\rightarrow} B \times \Delta^{k} \rightarrow \Delta^{k}$ are $(n+i)$-dimensional manifolds without boundary.

A $k$-simplex of the simplicial set $\overline{B u n}(B)$ consists of a subset $M$ of $l_{2} \times$ $T B \times \Delta^{k}$ of small capacity such that the following hold.

(i) the projection $p: M \rightarrow T B \times \Delta^{k}$ is a fibre bundle projection with compact fibres, and

(ii) the fibres of the composition $M \stackrel{p}{\rightarrow} T B \times \Delta^{k} \rightarrow \Delta^{k}$ are $(n+2 i)$ dimensional manifolds without boundary.

Boundary and face operations on $B u n(B)$ and $\overline{B u n}(B)$ are induced from those on $\Delta^{k}$ in the obvious way.

Define a simplicial map $d: B u n(B) \rightarrow \overline{B u n}(B)$, called the differential, as follows. If $M \subset l_{2} \times \Delta^{k}$ is a $k$-simplex of $\operatorname{Bun}(B)$, then

$$
d(M)=\left\{\left(x, b_{1}, b_{2}, y\right) \in l_{2} \times T B \times \Delta^{k} \mid\left(x, b_{2}, y\right) \in M\right\} .
$$


Proposition 2.1. The differential $d: B u n(B) \rightarrow \overline{B u n}(B)$ is a homotopy equivalence.

Proof. There is a map $f: \overline{B u n}(B) \rightarrow B u n(B)$ which takes a bundle over $T B \times \Delta^{k}$ and restricts it over $\Delta B \times \Delta^{k}$ (where $\Delta B$ is the diagonal). Clearly, $f \circ d=\mathrm{id}$. It is not too hard to construct a homotopy from $d \circ f$ to the identity.

2.2 Manifold approximate fibrations over $B$. A $k$-simplex of the simplicial set $M A F(B)$ consists of a subset $M$ of $l_{2} \times B \times \Delta^{k}$ of small capacity such that

(i) the projection $M \rightarrow \Delta^{k}$ is a fibre bundle projection with fibres $(n+i)$ dimensional manifolds without boundary, and

(ii) the projection $p: M \rightarrow B \times \Delta^{k}$ has the property that for each $x \in \Delta^{k}$, $p \mid: p^{-1}(B \times x) \rightarrow B \times x$ is a manifold approximate fibration.

A $k$-simplex of the simplicial set $\overline{M A F}(B)$ consists of a subset $M$ of $l_{2} \times$ $T B \times \Delta^{k}$ of small capacity such that

(i) the composite $M \stackrel{\text { proj }}{\rightarrow} T B \times \Delta^{k} \stackrel{p_{1} \times \text { id }}{\rightarrow} B \times \Delta^{k}$ is a fibre bundle projection with fibres $(n+2 i)$-dimensional manifolds without boundary, and

(ii) the projection $p: M \rightarrow T B \times \Delta^{k}$ has the property that for each $(x, y) \in$ $B \times \Delta^{k}, p \mid: p^{-1}\left(p_{1}^{-1}(x) \times y\right) \rightarrow p_{1}^{-1}(x) \times y$ is a manifold approximate fibration.

As in the bundle case there is a differential $d: M A F(B) \rightarrow \overline{M A F}(B)$, but in this case it takes a bit of work to see that $d$ actually takes values in $\overline{M A F}(B)$.

Lemma 2.2.1. If $M \subset l_{2} \times B \times \Delta^{k}$ is a $k$-simplex of $M A F(B)$, then $d(M)$ is a $k$-simplex of $\overline{M A F}(B)$.

Proof. Siebenmann's Technical Bundle Theorem [27] (Theorem 1.1, p. 60) implies that $d(M) \rightarrow B \times \Delta^{k}$ is a bundle projection (see [22, Lemmas 4.1, 8.1]). The projection $d(M) \rightarrow T B \times \Delta^{k}$ is a family of approximate fibrations because each slice is the restriction of $M \rightarrow B \times \Delta^{k}$ over (an open subset of $B$ ) $\times \Delta^{k}$ (see Corollary 12.13).

The following theorem is the main result of $\S 3$.

Theorem 2.2.2. The differential $d: M A F(B) \rightarrow \overline{M A F}(B)$ is a homotopy equivalence.

2.3 Fibrations over $B$. A $k$-simplex of the simplicial set $\operatorname{Hur}(B)$ consists of a subset of $l_{2} \times B \times \Delta^{k}$ of small capacity such that the projection $p: M \rightarrow B \times \Delta^{k}$ is an Hurewicz fibration.

A $k$-simplex of the simplicial set $\overline{H u r}(B)$ consists of a subset of $l_{2} \times T B \times \Delta^{k}$ of small capacity such that the projection $p: M \rightarrow T B \times \Delta^{k}$ is an Hurewicz fibration.

As above, there is a differential $d: H u r(B) \rightarrow \overline{H u r}(B)$ and as in the bundle case the following proposition is not hard to prove. 
Proposition 2.3.1. The differential $d: H u r(B) \rightarrow \overline{H u r}(B)$ is a homotopy equivalence.

\section{THE DIFFERENTIAL $d: M A F(B) \rightarrow \overline{M A F}(B)$}

In this section we extend the definition of $M A F$ and $\overline{M A F}$ to certain subsets of $B$ and use this "sheafification" to prove that $d: M A F(B) \rightarrow \overline{M A F}(B)$ is a homotopy equivalence.

We first define the simplicial set $M A F(U)$ where $U$ is the interior of a compact codimension 0 submanifold of $B$. A $k$-simplex of $M A F(U)$ is given by a subset $M$ of $l_{2} \times U \times \Delta^{k}$ of small capacity where

(i) $p: M \rightarrow U \times \Delta^{k}$ is an approximate fibration and is the restriction to $M$ of the projection $l_{2} \times U \times \Delta^{k} \rightarrow U \times \Delta^{k}$

(ii) the composition $M \stackrel{p}{\rightarrow} U \times \Delta^{k} \rightarrow \Delta^{k}$ is a fibre bundle projection whose fibre is an $n$-manifold without boundary.

If $U_{1} \subset U_{2}$ are both interiors of compact codimension 0 submanifolds of $B$, there is a restriction map $r: M A F\left(U_{2}\right) \rightarrow M A F\left(U_{1}\right)$ defined as follows: if $p: M \rightarrow U_{2} \times \Delta^{k}$ is a $k$-simplex of $M A F\left(U_{2}\right)$, then $r(p)$ is $p \mid: p^{-1}\left(U_{1} \times \Delta^{k}\right) \rightarrow$ $U_{1} \times \Delta^{k}$. Another application of Siebenmann's Technical Bundle Theorem shows that the composition $p^{-1}\left(U_{1} \times \Delta^{k}\right) \rightarrow U_{1} \times \Delta^{k} \rightarrow \Delta^{k}$ is a bundle projection (see [17, Lemma 4.1]). This is the reason that we restrict attention to the interiors of compact submanifolds rather than to all open subsets of $B$.

The next step is to define $\overline{M A F}$ over open subsets of $B$. However, in order to get a differential and to have restriction maps, it turns out to be necessary to pass to germs over neighborhoods of the diagonal in $U \times U$. The resulting simplicial set is denoted by $\overline{G M A F}(U)$. The precise definition follows.

If $U$ is any open subset of $B$, then a $k$-simplex of $\overline{G M A F}(U)$ is an equivalence class $[f]$ represented by a diagram

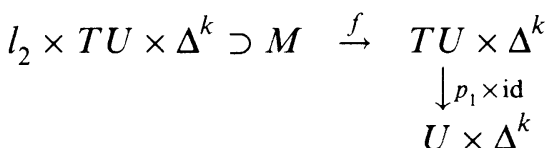

where:

(i) $T U$ is a tangent bundle neighborhood about the diagonal $\Delta U$ in $U \times B$ with $p_{1}: T U \rightarrow U$ the projection onto the first factor,

(ii) $p=\left(p_{1} \times\right.$ id $) \circ f$ is a fibre bundle projection whose fibre is an $(n+i)$ manifold without boundary,

(iii) $f$ is an approximate fibration,

(iv) $f$ is the restriction of the projection $l_{2} \times T U \times \Delta^{k} \rightarrow T U \times \Delta^{k}$

(v) $M$ is a subset of small capacity . 
Another such diagram

$$
\begin{aligned}
& l_{2} \times T^{\prime} U \times \Delta^{k} \supset M \stackrel{f^{\prime}}{\rightarrow} \quad T^{\prime} U \times \Delta^{k} \\
& \downarrow p_{1} \times \text { id } \\
& U \times \Delta^{k}
\end{aligned}
$$

is equivalent to $f$ provided that there is an open neighborhood $W$ of $\Delta U$ in $U \times B$ such that $W \subset T U \cap T^{\prime} U$ and $f=f^{\prime}$ over $W \times \Delta^{k}$.

Lemma 3.1. The simplicial sets $\overline{M A F}(B)$ and $\overline{G M A F}(B)$ are Kan.

Proof. We extend these simplicial sets to simplicial categories and prove the result as a corollary of Lemma 7.2. Of course the reader can verify this directly.

Proposition 3.2. The quotient map $\gamma: \overline{M A F}(B) \rightarrow \overline{G M A F}(B)$ is a homotopy equivalence.

Proof. We will show that $\gamma$ induces an isomorphism on homotopy groups. Suppose that $k \geq 0$ and that we are given a $k$-simplex [f] of $\overline{G M A F}(B)$ represented by $f: M \rightarrow T^{\prime} B \times \Delta^{k}$ where $T^{\prime} B$ is some tangent bundle neighborhood. By passing to a smaller neighborhood, we can assume that $T^{\prime} B \subset T B$, the fixed tangent bundle neighborhood. Suppose that we are additionally given a union of $k+1(k-1)$-simplices of $\overline{M A F}(B)$ described by a diagram

$$
l_{2} \times T B \times \partial \Delta^{k} \supset N \stackrel{g}{\rightarrow} \quad \begin{gathered}
T B \times \partial \Delta^{k} \\
\downarrow p_{1} \times \text { id } \\
B \times \partial \Delta^{k}
\end{gathered}
$$

such that $\gamma(g)=\partial([f])$. We need to show that there is a $k$-simplex $\tilde{g}$ in $\overline{M A F}(B)$ such that $\partial \tilde{g}=g$ and $\gamma(\tilde{g}) \cong[f]$ rel $\partial$.

By the Kister-Mazur Theorem there is a bundle isomorphism $j: T^{\prime} B \rightarrow T B$ which is the identity on some neighborhood of the diagonal. Then the composition

$$
\tilde{f}: M \stackrel{f}{\rightarrow} T^{\prime} B \times \Delta^{k} \stackrel{j \times \text { id }}{\rightarrow} T B \times \Delta^{k}
$$

describes a $k$-simplex of $\overline{M A F}(B)$ such that $\gamma(\tilde{f})=[f]$. Now $\tilde{g}$ is easily constructed using $\S 14$ to build a collar between $\partial \tilde{f}$ and $g$.

For $V \subset U$ we have a restriction $r: \overline{G M A F}(U) \rightarrow \overline{G M A F}(V)$.

We now define the differential $d: M A F(U) \rightarrow \overline{G M A F}(U)$ whenever $U$ is an open subset of $B$. Let $l_{2} \times U \times \Delta^{k} \supset M \stackrel{p}{\rightarrow} U \times \Delta^{k}$ describe the $k$-simplex $p$ of $M A F(U)$. Consider the map id $\times p: U \times M \rightarrow U \times U \times \Delta^{k}$ and let $T U \subset U \times U$ be a tangent bundle neighborhood. Let $\bar{M}=\left(\mathrm{id} \times p^{-1}\right)\left(T U \times \Delta^{k}\right)$ and $f=(\operatorname{id} \times p) \mid \bar{M}: \bar{M} \rightarrow T U \times \Delta^{k}$. Now $\bar{M} \subset U \times M \subset U \times l_{2} \times U \times \Delta^{k}$, but we rewrite this (by interchanging the first two factors) as $\bar{M} \subset l_{2} \times U \times U \times \Delta^{k}$. Then we have the diagram

$$
l_{2} \times U \times B \times \Delta^{k} \supset \bar{M} \stackrel{f}{\rightarrow} \quad T U \times \Delta^{k}
$$


It follows from Corollary 12.14 that $f$ is an approximate fibration and $p=$ $\left(p_{1} \times\right.$ id $) \circ f$ is a bundle projection. Thus $f$ represents a $k$-simplex $[f]$ in $\overline{G M A F}(U)$ and we define $d(p)=[f]$.

Note that whenever $V \subset U \subset B$ and $V$ and $U$ are the interiors of compact codimension 0 submanifolds of $B$, then the following diagram commutes

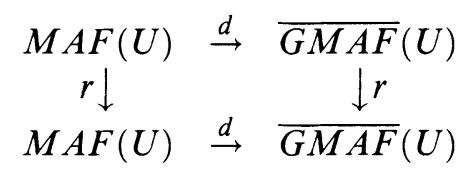

By passing to direct limits we define $M A F(X)$ and $\overline{G M A F}(X)$ for any compact subset $X \subset B$. The differential $d: M A F(X) \rightarrow \overline{G M A F}(X)$ is also defined.

The next step is to prove that the differential is a homotopy equivalence by applying Gromov's immersion theoretic machine as delineated by Siebenmann in [27, Essay V]. Thus, we need to verify certain properties of the restriction maps for $M A F$ and $\overline{M A F}$ and show that $d: M A F(x) \rightarrow \overline{G M A F}(x)$ is a homotopy equivalence for each point $x \in B$.

Proposition 3.3. If $X$ and $Y$ are compact subsets of $B$, then the following diagrams of restriction maps are fibre products (i.e. pull-back diagrams):
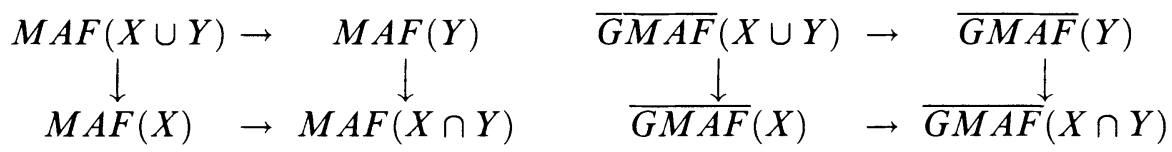

Proof. Suppose that $\tilde{X}$ and $\tilde{Y}$ are open neighborhoods, in $B$, of $X$ and $Y$ respectively, and suppose that $l_{2} \times \tilde{X} \times \Delta^{k} \supset M_{1} \stackrel{p_{1}}{\rightarrow} \tilde{X} \times \Delta^{k}$ and $l_{2} \times \tilde{Y} \times$ $\Delta^{k} \supset M_{2} \stackrel{p_{2}}{\rightarrow} \tilde{Y} \times \Delta^{k}$ represent $k$-simplices in $M A F(X)$ and $M A F(Y)$ such that $p_{1}=p_{2}$ over $(\tilde{X} \cap \tilde{Y}) \times \Delta^{k}$. Since $p_{1}$ and $p_{2}$ are the restrictions of projections, it follows that $M_{1} \cap M_{2}=p_{i}^{-1}(\tilde{X} \cap \tilde{Y}) \times \Delta^{k}$ for $i=1,2$. Let $M=M_{1} \cup M_{2}$ and let $p: M \rightarrow(\tilde{X} \cup \tilde{Y}) \times \Delta^{k}$ be defined by $p \mid M_{i}=p_{i}$ for $i=1,2$. Then $p$ is an approximate fibration (see [7]) and $l_{2} \times(\tilde{X} \cup \tilde{Y}) \times \Delta^{k} \supset$ $M \stackrel{p}{\rightarrow}(\tilde{X} \cup \tilde{Y}) \times \Delta^{k}$ represents a $k$-simplex in $M A F(X \cup Y)$. Since uniqueness is clear, this construction shows that the first diagram above is a fibre product.

An analogous argument works for $\overline{G M A F}$.

Proposition 3.4. If $Y \subset X$ are compact subsets of $B$, then the restrictions $r: M A F(X) \rightarrow M A F(Y)$ and $r: \overline{G M A F}(X) \rightarrow \overline{G M A F}(Y)$ are Kan fibrations.

Proof. We first show that $r: M A F(X) \rightarrow M A F(Y)$ is a Kan fibration. To this end, let $\alpha$ be a $(k+1)$-simplex of $M A F(Y)$ which is represented by a $(k+1)$-simplex of $M A F(V)$ described by

$$
l_{2} \times V \times[0,1] \supset N \stackrel{q}{\rightarrow} V \times \Delta^{k} \times[0,1]
$$

where $Y \subset V$ and $V$ is the interior of a compact codimension 0 submanifold of $B$ and we have identified $\Delta^{k+1}$ with $\Delta^{k} \times[0,1]$. 
Suppose that we are given a lift of the 0 -level of $\alpha$ to a $k$-simplex $\beta$ of $M A F(X)$. Thus $\beta$ is represented by a $k$-simplex of $M A F(U)$ described by

$$
l_{2} \times U \times \Delta^{k} \supset M \stackrel{p}{\rightarrow} U \times \Delta^{k}
$$

where $X \subset U$ and $U$ is the interior of a compact codimension 0 submanifold of $B$. By passing to a smaller neighborhood of $Y$ we can assume that $V \subset U$ and that $q=p$ over $V \times \Delta^{k} \times\{0\}$.

Let $h: M^{\prime} \times \Delta^{k} \rightarrow M$ be a trivializing homeomorphism for the bundle $M \rightarrow$ $\Delta^{k}$ where $M^{\prime}=M \cap\left(l_{2} \times U \times\left\{v_{0}\right\}\right)$ and $v_{0}$ is the 0 vertex of $\Delta^{k}$. Let $N^{\prime}=N \cap\left(l_{2} \times V \times\left\{v_{0}\right\} \times\{0\}\right)$ and choose a trivializing homeomorphism $g: N^{\prime} \times \Delta^{k} \times[0,1] \rightarrow N$ for the bundle $N \rightarrow \Delta^{k} \times[0,1]$ such that $g \mid N^{\prime} \times \Delta^{k} \times$ $\{0\}=h \mid$. Consider the compositions

$$
p^{\prime}: M^{\prime} \times \Delta^{k} \stackrel{h}{\rightarrow} U \times \Delta^{k} \text { and } q^{\prime}: N^{\prime} \times \Delta^{k} \times[0,1] \stackrel{g}{\rightarrow} N \stackrel{q}{\rightarrow} V \times \Delta^{k} \times[0,1] .
$$

Use the homotopy extension property for manifold approximate fibrations $(\S 14)$ to get an approximate fibration $\tilde{p}: M^{\prime} \times \Delta^{k} \times[0,1] \rightarrow U \times \Delta^{k} \times[0,1]$ such that $\tilde{p}$ is fibre preserving over $\Delta^{k} \times[0,1] ; \tilde{p}_{0}=p^{\prime}$; and $\tilde{p}=q^{\prime}$ over $V^{\prime} \times \Delta^{k} \times[0,1]$, where $V^{\prime} \subset V$ is an open neighborhood of $Y$.

Let $A=M^{\prime} \times \Delta^{k} \times\{0\} \cup \tilde{p}^{-1}\left(V^{\prime} \times \Delta^{k} \times[0,1]\right)$ and note that the following diagram commutes

$$
\begin{array}{ccc}
\stackrel{i}{A} & l_{2} \times U \times \Delta^{k} \times[0,1] \\
U \times \Delta^{k} \times[0,1] & = & U \times \Delta^{k} \times[0,1]
\end{array}
$$

where $i \mid M^{\prime} \times \Delta^{k} \times\{0\}=h^{-1}$ and $i \mid \tilde{p}^{-1}\left(V^{\prime} \times \Delta^{k} \times[0,1]\right)=g^{-1}$. Also $i: A \rightarrow l_{2} \times U \times \Delta^{k} \times[0,1]$ is a closed embedding of small capacity. It follows from elementary arguments that there is a closed embedding of small capacity $j: M^{\prime} \times \Delta^{k} \times[0,1] \rightarrow l_{2} \times U \times \Delta^{k} \times[0,1]$, extending $i$, which makes the following diagram commute

$$
\begin{array}{ccc}
M^{\prime} \times \Delta^{k} \times[0,1] & \stackrel{i}{\rightarrow} & l_{2} \times U \times \Delta^{k} \times[0,1] \\
\hat{p} \mid \downarrow & & \downarrow \text { projection } \\
U \times \Delta^{k} \times[0,1] & = & U \times \Delta^{k} \times[0,1] .
\end{array}
$$

It follows that $l_{2} \times U \times \Delta^{k} \times[0,1] \subset^{i} M^{\prime} \times \Delta^{k} \times[0,1] \stackrel{\tilde{p}}{\rightarrow} U \times \Delta^{k} \times[0,1]$ describes a $(k+1)$-simplex of $M A F(U)$ representing a $(k+1)$-simplex of $M A F(X)$ which is the required lift of $\alpha$.

We now show that $r: \overline{G M A F}(X) \rightarrow \overline{G M A F}(Y)$ is Kan. Let $U$ and $V$ be open as above and assume additionally that $T V \subset T U$. Let $T_{1} U$ be a tangent bundle neighborhood for $U$ such that for some neighborhood $V_{1}$ of $Y$ with $V_{1} \subset U$, we have $T_{1} U \mid V_{1} \subset T V$. 
Suppose we are given a lifting problem. Thus we have a $(k+1)$-simplex of $\overline{G M A F}(Y)$ represented by

$$
l_{2} \times T V_{1} \times \Delta^{k} \times[0,1] \supset N \stackrel{q}{\rightarrow} T V_{1} \times \Delta^{k} \times[0,1]
$$

and a $k$-simplex of $\overline{G M A F}(X)$ represented by

$$
l_{2} \times T U \times \Delta^{k} \supset N \stackrel{p}{\rightarrow} T \underset{\downarrow}{\downarrow} \underset{U \times \Delta^{k}}{\downarrow}
$$

where $p=q$ over $T V_{1} \times \Delta^{k} \times\{0\}$.

Let $E=(M \times\{0\}) \cup N \subset l_{2} \times T U_{1} \times \Delta^{k} \times[0,1]$ and define

$$
f: E \rightarrow\left(T_{1} U \times \Delta^{k} \times\{0\}\right) \cup\left(T_{1} U \mid V_{1} \times \Delta^{k} \times[0,1]\right)
$$

by using $p$ and $q$.

Let $R: U \times \Delta^{k} \times[0,1] \rightarrow\left(U \times \Delta^{k} \times\{0\}\right) \cup\left(\tilde{Y} \times \Delta^{k} \times[0,1]\right)$ be a retraction, fibre preserving over $\Delta^{k}$, where $\tilde{Y}$ is some closed neighborhood of $Y$ contained in $V_{1}$. Let $\tilde{R}: T_{1} U \times \Delta^{k} \times[0,1] \rightarrow\left(T_{1} U \times \Delta^{k} \times\{0\}\right) \cup\left(T_{1} U \mid V_{1} \times \Delta^{k} \times[0,1]\right)$ be a bundle map covering $R$. Form the pull-back

$$
\begin{array}{ccc}
\tilde{E} & \stackrel{\tilde{f}}{\rightarrow} & T_{1} U \times \Delta^{k} \times[0,1] \\
\downarrow & & \\
E & \stackrel{f}{\rightarrow} & \left(T_{1} U \times \Delta^{k} \times\{0\}\right) \cup\left(T_{1} U \mid V_{1} \times \Delta^{k} \times[0,1]\right) .
\end{array}
$$

Then $\tilde{f}: \tilde{E} \rightarrow T_{1} U \times \Delta^{k} \times[0,1]$ represents a $(k+1)$-simplex in $\overline{G M A F}(X)$ which is the appropriate lift.

Proposition 3.5. Let $X \subset B$ be a PL cell and let $x \in X$. Then the restrictions $r: M A F(X) \rightarrow M A F(x)$ and $r: \overline{G M A F}(X) \rightarrow \overline{G M A F}(x)$ are homotopy equivalences.

Proof. We first show that $r: M A F(X) \rightarrow M A F(x)$ induces an isomorphism on homotopy groups. Suppose we have a $k$-simplex $\alpha$ of $M A F(x)$ represented by

$$
l_{2} \times U \times \Delta^{k} \supset M \stackrel{p}{\rightarrow} U \times \Delta^{k}
$$

where $U$ is an open neighborhood of $x$ in $B$. Suppose also that we have a lift of $\partial \alpha$ to $(k+1)(k-1)$-simplices $\beta$ of $M A F(X)$ represented by

$$
l_{2} \times V \times \partial \Delta^{k} \supset N \stackrel{q}{\rightarrow} V \times \partial \Delta^{k}
$$

where $V$ is an open neighborhood of $X$ in $B$ containing $U$ and $p=q$ over $U \times \partial \Delta^{k}$.

Choose an open neighborhood $U_{0}$ of $x$ such that $\operatorname{cl}\left(U_{0}\right) \subset U$. Let $h_{t}: B \rightarrow$ $B, 0 \leq t \leq 1$, be an isotopy such that $h_{0}=\mathrm{id} ; X \subset h_{1}(U) ; h_{t} \mid U_{0}=\mathrm{id}$ and 
$h_{t} \mid B-V=$ id for each $t$. This isotopy defines a homeomorphism $h: B \times$ $[0,1] \rightarrow B \times[0,1]$, and also a self-homeomorphism of $B \times \Delta^{k} \times[0,1]$ which we also denote by $h$.

The diagram

$$
\begin{aligned}
l_{2} \times h\left(U \times \Delta^{k} \times[0,1]\right) \stackrel{\mathrm{id} \times h}{\longleftarrow} l_{2} \times U \times \Delta^{k} \times[0,1] & M \times[0,1] \\
& p \times \text { id } \downarrow \\
& U \times \Delta^{k} \times[0,1] \\
h \downarrow & h\left(U \times \Delta^{k} \times[0,1]\right)
\end{aligned}
$$

represents a $(k+1)$-simplex $\gamma$ of $M A F(x)$. Over $h\left(U \times \Delta^{k} \times\{0\}\right)=U \times \Delta^{k} \times$ $\{0\}$, this diagram represents $\alpha$. Over

$$
\left(h_{1}(U) \times \partial \Delta^{k} \times[0,1]\right) \cup\left(h_{1}(U) \times \Delta^{k} \times\{1\}\right),
$$

this diagram represents a $k$-simplex $\delta$ of $M A F(X)$ such that $\partial \delta=\beta$. Moreover, $\gamma$ shows that $r(\delta) \cong \alpha$ rel $\partial$.

We now show that $r: \overline{G M A F}(X) \rightarrow \overline{G M A F}(x)$ induces an isomorphism on homotopy groups. Let $U$ and $V$ be as above, but assume additionally that $T V \mid U=T U$. Let $h_{t}: B \rightarrow B, 0 \leq t \leq 1$, be the isotopy given above. Lift this isotopy to an isotopy $\tilde{h}_{t}: T V \rightarrow T V, 0 \leq t \leq 1$. Thus $p_{1} \tilde{h}_{t}=h_{t} p_{1}$. Suppose we are given a $k$-simplex $\alpha$ of $\overline{G M A F}(x)$ represented by

$$
l_{2} \times T U \times \Delta^{k} \supset M \stackrel{p}{\rightarrow} T U \times \Delta^{k}
$$

and suppose that we have a lift of $\partial \alpha$ to $\beta$ in $\overline{G M A F}(X)$ represented by

$$
\begin{aligned}
l_{2} \times T V \times \partial \Delta^{k} \supset N \stackrel{q}{\rightarrow} T V & \times \partial \Delta^{k} \\
& \downarrow \\
V & \times \partial \Delta^{k}
\end{aligned}
$$

where $q=p$ over $T U \times \partial \Delta^{k}$. As before we can construct a $(k+1)$-simplex of $\overline{G M A F}(X)$ represented by $M \times[0,1] \stackrel{p \times \text { id }}{\rightarrow} T U \times \Delta^{k} \times[0,1] \stackrel{\tilde{h} \times \text { id }}{\rightarrow} T V \times \Delta^{k} \times[0,1]$ and this $(k+1)$-simplex will show that $r$ induces an isomorphism on homotopy groups.

Proposition 3.6. For each $x \in B, d: M A F(x) \rightarrow \overline{G M A F}(x)$ is a homotopy equivalence.

Proof. We show that $d$ induces an isomorphism on homotopy groups. Let $U$ be an open subset of $B$ containing $x$ such that $U$ is homeomorphic to $\mathbf{R}^{i}$ and $T U=U \times U$. Suppose we are given a $k$-simplex $\alpha$ of $\overline{G M A F}(x)$ represented by the following $k$-simplex of $\overline{G M A F}(U)$ :

$$
l_{2} \times U \times U \times \Delta^{k} \supset M \underbrace{\stackrel{f}{\rightarrow}}_{\rho} \underset{\substack{\downarrow \\ U \times p_{1} \times \mathrm{id} \\ U \times \Delta^{k}}}{U \times \Delta^{k}}
$$


Suppose also that we have a $(k+1)$-simplex $\beta$ of $M A F(x)$ represented by

$$
l_{2} \times U \times \partial \Delta^{k} \supset N \stackrel{p}{\rightarrow} U \times \partial \Delta^{k}
$$

such that $d(\beta)=\partial \alpha$. We can assume that $\rho^{-1}\left(U \times \partial \Delta^{k}\right)=U \times N$ and that $f=\operatorname{id}_{U} \times \rho$ over $U \times U \times \partial \Delta^{k}$.

Let $M_{x}=\rho^{-1}\left(\{x\} \times \Delta^{k}\right)$ and find a homeomorphism $h: U \times M_{x} \rightarrow M$ such that

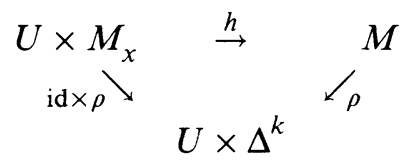

commutes; $h \mid\{x\} \times M_{x}=\mathrm{id}$; and $h \mid U \times \rho^{-1}\left(\{x\} \times \partial \Delta^{k}\right)$ is id: $U \times N \rightarrow U \times N$.

Let $r_{s}: U \times[0,1] \rightarrow U, 0 \leq s \leq 1$, be a strong deformation retraction of $U$ to $x$, and define $f_{s}: U \times M_{x} \rightarrow U \times U \times \Delta^{k}, 0 \leq s \leq 1$, by $f_{s}(y, z)=$ $\left(y, \operatorname{proj}\left(f h\left(r_{s}(y), z\right)\right)\right)$ where proj: $U \times U \times \Delta^{k} \rightarrow U \times \Delta^{k}$ denotes projection onto the last two factors. Use $f_{s}, 0 \leq s \leq 1$, to define $\tilde{f}: U \times M_{x} \times[0,1] \rightarrow$ $U \times U \times \Delta^{k} \times[0,1]$ in the obvious way.

Let $j: U \times M_{x} \times[0,1] \rightarrow l_{2} \times U \times \Delta^{k} \times[0,1]$ be an embedding such that $j\left|U \times M_{x} \times\{0\}=h ; j\right| U \times M_{x} \times\{1\}=$ id ; and $j \mid U \times N \times[0,1]=$ id. Let $\bar{M}$ be the image of $U \times M_{x} \times[0,1]$ in $l_{2} \times U \times \Delta^{k} \times[0,1]$ via $j$. Then we have a $(k+1)$-simplex of $\overline{G M A F}(x)$ represented by

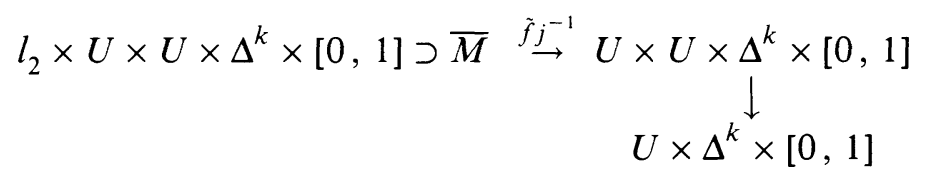

which shows that there is a $k$-simplex $\gamma$ of $M A F(x)$ such that $\partial \gamma=\beta$ and $d(\gamma) \cong \alpha \operatorname{rel} \partial$.

From Gromov's immersion theoretic machine we conclude that $d: M A F(X)$ $\rightarrow \overline{G M A F}(X)$ is a homotopy equivalence for each closed subset of $B$. In particular we have

Theorem 3.7. The differential $d: M A F(B) \rightarrow \overline{G M A F}(B)$ is a homotopy equivalence.

\section{Disassembly}

In this section we give conditions under which a simplicial set is homotopy equivalent to a simplicial set of lifts. The simplicial sets to which this applies are those which are the images of our differentials.

We first recall some standard notation. The $k$-simplex is the span of $\left\{v_{0}, \ldots\right.$, $\left.v_{k}\right\}$ where $v_{0}, \ldots, v_{k}$ are linearly independent points in $l_{2}$ (which we actually fixed in $\S 2$ but the particular choice is irrelevant here). Define boundaries 
and degeneracies as usual as the linear maps $\delta_{i}: \Delta^{k-1} \rightarrow \Delta^{k}$ and $\sigma_{i}: \Delta^{k+1} \rightarrow \Delta^{k}$ defined by setting

$$
\delta_{i}\left(v_{j}\right)=\left\{\begin{array}{ll}
v_{j} & \text { if } j<i, \\
v_{j+1} & \text { if } j \geq i,
\end{array} \text { and } \quad \sigma_{i}\left(v_{j}\right)= \begin{cases}v_{j} & \text { if } j \leq i \\
v_{j-1} & \text { if } j>i\end{cases}\right.
$$

The simplicial set $\Delta[k]$ has $q$-simplices of the form $\left(a_{0}, \ldots, a_{q}\right)$ where $a_{i} \in \mathbb{Z}$ and $0 \leq a_{0} \leq \cdots \leq a_{q} \leq k$ (see [28, p. 14]).

Next we fix some notation which will be used throughout this section. Let $U$ be a space and let $G$ be a simplicial group of homeomorphisms of $U$; i.e., $G$ is the singular complex of a group of homeomorphisms of $U$.

Suppose $K(U)$ is a Kan simplicial set with the following properties:

(i) each $k$-simplex of $K(U)$ is a nonempty subset of $l_{2} \times U \times \Delta^{k}$ (but not all subsets need be simplices)

(ii) if $M \subset l_{2} \times U \times \Delta^{k}$ is a $k$-simplex of $K(U)$ and $0 \leq i \leq k$, then $\partial_{i} M \subset l_{2} \times U \times \Delta^{k-1}$ and $s_{i} M \subset l_{2} \times U \times \Delta^{k+1}$ are defined by $\partial_{i} M=$ $\left\{(x, y, z) \mid\left(x, y, \delta_{i}(z)\right) \in M\right\}$ and $s_{i} M=\left\{(x, y, z) \mid\left(x, y, \sigma_{i}(z)\right) \in\right.$ $M\}$,

(iii) if $h: U \times \Delta^{k} \rightarrow U \times \Delta^{k}$ is a $k$-simplex of $G$ and $M \subset l_{2} \times U \times \Delta^{k}$ is a $k$-simplex of $K(U)$, then $\left(\operatorname{id}_{l_{2}} \times h\right)(M)$ is a $k$-simplex of $K(U)$.

Note that property (iii) describes a simplicial action of $G$ on $K(U)$.

Examples. Let $U=\mathbf{R}^{i}$ and $G=T O P_{i}$ (where $T O P_{i}$ is the simplicial group of homeomorphisms of $\mathbf{R}^{i}$ which preserve the origin). Then $\operatorname{MAF}\left(\mathbf{R}^{i}\right), \operatorname{Bun}\left(\mathbf{R}^{i}\right)$ and $\operatorname{Hur}\left(\mathbf{R}^{i}\right)$ are all examples of simplicial sets satisfying the three conditions above.

Let $B G$ denote the classifying space for $G$ (denoted $\bar{W}(G)$ in [28, p. 87]). Let $T:[B G]_{q} \rightarrow G_{q-1}$ be the twisting function for the universal $G$-bundle $B G \times{ }_{T} G$ over $B G$ (see [28, p. 88], but note that we are reversing the order of base and fibre in the notation for twisted cartesian products). Since $G$ acts on $K(U)$ we can form the associated twisted cartesian product $B G \times{ }_{T} K(U)$ over $B G$.

Suppose that $p: E \rightarrow X$ is a bundle with fibre $U$ and group $G$ (for the examples mentioned above, $X$ will be a neighborhood of the $i$-dimensional manifold $B$ and $p: E \rightarrow X$ will be the tangent bundle of $B$ pulled-back). We assume that $X$ is an ordered simplicial complex and also think of $X$ as a simplicial set with exactly one nondegenerate simplex for each simplex of $X$. That is, we think of $X$ as a subsimplicial set of its singular chain complex $s X$. There is a classifying map $\tilde{p}: X \rightarrow B G$ for $p: E \rightarrow X$, but its definition depends on a choice of atlas for the bundle. We assume that our atlas is regular [28, p. 77], and normalized [28, p. 75], and fix some more notation. For each 
$q$-simplex $\sigma: \Delta^{k} \rightarrow X$, form the pull-back

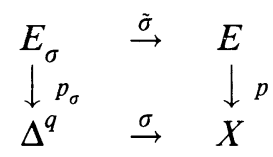

and fix a trivializing homeomorphism $h_{\sigma}: U \times \Delta^{q} \rightarrow E_{\sigma}$.

Normality and regularity tell us how these homeomorphisms behave under boundary and degeneracy operations. To describe this behavior, consider the pull-back diagrams which define $\delta_{i} \sigma$ and $s_{i} \sigma$ :

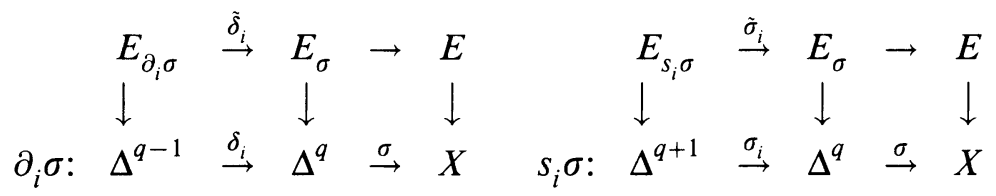

Note that $\tilde{\delta}_{i}: E_{\partial_{i} \sigma} \rightarrow E_{\sigma} \mid \partial_{i} \Delta^{q}=p_{\sigma}^{-1}\left(\partial_{i} \Delta^{q}\right)$ is a homeomorphism. Regularity means that the composition

$$
\begin{aligned}
& U \times \Delta^{q-1} \stackrel{\text { id } \times \delta_{i}}{\rightarrow} U \times \partial_{i} \Delta^{q} \stackrel{h_{\sigma} \mid}{\rightarrow} E_{\sigma} \mid \partial_{i} \Delta^{q} \stackrel{\tilde{\delta}_{i}^{-1}}{\rightarrow} E_{\partial_{i} \sigma} \stackrel{h_{\partial_{i} \sigma}^{-1}}{\rightarrow} U \times \Delta^{q} \\
& \text { is } \begin{cases}\text { id } & \text { if } i>0, \\
T(\tilde{p}(\sigma)) & \text { if } i=0 .\end{cases}
\end{aligned}
$$

Normality means that the following diagram commutes:

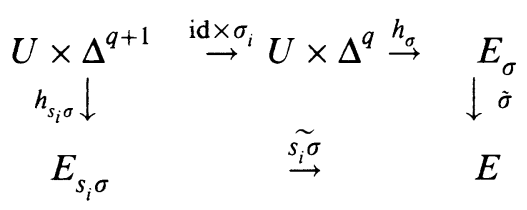

We introduce yet more notation. If $d=(\sigma, \rho)$ is a $q$-simplex of $X \times \Delta[k]$, say $\sigma: \Delta^{q} \rightarrow X$ and $\rho=\left(a_{0}, \ldots, a_{q}\right)$, define the linear map $\hat{\rho}: \Delta^{q} \rightarrow \Delta^{k}$ by setting $\hat{\rho}\left(v_{i}\right)=v_{a_{i}}$. Define $e_{d}: \Delta^{q} \rightarrow X \times \Delta^{k}$ by setting $e_{d}(t)=(\sigma(t), \hat{\rho}(t))$ and define $\tilde{e}_{d}: E_{\sigma} \rightarrow E \times \Delta^{k}$ by $\tilde{e}_{d}(x)=\left(\tilde{\sigma}(x), \hat{\rho}\left(p_{\sigma}(x)\right)\right)$. Thus, the following diagram commutes :

$$
\begin{array}{ccc}
E_{\sigma} & \stackrel{\tilde{e}_{d}}{\rightarrow} & E \times \Delta^{k} \\
p_{\sigma} \downarrow & & \downarrow p \times \mathrm{id} \\
\Delta^{q} & \stackrel{e_{d}}{\rightarrow} & X \times \Delta^{k} .
\end{array}
$$

If $M \subset l_{2} \times U \times \Delta^{q}$, define $M_{d}=\left(\mathrm{id}_{l_{2}} \times \tilde{e}_{d} h_{\sigma}\right)(M) \subset l_{2} \times E \times \Delta^{k}$. If $M C$ $l_{2} \times E \times \Delta^{k}$, define $M^{d}=\left(\operatorname{id}_{l_{2}} \times \tilde{e}_{d} h_{\sigma}\right)^{-1}(M) \subset l_{2} \times U \times \Delta^{q}$.

The goal of this section is to give a workable model for

$$
\operatorname{Lift}\left(\begin{array}{ccc} 
& & B G \times{ }_{T} K(U) \\
& & \downarrow \\
X & \stackrel{\hat{\rho}}{\rightarrow} & B G
\end{array}\right) .
$$


We are now in a position to define a simplicial set $K(p: E \rightarrow X)$ which will turn out to be simplicially isomorphic to the space of lifts, at least for the examples that we have in mind. The $k$-simplices of $K(p: E \rightarrow X)$ are those subsets $M$ of $l_{2} \times E \times \Delta^{k}$ which have the property that $M^{d}$ is a $q$-simplex of $K(U)$ whenever $d$ is a $q$-simplex of $X \times \Delta[k]$. Boundaries and degeneracies are defined as for $K(U)$. Note that $K(p: E \rightarrow X)$ is Kan.

The next step is to define the disassembly map

$$
D: K(p: E \rightarrow X) \longrightarrow \operatorname{Lift}\left(\begin{array}{ccc} 
& B G \times_{T} K(U) \\
& \stackrel{\hat{\rho}}{\rightarrow} & B G
\end{array}\right) .
$$

First note that a $k$-simplex of the lift space is a simplicial map $f: X \times \Delta[k] \rightarrow$ $B G \times{ }_{T} K(U)$ of the form $f=(\hat{\rho}, g)$ where $g: X \times \Delta[k] \rightarrow K(U)$ is a graded map of degree 0 , but not necessarily simplicial. In fact, the following lemma gives necessary and sufficient conditions on $g$ for $(\hat{\rho}, g)$ to be simplicial. The proof is a straightforward chase through the definitions in [28] and is left to the reader.

Lemma 4.1. If $g: X \times \Delta[k] \rightarrow K(U)$ is a degree 0 graded function, then $(\hat{\rho}, g)$ : $X \times \Delta[k] \rightarrow B G \times{ }_{T} K(U)$ is simplicial iff

(i) $g \partial_{i}=\partial_{i} g$ for $i>0$,

(ii) $g \partial_{0}=T(\hat{\rho}(x)) \circ \partial_{0} g(x)$ for $x \in X \times \Delta[k]$, and

(iii) $g s_{i}=s_{i} g$ for $i \geq 0$.

We will call a function $g: X \times \Delta[k] \rightarrow K(U)$ satisfying the conditions of Lemma 4.1. $T$-simplicial and confuse lifts with $T$-simplicial functions.

Now define

$$
D: K(p: E \rightarrow X) \rightarrow \operatorname{Lift}\left(\begin{array}{ccc} 
& B G \times{ }_{T} K(U) \\
& \downarrow \\
X & \stackrel{\hat{\rho}}{\rightarrow} & B G
\end{array}\right)
$$

as follows. If $M \subset l_{2} \times E \times \Delta^{k}$ is a $k$-simplex of $K(p: E \rightarrow X)$, then $D(M): X \times$ $\Delta[k] \rightarrow K(U)$ is the $T$-simplicial function defined by setting $D(M)(d)=M^{d}$ for each $q$-simplex $d$ of $X \times \Delta[k]$. Verification of the following lemma is left to the reader.

Lemma 4.2. $D(M): X \times \Delta[k] \rightarrow K(U)$ is a $T$-simplicial function and

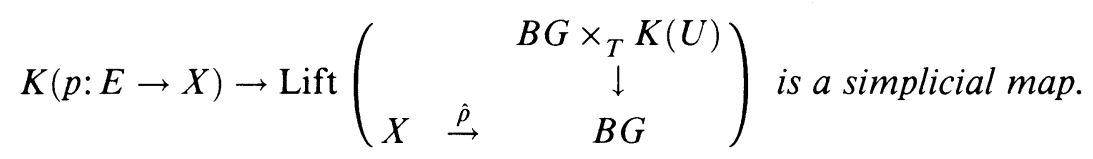

Define the assembly map

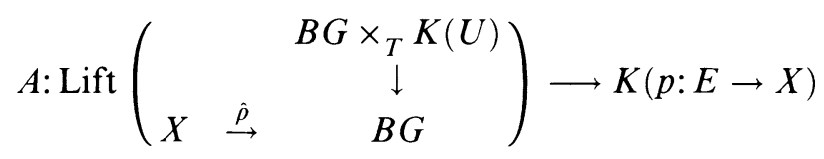


as follows: if $g: X \times \Delta[k] \rightarrow K(U)$ is a $T$-simplicial function, define $A(g)=$ $\bigcup\left\{[g(d)]_{d} \mid d \in X \times \Delta[k]\right\} \subset l_{2} \times E \times \Delta^{k}$. Of course, we need to see that $A(g)$ is a $k$-simplex of $K(p: E \rightarrow X)$. We do this via two lemmas.

Lemma 4.3. If $f$ is an r-simplex of $X \times \Delta[k]$, then $\left([g(f)]_{f}\right)^{f}=g(f)$.

Proof. In general, $g(f) \subset\left([g(f)]_{f}\right)^{f}$ so we only need show that $\left([g(f)]_{f}\right)^{f} \subset$ $g(f)$. This is clear if $f$ is nondegenerate, so assume that $f=s_{i} d$. For $i>0$ we have

$$
\left([g(f)]_{s_{i}} d^{s_{i} d}=\left(\left[\partial_{i} g(f)\right]_{d}\right)^{s_{i} d}=\left(\left[g\left(\partial_{i} f\right)\right]_{d}\right)^{s_{i} d}=s_{i}\left(\left[g\left(\partial_{i} f\right)\right]_{d}\right)^{d}=s_{i}\left([g(d)]_{d}\right)^{d}\right.
$$

which is contained in $s_{i} g(d)=g\left(s_{i} d\right)=g(f)$ by induction. If $i=0$, then the proof above has a twist in it, but nevertheless works.

Lemma 4.4. $A(g)$ is a $k$-simplex of $K(p: E \rightarrow X)$.

Proof. We need to show that $\left(\bigcup_{d} g(d)_{d}\right)^{f}$ is an $r$-simplex of $K(U)$ whenever $f$ is an $r$-simplex of $X \times \Delta[k]$. For this we will show that $\left(\bigcup_{d} g(d)_{d}\right)^{f}=g(f)$. It suffices to show that $\left[g(d)_{d}\right]^{f} \subset g(f)$ for each $q$-simplex of $X \times \Delta[k]$. By Lemma 4.3 we just need to show that $\left[g(d)_{d}\right]^{f} \subset\left[g(f)_{f}\right]^{f}$. This is left to the reader.

We also leave the proof of the following lemma to the reader.

Lemma 4.5. The assembly map $A$ is a simplicial map.

We are now ready for the main result of this section.

Theorem 4.6. The assembly map $A$ is a simplicial isomorphism with inverse the disassembly map $D$.

Proof. To see that $A D(M)=M$ whenever $M \subset l_{2} \times E \times \Delta^{k}$ is a $k$-simplex of $K(p: E \rightarrow X)$, simply note that $M=\bigcup_{d}\left[M^{d}\right]_{d}$.

To see that $D A(g)=g$ whenever $g: X \times \Delta[k] \rightarrow K(U)$ is $T$-simplicial, we have to show that $D A(g)(f)=g(f)$ for each $r$-simplex $f$ of $X \times \Delta[k]$. This amounts to showing that $\left(\bigcup_{d} g(d)_{d}\right)^{f}=g(f)$, which was done in the proof of Lemma 4.4.

Before describing our principal examples, we pause to introduce a construction that we shall need to compare the $M A F$ situation with the Hur situation.

Definition 4.7. Let $p: M \rightarrow B \times X$ be an approximate fibration for which the projection to the $X$ factor is a fibration. Let $\widehat{M}=\left\{(m, \lambda) \in M \times B^{I} \mid p(m)=\right.$ $\left.\left(\lambda(0), p_{X}(m)\right)\right\}$, where $p_{X}$ denotes $p$ followed by the projection to $X$. It follows from Lemma 16.3 that $\hat{p}(m, \lambda)=\left(\lambda(1), p_{X}(m)\right)$ is a Hurewicz fibration. In Definition 9.1, we show how to embed $\widehat{M}$ in $l_{2} \times B \times X$ so that this construction actually induces a simplicial map from $\overline{M A F}(B)$ to $\overline{H u r}(B)$. We refer to this construction as the fibrewise associated Hurewicz fibration. 
Example 4.8. Let $U=\mathbf{R}^{i}, G=T O P_{i}$, and let $K(U)$ be $M A F\left(\mathbf{R}^{i}\right)$. Let $B$ be an $i$ dimensional manifold and let $p: T B \rightarrow B$ be the tangent bundle. Then $K(p: T B \rightarrow B)$ is $\overline{M A F}(B)$. Let $N$ be the total space of a normal disc bundle for $B$. Then $N$ is PL and we have maps $i: B \rightarrow N$ and $r: N \rightarrow B$ with $r \circ i=\mathrm{id}_{B}$ and $i \circ r \cong \mathrm{id}_{N}$. The following composition is a homotopy equivalence:

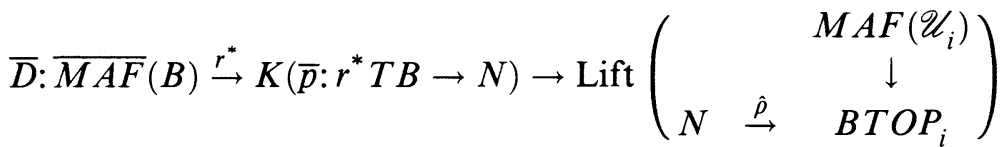

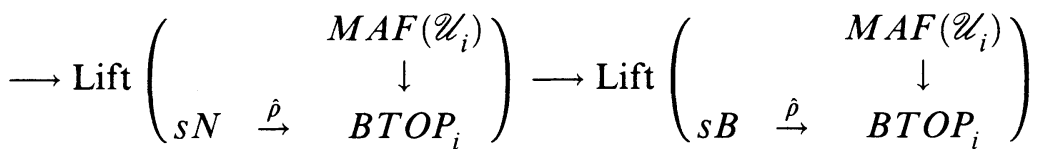

where $s B$ or $s N$ denotes the singular complex of $B$ or $N$ and where $M A F\left(\mathscr{U}_{i}\right)$ denotes the total space of the bundle $B T O P_{i} \times_{T} M A F(U)$.

To see this, recall that

$$
\operatorname{Lift}\left(\begin{array}{ccc} 
& & M A F\left(\mathscr{U}_{i}\right) \\
& \stackrel{\hat{\rho}}{\rightarrow} & B T O P_{i}
\end{array}\right)
$$

is a homotopy functor, so that a simplicial homotopy equivalence induces a homotopy equivalence between the simplicial sets of lifts. Hence all the maps in the above composition after $D$ are equivalences, and $D$ is also an equivalence. Hence we need only show that $r^{*}$ is an equivalence. We show that $i^{*}$ is the homotopy inverse.

Clearly $i^{*} \circ r^{*}$ is the identity on $\overline{M A F}(B)$ so we need only show that $r^{*} \circ i^{*}$ is an equivalence on $K\left(\bar{p}: r^{*} T B \rightarrow N\right)$. It is easy to see that $K(p: E \rightarrow X)$ has maps induced by maps $X \rightarrow Y$, so it is a standard argument that $r^{*} \circ i^{*}$ is an equivalence since it is homotopic to the identity once we show that the two inclusions $N \rightarrow N \times[0,1]$ induce equivalences on $\overline{M A F}$.

To see this, observe that $D$ is natural for simplicial maps and that these inclusions are simplicial. Since the result is clear on the spaces of lifts, we get it for $\overline{M A F}$.

Example 4.9. Let $U=\mathbf{R}^{i}, G=T O P_{i}$, and let $K(U)$ be one of $B u n\left(\mathbf{R}^{i}\right)$ or $\operatorname{Hur}\left(\mathbf{R}^{i}\right)$. Let $B$ be an $i$-dimensional manifold. Let $p: T B \rightarrow B$ be the tangent bundle. Then $K(p: T B \rightarrow B)$ is $\overline{B u n}(B)$ or $\overline{H u r}(B)$, respectively, and as above, we have homotopy equivalences

$$
\begin{aligned}
& \bar{D}: \overline{\operatorname{Bun}}(B) \longrightarrow \operatorname{Lift}\left(\begin{array}{ccc} 
& & B u n\left(\mathscr{U}_{i}\right) \\
& & \downarrow \\
s B & \stackrel{\hat{\rho}}{\rightarrow} & B T O P_{i}
\end{array}\right) \\
& \bar{D}: \overline{\operatorname{Hur}}(B) \longrightarrow \operatorname{Lift}\left(\begin{array}{ccc} 
& & \operatorname{Hur}\left(\mathscr{U}_{i}\right) \\
& & \downarrow \\
s B & \stackrel{\hat{\rho}}{\rightarrow} & B T O P_{i}
\end{array}\right)
\end{aligned}
$$


where $B u n\left(\mathscr{U}_{i}\right)$ denotes the total space of the bundle $B T O P_{i} \times{ }_{T} B u n(U)$, with a similar definition for $\operatorname{Hur}\left(\mathscr{U}_{i}\right)$. We also have a commutative diagram

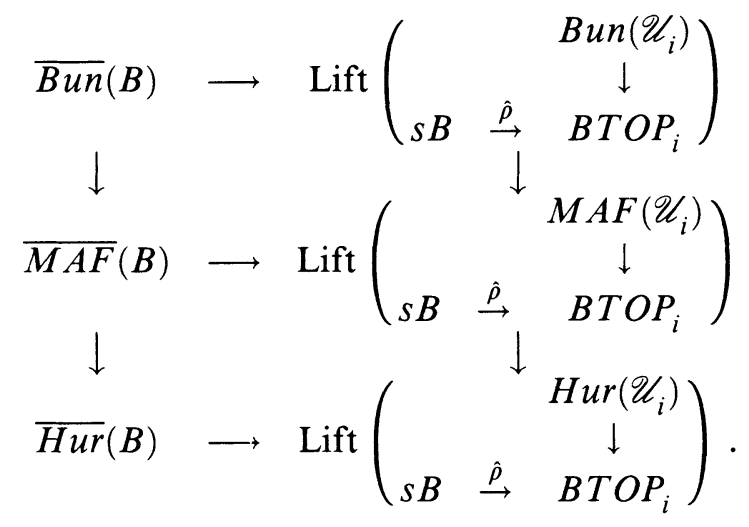

The maps from $\overline{M A F}$ to $\overline{H u r}$ and $M A F\left(\mathscr{U}_{i}\right)$ to $\operatorname{Hur}\left(\mathscr{U}_{i}\right)$ are given by the fibrewise associated Hurewicz fibration after we notice that the fibrewise associated Hurewicz fibration $\operatorname{MAF}\left(\mathbf{R}^{i}\right) \rightarrow \operatorname{Hur}\left(\mathbf{R}^{i}\right)$ is $T O P_{i}$-equivariant.

Finally, Theorem 10.1 shows that $B u n(B)$ and $\operatorname{Hur}(B)$ are homotopy functors. In particular, the restriction maps $B u n\left(\mathbf{R}^{i}\right) \rightarrow B u n(p t)$ and $\operatorname{Hur}\left(\mathbf{R}^{i}\right) \rightarrow$ $H u r(p t)$ are homotopy equivalences which are $T O P_{i}$ equivariant where $T O P_{i}$ acts trivially on $B u n(p t)$ and $\operatorname{Hur}(p t)$. Hence the bundle $B u n\left(\mathscr{U}_{i}\right) \rightarrow B T O P_{i}$ is homotopy equivalent to the trivial bundle with fibre $B u n(p t)$, so the lift simplicial set can be replaced by the space of simplicial maps, $\operatorname{Map}(s B, B u n(p t))$. By Corollary 7.14, the components of $B u n(p t)$ are just $B T O P(N)$ for various manifolds $N$. A similar discussion shows that we may replace the simplicial set of lifts in the Hur case by $M a p(s B, H u r(p t))$, and that the components of $H u r(p t)$ are just $B G(F)$ for the various spaces $F$. These results are wellknown, but we have rederived them to better understand their relation with the $M A F$ case.

\section{Simplicial CATEGORIES AND BI-SIMPLICIAL SETS}

We begin by recalling the definition of a simplicial object. There is a category, $\Delta$, whose objects are $\underline{0}, \ldots, \underline{n}, \ldots$. The morphisms from $\underline{n}$ to $\underline{m}$ consists of the set of all nondecreasing maps from the ordered set $0, \ldots, n$ to the ordered set $0, \ldots, m$. A simplicial object in the category $\mathbf{D}$ is a functoi $\Delta^{\mathrm{op}} \rightarrow \mathbf{D}$. A simplicial category is a functor $\Delta^{\mathrm{op}} \rightarrow$ cat, where cat is the category of small categories. A bisimplicial set can be regarded either as a simplicial object in the category of simplicial sets, or as a functor $\Delta^{\mathrm{op}} \times \Delta^{\mathrm{op}} \rightarrow$ sets, where sets denotes the category of sets.

From a simplicial category, $\mathscr{C}$, we can derive a bisimplicial set $\mathscr{C}_{*, *}$. For each $r$ we have a category, $\mathscr{C}(\underline{r})$. The set $\mathscr{C}_{0, r}$ is the set of objects in $\mathscr{C}(\underline{r})$. If $s \geq 1$, then the set we want for $\mathscr{C}_{s, r}$ is an ordered collection of composible morphisms in $\mathscr{C}(\underline{r})$ with $s$ elements in the collection. 
The horizontal $r$-line, $\mathscr{C}_{*, r}$ is the simplicial set obtained by taking the nerve of the category $\mathscr{C}(\underline{r})$. The vertical 0 -line is the easiest of the vertical lines to describe. It is the simplicial set obtained by just considering the objects in the simplicial category.

There are boundary maps $\partial_{i}^{v}: \mathscr{C}_{r, *} \rightarrow \mathscr{C}_{r-1, *}$ for $0 \leq i \leq r$ with the corresponding degeneracies $s_{i}^{v}$ going back the other way. We call these maps the horizontal boundaries and degeneracies because they connect the vertical lines. There are corresponding maps $\partial_{i}^{h}$ and $s_{i}^{h}$ connecting the horizontal lines, called the vertical boundaries and degeneracies.

We can now describe the sort of result for which we are heading. We consider the simplicial set $\mathscr{C}_{0, *}$, the vertical 0 -line, and fix a vertex, say $\mathbf{v}$. We would like to be able to identify the path component of $\mathscr{C}_{0, *}$ containing $\mathbf{v}$ as follows. Associated to $\mathbf{v}$ there is a simplicial monoid, End $(\mathbf{v})$, defined as follows. For each $k \geq 0$ there is a unique object $\mathbf{v}_{k}$ in $\mathscr{C}_{0, k}$ defined by applying a $k$-fold composition of degeneracies to $\mathbf{v}$. (In a simplicial set the object that one gets is independent of which $k$-fold composite one chooses to apply.) A $k$-simplex in $\operatorname{End}(\mathbf{v})$ is now defined to be a map in $\mathscr{C}_{1, k}$ from $\mathbf{v}_{k}$ to itself.

Recall the construction for $B E n d(\mathbf{v})$ as the diagonal of a bisimplicial set. The $k$-simplices of $E n d(\mathbf{v})$ form a monoid under composition and we can convert $E n d(\mathbf{v})$ into a simplicial category with objects $\mathbf{v}_{k}$ and with the morphisms from $\mathbf{v}_{k}$ to itself being the $k$-simplices in $\operatorname{End}(\mathbf{v})$. Then $B E n d(\mathbf{v})$ is just the diagonal of the bisimplicial set associated to this simplicial category.

Since the simplicial category associated to $\operatorname{End}(\mathbf{v})$ has an obvious inclusion functor to $\mathscr{C}$, there is always a simplicial map

$$
\operatorname{BEnd}(\mathbf{v}) \rightarrow \Delta \mathscr{C}_{*, *}
$$

Under this map, the unique vertex in $B E n d(\mathbf{v})$ is sent to the image of $\mathbf{v}$ in $\Delta \mathscr{C}_{*, *}$.

One goal is to prove that, for the examples which interest us, the map $(*)$ induces a homotopy equivalence between $B E n d(\mathbf{v})$ and the path component of $\Delta \mathscr{C}_{*, *}$ which contains the image of $\mathbf{v}$. The second major goal is to prove that, again for the examples which are of interest to us, the natural map

$$
\mathscr{C}_{0, *} \rightarrow \Delta \mathscr{C}_{*, *}
$$

is a homotopy equivalence. We can then combine these two results to see that the component of $\mathscr{C}_{0, *}$ containing $\mathbf{v}$ is homotopy equivalent to $B E n d(\mathbf{v})$.

We will show that these two results hold provided that our original simplicial category satisfies three additional properties described below. Before taking up the argument, we pause to introduce our examples.

\section{GeOMETRIC EXAMPles of Simplicial CATEgories}

We let $l_{2}$-Top denote the category of subspaces of Hilbert space of small capacity, where the morphisms are just the continuous functions. As before define a countable collection of points, $v_{i}, i=0,1, \ldots$, by $\nu_{i}\left(v_{j}\right)=\delta_{i, j+1}$. There is 
a functor from the category of finite sets and nondecreasing maps, $\Delta$, to $l_{2}-T o p$ given by sending $\{0, \ldots, k\}$ to the convex set spanned by $\left\{v_{0}, \ldots, v_{k}\right\}$ (which of course is a $k$-simplex and will be denoted $\Delta^{k}$ ). We send a nondecreasing map to the corresponding linear map between the two convex sets.

If we begin with a functor $\mathscr{F}: l_{2}-T o p^{\mathrm{op}} \rightarrow$ cat, where cat is the category of small categories, the composite, $\Delta^{\mathrm{op}} \rightarrow l_{2}-T o p^{\mathrm{op}} \rightarrow$ cat , is a simplicial category, $\mathscr{C}(\mathscr{F})$. As we shall see, all the simplicial categories that we will consider in this paper arise from this procedure. It is not true that all such categories satisfy the theorems that we want. To achieve this we will have to demonstrate that our categories satisfy certain additional properties listed below.

We conclude this section with a short guide to applying these results in the cases of interest to us. In all of our categories, the set of objects will be some subset of the following. Fix some space $B$, and consider B-Space: $l_{2}-T o p^{\mathrm{op}} \rightarrow$ cat defined as follows.

An object of B-Space $(X)$ is a subspace $M \subset l_{2} \times B \times X$ of small capacity and a map $p: M \rightarrow B \times X$ such that the following hold.

(i)

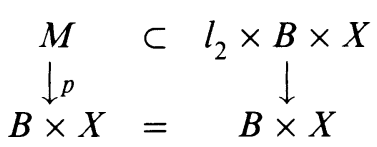

commutes where the right-hand vertical map is just projection on the last two factors.

(ii) Let $p_{X}: M \rightarrow B \times X \rightarrow X$ denote the obvious composite. Then $p_{X}$ is a regular fibration [11]. (Note that every fibration is regular if $B$ is reasonable: e.g. metric.)

The morphisms in B-Space $(X)$ from $\left(M_{1}, p_{1}\right)$ to $\left(M_{2}, p_{2}\right)$ are just the continuous functions $f: M_{1} \rightarrow M_{2}$ which satisfy $\pi_{X} \circ p_{1}=\pi_{X} \circ p_{2} \circ f$, where $\pi_{X}: B \times X \rightarrow X$ is the evident projection.

Given a map $\phi: Y \rightarrow X$, the induced functor $\phi^{*}: \mathbf{B}-\operatorname{Space}(X) \rightarrow \mathbf{B - S p a c e}(Y)$ is defined as follows. We define $\phi^{*}$ first on objects, so let $(M, p)$ be an object as above. Define a subspace of $M \times Y, \bar{M}$, as usual: $(m, y) \in \bar{M}$ iff $p_{X}(m)=$ $\phi(y)$. There is certainly a map $\bar{M} \rightarrow B \times Y$.

There is also a map $M \times Y \rightarrow l_{2} \times B \times Y$ defined as the product of two maps: the first $M \rightarrow l_{2} \times B$ being the inclusion $M \subset l_{2} \times B \times X$ followed by the projection on the first two factors; the second being the projection $M \times Y \rightarrow Y$. Define $\phi^{*}(M)$ to be the subspace which is the image of $\bar{M}$ under this map. The required properties are easily checked.

Given a morphism $f: M_{1} \rightarrow M_{2}$ define $\phi^{*}(f): \phi^{*}\left(M_{1}\right) \rightarrow \phi^{*}\left(M_{2}\right)$ by just restricting the obvious map $M_{1} \times Y \rightarrow M_{2} \times Y$ to the $\phi^{*}\left(M_{i}\right)$.

It is easy to check that these constructions induce a functor from $l_{2}-T o p^{\text {op }}$ to cat. We need only note that $\left(\phi_{1}^{*} \circ \phi_{2}^{*}\right)=\left(\phi_{1} \circ \phi_{2}\right)^{*}$ and that $(\mathrm{id})^{*}=\mathrm{id}$.

We extend the simplicial set $M A F(B)$ to $\mathbf{M A F}(B): l_{2}-$ Top $^{\mathrm{op}} \rightarrow$ cat as follows. An object in $\mathbf{M A F}(B)(X)$ is an object in B-Space $(X)$ for which the 
projection $p_{X}$ is a fibre bundle with compact manifold fibre and for which $p \mid: p^{-1}(B \times x) \rightarrow B \times x$ is an approximate fibration for each $x \in X$. A morphism from $\left(M_{1}, p_{1}\right)$ to $\left(M_{2}, p_{2}\right)$ in $\operatorname{MAF}(B)(X)$ is a controlled homeomorphism $h^{c}: M_{1} \rightarrow M_{2}$ such that each map $h_{t}^{c}$ is a fibre map. Given a map $\phi: Y \rightarrow X$, the functor $\phi^{*}: \operatorname{MAF}(B)(X) \rightarrow \operatorname{MAF}(B)(Y)$ is defined just as above. Again, the needed verifications are straightforward.

We can also extend the simplicial set $\operatorname{Bun}(B)$ to $\operatorname{Bun}(B): l_{2}-T o p^{\text {op }} \rightarrow$ cat . We require that $p$ be a fibre bundle. We require that a morphism be a fibre map which is a homeomorphism.

Similarly, we can extend the simplicial set $\operatorname{Hur}(B)$ to $\operatorname{Hur}(B): l_{2}-T_{o p}{ }^{\text {op }} \rightarrow$ cat. This time we require that $p$ as well as $p_{X}$ be a regular fibration. We require that a morphism be a fibre map for the projections into $B \times X$ (not just for the projections into $X$ ) which is a fibre homotopy equivalence.

Finally, we can define $\mathbf{H A F}(B): l_{2}-T o p^{\text {op }} \rightarrow$ cat . An object in $\operatorname{HAF}(B)(X)$ is an object in B-Space $(X)$ for which $p$ is a fibration, and a morphism is a controlled homotopy equivalence. Of course, we require that each level of the controlled map, $f_{t}^{c}$, be a fibre map with respect to the projections to $X$.

Notice that $\operatorname{Hur}(B)(X)$ is a subcategory of $\operatorname{HAF}(B)(X)$ if we agree to consider a fibre map in Hur as the same thing as a controlled map which is constant at each level. Indeed, these two categories have the same objects.

We have introduced HAF to facilitate the construction of various maps. Finally notice that there is an obvious extension of the simplicial sets $K\left(\tau_{B}: T B \rightarrow B\right)$ to $\overline{\mathbf{M A F}}(B): l_{2}-T o p^{\mathrm{op}} \rightarrow$ cat .

\section{Properties of GeOMETRIC SIMPlicial SETS}

We suppose that we have a functor $\mathscr{F}: l_{2}-T o p^{\text {op }} \rightarrow$ cat. The first property that we wish our functors to satisfy is:

(7.1) Amalgamation Property. We say that $\mathscr{F}$ has the amalgamation property provided the following condition holds for any two subsets $X_{1}$ and $X_{2}$ of $l_{2}$ of small capacity such that the pair $\left(X_{1} \cup X_{2}, X_{1} \cap X_{2}\right)$ is a strong NDR-pair with $X_{1}$ and $X_{2}$ closed in $X_{1} \cup X_{2}$. If the functors in the following square are the ones induced by the inclusions, then the following square commutes and is a pull-back:

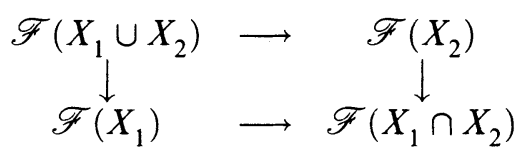

Being a pull-back means that given objects $E_{i}$ in $\mathscr{F}\left(X_{i}\right)$ which are equal when restricted to $\mathscr{F}\left(X_{1} \cap X_{2}\right)$, there is a unique object in $\mathscr{F}\left(X_{1} \cup X_{2}\right)$ which restricts to each $E_{i}$. There is a similar condition on morphisms. In the sequel we will often speak of an object $E$ over $X$ when we mean that $E$ is an object in the category $\mathscr{F}(X)$ : we will also speak of morphisms over $X$. The terminology comes from our bundle/fibration examples where the terminology is standard.

For the notion of an NDR-pair, the reader may consult [42, Chapter 1, §7]. Our first result is the following lemma. 
Lemma 7.2. If $\mathscr{F}$ has the Amalgamation Property each of the vertical simplicial sets $\mathscr{C}(\mathscr{F})_{k, *}$ is a Kan complex.

Proof. Let us begin by showing $\mathscr{C}(\mathscr{F})_{0, *}$ is Kan. The Kan condition requires that we can do the following. We begin with $k$ objects, say $E_{0}, \ldots, E_{i-1}, E_{i+1}$, $\ldots, E_{k}$ over the $(k-1)$-simplex which satisfy the compatibility condition $\partial_{n} E_{j}=\partial_{j-1} E_{n}, n<j, n \neq i, j \neq i$. We must construct an object over the $k$-simplex, say $E$, such that $\partial_{n} E=E_{n}$ for $n \neq i$ [28, Definition 1.3, p. 2].

Let $w_{i} \Delta^{k}$ denote the subcomplex of $\Delta^{k}$ consisting of all faces that do not contain the $i$ th vertex. Then the compatibility condition on the $E_{i}$ and the Amalgamation Property guarantee that there is an object $\bar{E}$ over $w_{i} \Delta^{k}$ so that the restriction to the face $\partial_{n} \Delta^{k}$ of $\Delta^{k}$ is just $E_{n}$.

There is a map $r: \Delta^{k} \rightarrow w_{i} \Delta^{k}$ which is a retract for the natural inclusion. If we let $E$ denote the object induced over $\Delta^{k}$ from $\bar{E}$ using $r, E$ clearly satisfies the required properties.

To show that $\mathscr{C}(\mathscr{F})_{k, *}, k \geq 1$, is Kan is a similar argument complicated only by the notation since now we must amalgamate and induce maps and their ranges and domains.

The next result that we want is that one of the $\partial_{i}^{v}$ is a Kan fibration. This needs some additional properties of $\mathscr{C}(\mathscr{F})$. We say that objects in $\mathscr{F}$ can be straightened provided the following property holds.

(7.3) Straightening Property. Let $E$ be an object in $\mathscr{F}\left(\Delta^{k} \times[0,1]\right)$. We can restrict $E$ to $\Delta^{k} \times 0$, getting say $E_{0}$. Then we can apply the projection $\Delta^{k} \times$ $[0,1] \rightarrow \Delta^{k} \times 0$ to $E_{0}$ to get another object over $\Delta^{k} \times[0,1]$, say $E_{0} \times[0,1]$. We require that there exist maps $F: E \rightarrow E_{0} \times[0,1]$ and $G: E_{0} \times[0,1] \rightarrow E$ such that $F$ and $G$ restricted to $\Delta^{k} \times 0$ are both the identity map. We say that $\mathscr{F}$ has the Strong Straightening Property if, whenever $E \mid\left(\partial \Delta^{k} \times[0,1]\right)$ is equal to $\left(E_{0} \mid \partial \Delta^{k}\right) \times[0,1]$, we can chose the $F$ and $G$ above so as to be the identity when restricted over $\partial \Delta^{k} \times[0,1]$.

Remark. The reason for the terminology can be found by considering an example. If we consider the category of bundles over $B$, where objects over $X \subset l_{2}$ are just bundles over $B \times X$, we have a functor $\mathscr{F}: l_{2}-T o p^{\text {op }} \rightarrow$ cat. In this case, the Straightening Property just says that any bundle over $B \times X \times[0,1]$ is bundle isomorphic to the bundle restricted to $B \times X \times 0$ crossed with $[0,1]$.

The next result gives the needed Kan fibrations.

Lemma 7.4. Let $\mathscr{F}: l_{2}-$ Top ${ }^{\text {op }} \rightarrow$ cat have the Amalgamation and Straightening Properties. Then the map $\partial_{0}: \mathscr{C}(\mathscr{F})_{1, *} \rightarrow \mathscr{C}(\mathscr{F})_{0, *}$ is a Kan fibration.

Proof. Since we have the Amalgamation Property we can replace the usual statement of the Kan condition by starting with two objects over $w_{i} \Delta^{k}$, say $E_{1}$ and $E_{2}$, together with a map $f: E_{1} \rightarrow E_{2}$. Moreover, the object $E_{1}$ has an 
extension to an object $E$ over all of $\Delta^{k}$. We must find an extension of $E_{2}$ to all of $\Delta^{k}$, say $\bar{E}$, and a map $F: E \rightarrow \bar{E}$ which when restricted to $w_{i} \Delta^{k}$ is just $f$. This is not the usual way to state the Kan fibration condition [28, Definition 7.1, p. 25], but it is equivalent to it in the presence of the Amalgamation Property by an argument similar to that used to prove Lemma 7.2.

There is a homeomorphism $h: \Delta^{k} \rightarrow w_{i} \Delta^{k} \times[0,1]$ which takes $w_{i} \Delta^{k}$ to $w_{i} \Delta^{k} \times 0$ by the identity. By the functorial nature of $\mathscr{F}$ there is an object $\widehat{E}$ over $w_{i} \Delta^{k} \times[0,1]$ and an isomorphism from $E$ to $\widehat{E}$ which is the identity when restricted to $w_{i} \Delta^{k} \times 0$. Hence it suffices to produce the extension over $w_{i} \Delta^{k} \times[0,1]$ and then use $h$ to induce the result back over $\Delta^{k}$.

For $\bar{E}$ we can take $E_{2}$, now over $w_{i} \Delta^{k} \times 0$ and induce it up to $w_{i} \Delta^{k} \times[0,1]$ using the projection. If we let $\bar{E}_{1}$ denote the result of performing the same construction on $E_{1}$, now over $w_{i} \Delta^{k} \times 0$, then the map $f$ extends to a map $\bar{f}$ which restricts to $f$ over $w_{i} \Delta^{k} \times 0$. By the Straightening Property we can find a map $F: \widehat{E} \rightarrow \bar{E}_{1}$ which is the identity when restricted to $w_{i} \Delta^{k} \times 0$. Clearly the composite $\bar{f} \circ F$ is the required map.

We need a further property of the map $\partial_{0}: \mathscr{C}(\mathscr{F})_{1, *} \rightarrow \mathscr{C}(\mathscr{F})_{0, *}$. Given a vertex $\mathbf{v}$ in $\mathscr{C}(\mathscr{F})_{0,0}$ we define a simplicial set $\partial_{0}^{-1}(\mathbf{v})$ as follows. A $k$-simplex in $\partial_{0}^{-1}(\mathbf{v})$ consists of a $k$-simplex $E$ in $\mathscr{C}(\mathscr{F})_{0, *}$ and a map $f: E \rightarrow \mathbf{v}_{k}$ where $\mathbf{v}_{k}$ denotes the $k$-fold degeneracy applied to $\mathbf{v}$. Our next goal is to prove that $\partial_{0}^{-1}(\mathbf{v})$ is contractible. To do this we need to assume that $\mathscr{F}$ has another property.

(7.5) Fill-in Property. Suppose given three objects $E_{0}, E_{1}, E_{2}$ over $\Delta^{k}$, and two maps $f_{i}: E_{i} \rightarrow E_{2}, i=0,1$. Let $E_{2} \times[0,1]$ denote the result of pulling $E_{2}$ back to $\Delta^{k} \times[0,1]$ via the projection to $\Delta^{k} \times 0$. We say that $\mathscr{F}$ has the Fill-in Property provided we can find an object $\bar{E}_{1}$ over $\Delta^{k} \times[0,1]$ such that $\bar{E}_{1}$ restricted over 0 is $E_{0}$ and $\bar{E}_{1}$ restricted over 1 is $E_{1}$. Moreover, we require a map $F: \bar{E}_{1} \rightarrow E_{2} \times[0,1]$ such that $F$ restricted over 0 is $f_{0}$ and $F$ restricted over 1 is $f_{1}$. Finally, the process needs to be mildly functorial. Specifically, suppose we are given two sets of initial data, say the data above and another set of initial data, three objects $\widehat{E}_{0}, \widehat{E}_{1}, E_{2}$ over $\Delta^{k}$, and two maps $\hat{f}_{i}: \widehat{E}_{i} \rightarrow E_{2}, i=0,1$. Suppose that $\widehat{E}_{i}$ and $E_{i}$ are equal when restricted to a collection of faces of $\Delta^{k}$. Moreover, suppose that $\hat{f}_{i}$ and $f_{i}$ agree when restricted to this collection. Then the two extensions and the two extended maps agree when restricted to this collection $\times[0,1]$. Notice that we are given both sets of data before we have to produce either extension and the range object for both sets of maps is the same.

One useful result is

Lemma 7.6. Let $\mathscr{F}: l_{2}-$ Top $^{\mathrm{op}} \rightarrow$ cat have the Amalgamation Property. Let $E_{0}$ and $E_{1}$ be two $k$-simplices such that $E_{i} \mid \partial \Delta^{k}$ is "trivial": i.e. there exists an ob- 
ject over a point which, when pulled-back to $\partial \Delta^{k}$, is equal to $E_{i} \mid \partial \Delta^{k}$. By Lemma 7.4, $\mathscr{C}(\mathscr{F})_{0, *}$ is Kan, so such simplices represent elements in $\pi_{k}\left(\mathscr{C}(\mathscr{F})_{0, *}\right)$.

(a) If $\mathscr{F}$ satisfies the Strong Straightening Property and $\left[E_{0}\right]=\left[E_{1}\right]$ in $\pi_{k}$, then there exist maps $f: E_{0} \rightarrow E_{1}$ and $g: E_{1} \rightarrow E_{0}$ such that $f$ and $g$ are the identity when restricted over $\partial \Delta^{k}$.

(b) If $\mathscr{F}$ has the Fill-in Property and if there exists a third object $E_{2}$ over $\Delta^{k}$ (with no conditions on the $\partial \Delta^{k}$ ) and maps $f_{i}: E_{i} \rightarrow E_{2}, i=0,1$, such that $f_{0}\left|\partial \Delta^{k}=f_{1}\right| \partial \Delta^{k}$ then $\left[E_{0}\right]=\left[E_{1}\right]$ in $\pi_{k}$.

Proof. For part (a), the fact that $\mathscr{C}(\mathscr{F}))_{0, *}$ is Kan implies that we can find an object over $\Delta^{k+1}$ which is $E_{0}$ when restricted to one face, $E_{1}$ when restricted to another, and "trivial" when restricted to the remaining faces. There is a map $\Delta^{k} \times[0,1] \rightarrow \Delta^{k+1}$ which takes $\Delta^{k} \times\{0\}$ to the face under $E_{0}$ and $\Delta^{k} \times\{1\}$ to the face under $E_{1}$. Consider the induced object over $\Delta^{k} \times[0,1]$. Clearly it is $E_{i}$ over $\Delta^{k} \times\{i\} \quad i=0,1$ and "trivial" over $\partial \Delta^{k} \times[0,1]$. The Strong Straightening Property supplies the maps.

For part (b), use the Fill-in Property to construct objects over $\Delta^{k} \times[0,1]$. There is a map $\Delta^{k+1} \rightarrow \Delta^{k} \times[0,1]$ which takes one face of $\Delta^{k+1}$ to $\Delta^{k} \times\{0\}$ homeomorphically; which sends another face of $\Delta^{k+1}$ to $\Delta^{k} \times\{1\} \cup \partial \Delta^{k} \times[0,1]$ homeomorphically, and which collapses the other faces onto lower dimensional simplices in $\partial \Delta^{k}$. If we pull-back the fill-in for $f_{i}$ along this map, we see a homotopy from $\left[E_{i}\right]$ to $E_{2} \cup$ (the fill-in construction for $f_{i}$ restricted to $\left.\partial \Delta^{k} \times[0,1]\right)$. By the uniqueness property for fill-ins, we can arrange for both these constructions to be equal.

The key result that we need is

Lemma 7.7. Let $\mathscr{F}: l_{2}-T_{o p}{ }^{\mathrm{op}} \rightarrow$ cat have the Amalgamation and Fill-in Properties. Then the simplicial set $\partial_{0}^{-1}(\mathbf{v})$ is a contractible Kan complex.

Proof. Extend $\partial_{0}^{-1}(\mathbf{v})$ to $l_{2}-T o p^{\text {op }} \rightarrow$ cat by requiring that an object over $X$ be an object in $\mathscr{F}$ over $X$, say $E$, together with a map $f: E \rightarrow \mathbf{v}_{X}$, where $\mathbf{v}_{X}$ denotes our object over a point induced back to $X$. A map between $\left(E_{0}, f_{0}\right)$ and $\left(E_{1}, f_{1}\right)$ is a map $g: E_{0} \rightarrow E_{1}$ in $\mathscr{F}$ such that $f_{1} \circ g=f_{0}$. It is not hard to see that this extended functor satisfies the Amalgamation and Fill-in Properties.

By Lemma 7.2, $\partial_{0}^{-1}(\mathbf{v})$ is Kan. To show that it is contractible, we show that the homotopy groups are 0 . Let $f: E \rightarrow \mathbf{v}_{k}$ be a $k$-simplex which is "trivial" along the boundary. Notice that $i d: \mathbf{v}_{k} \rightarrow \mathbf{v}_{k}$ is another object in $\partial_{0}^{-1}(\mathbf{v})$, and $f$ extends to a map in $\partial_{0}^{-1}(\mathbf{v})$ between these two objects. The identity extends to a map from id: $\mathbf{v}_{k} \rightarrow \mathbf{v}_{k}$ to itself and hence Lemma 7.6(b) shows that our original element represents the same element in homotopy as id: $\mathbf{v}_{k} \rightarrow \mathbf{v}_{k}$, which is the trivial element.

We can now prove one of our two main results. 
Proposition 7.8. The natural map $\mathscr{C}(\mathscr{F})_{0, *} \rightarrow \Delta \mathscr{C}(\mathscr{F})_{*, *}$ is a homotopy equivalence if $\mathscr{F}$ has the Amalgamation, Straightening and Fill-in Properties.

Proof. This result follows from standard nonsense [39] once we prove that the $k$-fold composite of degeneracies, $\mathscr{C}_{0, *} \rightarrow \mathscr{C}_{1, *} \rightarrow \mathscr{C}_{2, *} \rightarrow \cdots \rightarrow \mathscr{C}_{k, *}$, is a homotopy equivalence. To do this, consider the following square

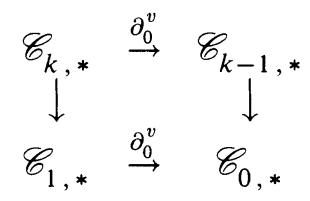

This square is a pull-back : i.e. a $k$-fold collection of maps is equivalent to the first map together with the last $k-1$. By Lemma 7.4, the bottom $\partial_{0}^{v}$ is a Kan fibration, so in the pull-back, the top $\partial_{0}^{v}$ is a homotopy equivalence iff the bottom $\partial_{0}^{v}$ is. But the bottom $\partial_{0}^{v}$ is a homotopy equivalence because the homotopy fibre over a vertex $\mathbf{v}$ is $\partial_{0}^{-1}(\mathbf{v})$, which is contractible by Lemma 7.7.

Since $\partial_{0}^{v}$ is a homotopy equivalence, $s_{0}^{v}$ is the homotopy inverse because $s_{0}^{v} \circ \partial_{0}^{v}$ is the identity. Therefore, any $k$-fold iterate of degeneracies from $\mathscr{C}_{0, *}$ to $\mathscr{C}_{k, *}$ is also a homotopy equivalence.

Remark. Once we know that these iterated degeneracies are homotopy equivalences, we can easily show that any $s_{i}^{v}$, and hence any $\partial_{i}^{v}$ is also a homotopy equivalence.

Lemma 7.9. Let $\mathscr{F}: l_{2}-T o p^{\mathrm{op}} \rightarrow$ cat have the Amalgamation, Straightening, and Fill-in Properties. Then the map $\partial_{1}: \partial_{0}^{-1}(\mathbf{v}) \rightarrow \mathscr{C}(\mathscr{F})_{0, *}$ is a Kan fibration. The fibre $\partial_{1}^{-1}(\mathbf{v})$ is the simplicial monoid $\operatorname{End}(\mathbf{v})$, and $\operatorname{End}(\mathbf{v})$ acts on $\partial_{0}^{-1}(\mathbf{v})$ by composition.

Proof. All of these statements are obvious except the statement that $\partial_{1}$ is Kan. The proof of this is rather similar to the proof of Lemma 7.4. After the same reductions as we did there, we are left with showing the following. We have an object over $\Delta^{k}$, say $E_{1} \rightarrow \mathbf{v} \times \Delta^{k}$ (where $\mathbf{v} \times \Delta^{k}$ represents our vertex $\mathbf{v}$ induced up by the only map from $\Delta^{k}$ to a point). We also have an extension of $E_{1}$ to $\Delta^{k} \times[0,1]$, say $\bar{E}_{1}$. We must construct a map of $\bar{E}_{1}$ to $\mathbf{v} \times \Delta^{k} \times[0,1]$ which extends our map over $\Delta^{k} \times 0$.

To begin, use the Fill-in Property to get an object over $\Delta^{k} \times[0,1]$, say $E_{2}$, which extends $\mathbf{v} \times \Delta^{k}$ and a map of $\bar{E}_{1}$ to it which is our map at the 0 -end. Then use the Straightening Property to get a map of $E_{2}$ to $\mathbf{v} \times \Delta^{k} \times[0,1]$ which is the identity over the 0 -end. The composite of these two gives the desired map.

Next we want to describe the individual components of $\mathscr{C}(\mathscr{F})_{0, *}$ as discussed at the beginning of this section. We begin with a technical lemma which will enable us to use the conclusions of Lemma 7.9. The goal of this lemma is to 
show that, under certain hypotheses, a map $p: E \rightarrow B$ is homotopy equivalent to the standard loop-path fibration $\Omega B \rightarrow P B \rightarrow B$. Our standard is the Moore model: i.e. fix a point $b \in B ; P B=\{\lambda:[0, r] \rightarrow B \mid r \geq 0, \lambda(0)=b\}$ with $\Omega B=\{\lambda \in P B \mid \lambda(r)=b\}$.

Lemma 7.10. Let $p: E \rightarrow B$ be a simplicial map. Suppose that there exists a simplicial monoid $M$ and an action $\mu: E \times M \rightarrow E$ which commutes with the map $p$. In particular, the action map plus a choice of point in $E$ gives a map of $M$ into $E$ and hence of $M$ into the homotopy fibre of $p$. Finally, suppose that $E$ is contractible and that the map of $M$ to the homotopy fibre of $p$ is a homotopy equivalence. Then there is a monoid $M_{1}$ and monoid maps $M_{1} \rightarrow M$, $M_{1} \rightarrow \Omega B$ which are homotopy equivalences. Moreover there is a contractible space $E_{1}$ with a monoid action of $M_{1}$ on it and equivariant maps $E_{1} \rightarrow E$ and $E_{1} \rightarrow P B$. The two composites $E_{1} \rightarrow B$ are equal and $M_{1} \rightarrow E_{1} \rightarrow B$ is a homotopy fibration.

Proof. We begin by proving that our monoid $M$ is group-like : i.e. that $\pi_{0}(M)$ is a group. This is equivalent to showing that right translation by $m \in M$ as a map from $M$ to $M$ is a homotopy equivalence. But this is an easy 5lemma argument since there is a right translation by $m$ on the whole homotopy fibration $M \rightarrow E \rightarrow B$.

For the purposes of this proof, a pseudo-principal fibration is a homotopy fibration with fibre a monoid, an action of this monoid on the total space commuting with the projection, and a contractible total space. All three homotopy fibrations in the conclusion of the lemma are pseudo-principal. A pseudoprinciple map is a map between two pseudo-principal fibrations for which the map on the total spaces covers the map on the base; the induced map of fibres is a monoid map; and the map on the total spaces is equivariant. In the conclusion of the lemma we are also promised two pseudo-principal maps which are homotopy equivalences.

We will leave it to the reader to check that we can achieve the conclusion of the lemma by merely exhibiting a sequence of pseudo-principal fibrations beginning with $M \rightarrow E \rightarrow B$ and ending with $\Omega B \rightarrow P B \rightarrow B$ together with pseudo-principal maps between successive pseudo-principal fibrations in the sequence (not all the maps will go the same way or will be over the same base space). When there is a pseudo-principal map from one pseudo-principal fibration to another which induces homotopy equivalences on the bases, total spaces and fibres, we say that we may replace one pseudo-principal fibration by the other. The usual 5-lemma argument shows that if two of the three maps are equivalences, then so is the third.

Our first goal is to replace $M \rightarrow E \rightarrow B$ by a more tractable object. Given a monoid $M$ and a space $E$ on which $M$ acts, there are two simplicial constructions we may perform. The first, denoted $\bar{W}(E, M)$, is the simplicial set $E \leftleftarrows E \times M \leftleftarrows E \times M \times M \cdots$; the second, denoted $W(E, M)$, is the simplicial set $E \times M \leftleftarrows E \times M \times M \nsubseteq E \times M \times M \times M$ (see 
[28, Chapter IV]). There is an obvious action of $M$ on $W(E, M)$ and a map $W(E, M) \rightarrow \bar{W}(E, M)$. Moreover $W(E, M)$ is contractible and there are maps $W(E, M) \rightarrow E$ and $\bar{W}(E, M) \rightarrow B$. The map $W(E, M) \rightarrow E$ is $M$-equivariant and induces the identity map on a fibre.

There is an equivariant map $E \rightarrow *$ where $*$ denotes a point. Hence we can replace $M \rightarrow W(E, M) \rightarrow \bar{W}(E, M)$ by $M \rightarrow W(*, M) \rightarrow \bar{W}(*, M)$. If $M$ is group-like, $M \rightarrow W(*, M) \rightarrow \bar{W}(*, M)$ is a homotopy fibration. This is the "group completion theorem" of Quillen [29]. Hence, $M \rightarrow W(E, M) \rightarrow$ $\bar{W}(E, M)$ is also a homotopy fibration and an easy 5-lemma argument shows that the map $\bar{W}(E, M) \rightarrow B$ is a homotopy equivalence.

Given any monoid $M$, there is a free monoid, $F$, and a monoid map $F \rightarrow M$ which is a homotopy equivalence (see lemma below). Hence we may replace $M \rightarrow W(*, M) \rightarrow \bar{W}(*, M)$ by $F \rightarrow W(*, F) \rightarrow \bar{W}(*, F)$.

Given any free monoid $F$ there is a monoid map to the group $G=G \bar{W}(*, F)$ obtained by adding inverses. If $M$, and hence $F$, is group-like, the map $F \rightarrow G$ is a homotopy equivalence [28, Remarks 27.5], or [25]. Hence we may replace $F \rightarrow W(*, F) \rightarrow \bar{W}(*, F)$ by $G \rightarrow W(*, G) \rightarrow \bar{W}(*, G)$, where this last sequence of simplicial sets is actually a principal $G$-bundle.

There are many different models for the path space fibration over $B$ and we may start with any of them in which $\Omega B$ is a monoid and acts on $P B$, preserving the projection. If we apply the above construction in this case we will eventually arrive at another principal bundle, say $G_{1} \rightarrow W\left(*, G_{1}\right) \rightarrow \bar{W}\left(*, G_{1}\right)$. Both $\bar{W}\left(*, G_{1}\right)$ and $\bar{W}(*, G)$ are homotopy equivalent to $B$, so we can induce both these bundles back over $B$, where each will be a principal bundle with contractible total space.

There is a model for the path fibration over $B$ in which the fibre is Kan's simplicial group on $B, G(B)$. Furthermore, there is a group map $G(B) \rightarrow G$ and an equivariant map of total spaces [28, Corollary 27.3]. Hence we may replace each of our principal bundles by this model for the path fibration.

Because we were unable to find a reference in the literature we include a proof of the following result. The proof follows Quillen [30, Proposition 3, p. 4.5], rather closely.

Lemma. Let $M$ be a simplicial monoid. Then there exists a free simplicial monoid $F$ and a simplicial monoid homomorphism, $p: F \rightarrow M$, such that the underlying map of simplicial sets is a Kan fibration with contractible fibres.

Proof. Before beginning the proof proper, we recall two useful results. The first is that any map of simplicial sets $f: X \rightarrow Y$ is a Kan fibration with contractible fibres iff given any commutative square

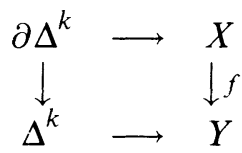

there exists a map $\Delta^{k} \rightarrow X$ making the obvious two triangles commute. 
The second result we need is that $\partial \Delta^{k}$ is "compact" in the sense that, for any filtered direct system of simplicial sets, the natural map

$$
\operatorname{hom}\left(\partial \Delta^{k}, \underset{i}{\lim } X_{i}\right) \longrightarrow \underset{i}{\lim } \operatorname{hom}\left(\partial \Delta^{k}, X_{i}\right)
$$

is an isomorphism of simplicial sets.

To begin the construction of $F$, let $F_{0}$ be the free monoid $\{e\}$, and let $p_{0}: F_{0} \rightarrow M$ be the map which sends $e$ to the identity element of $M$. Given $p_{i}: F_{i} \rightarrow M$, define $p_{i+1}: F_{i+1} \rightarrow M$ as follows. Let $S$ denote the set of all commutative diagrams

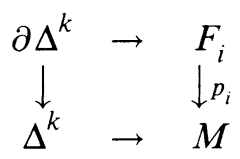

and form the pushout

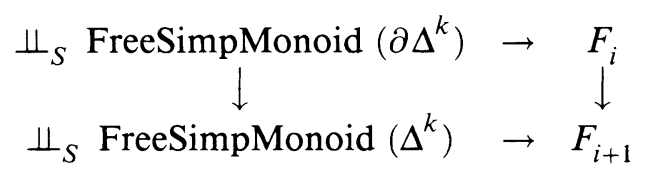

where FreeSimpMonoid $(X)$ denotes the free monoid on the simplicial set $X$, and the top horizontal map is the map induced by the simplicial set map $\partial \Delta^{k} \rightarrow$ $F_{i}$, which is part of the structure of an element of $S$. There is a monoid homomorphism $p_{i+1}: F_{i+1} \rightarrow M$ given by using the rest of the structure of each element of $S$, and one can verify easily that $F_{i+1}$ is a free simplicial monoid.

Let $F=\lim _{i} F_{i}$. There is a map $p: F \rightarrow M$ which we show satisfies our criterion for a map to be a Kan fibration with contractible fibres. Given

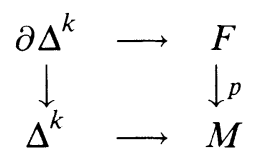

there exists an $i$ such that our square comes from

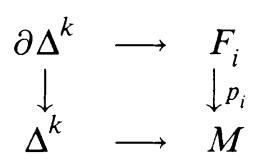

by our second remark above. But our construction guarantees that the required map $\Delta^{k} \rightarrow F$ actually exists already at the $(i+1)$ st level.

We can now prove one of the main results of this section.

Theorem 7.11. Let $\mathscr{F}: l_{2}-T o p^{\mathrm{op}} \rightarrow$ cat have the Amalgamation, Straightening, and Fill-in Properties. The Kan fibration $\partial_{1}: \partial_{0}^{-1}(\mathbf{v}) \rightarrow \mathscr{C}(\mathscr{F})_{0, *}$ has contractible total space and is acted on by End $(\mathbf{v})$. Hence the component of $\mathscr{C}(\mathscr{F})_{0, *}$ containing $\mathbf{v}$ is homotopy equivalent to $B E n d(\mathbf{v})$. Indeed, the natural maps 
$\mathscr{C}(\mathscr{F})_{0, *} \rightarrow \Delta \mathscr{C}(\mathscr{F})_{*, *}$ and $\Perp B E n d(\mathbf{v}) \rightarrow \Delta \mathscr{C}(\mathscr{F})_{*, *}$ are homotopy equivalences.

Proof. Lemmas 7.7 and 7.9 show that $\partial_{1}: \partial_{0}^{-1}(\mathbf{v}) \rightarrow \mathscr{C}(\mathscr{F})_{0, *}$ is a Kan fibration with contractible total space acted on by $\operatorname{End}(\mathbf{v})$, which is the fibre of $\partial_{1}$. Hence, by Lemma 7.10, the component of $\mathscr{C}(\mathscr{F})_{0, *}$ containing $\mathbf{v}$ is homotopy equivalent to $B E n d(\mathbf{v})$.

As we saw in the proof of Lemma 7.7, we can extend the simplicial set, $\partial_{0}^{-1}(\mathbf{v})$, to an $l_{2}-T o p^{\text {op }} \rightarrow$ cat functor. An object in $\partial_{0}^{-1}(\mathbf{v})(X)$ is an object over $X$, say $E$, and a map $E \rightarrow \mathbf{v}_{X}$ where $\mathbf{v}_{X}$ represents our vertex induced up by the unique map from $X$ to a point. A morphism in $\partial_{0}^{-1}(\mathbf{v})(X)$ between $f_{1}: E_{1} \rightarrow \mathbf{v}_{X}$ and $f_{2}: E_{2} \rightarrow \mathbf{v}_{X}$ is a map $g: E_{1} \rightarrow E_{2}$ such that $f_{2} \circ g=f_{1}$. Notice that $\partial_{0}^{-1}(\mathbf{v})(X)$ has a terminal object so the nerve of this category is contractible. This means that $\Delta \partial_{0}^{-1}(\mathbf{v})_{*, *}$ is contractible. Notice that the monoid $\operatorname{End}(\mathbf{v})$ continues to act on $\Delta \partial_{0}^{-1}(\mathbf{v})_{*, *}$ and the map from $\partial_{0}^{-1}(\mathbf{v})_{0, *}$ to $\Delta \partial_{0}^{-1}(\mathbf{v})_{*, *}$ is equivariant. Another application of Lemma 7.10 shows that $\Perp B E n d(\mathbf{v}) \rightarrow$ $\Delta \mathscr{C}(\mathscr{F})_{*, *}$ is a homotopy equivalence.

It follows from Proposition 7.8 that the map $\mathscr{C}(\mathscr{F})_{0, *} \rightarrow \Delta \mathscr{C}(\mathscr{F})_{*, *}$ is a homotopy equivalence.

We wish to show that our categories satisfy our three properties.

Amalgamation. Hur satisfies the Amalgamation Property by Lemma 16.2. The fibration condition is also the key point to check for HAF .

In the bundle category Bun, the Amalgamation Property holds since we can reduce to the trivial bundle case and then patch the trivializations together. This bundle condition is also the only condition that needs to be checked in MAF. The simplicial functor $\overline{\mathbf{M A F}}$ also has the Amalgamation Property since the result can be easily reduced to the Bun case.

Straightening. The Strong Straightening Property for Bun is classical if the bundle is numerable. This is always the case if $B \subset l_{2}$. For MAF it is the Approximation Theorem of [20, p. 168], after we apply Lemma 12.11 to show that the $\varepsilon-\delta$ condition there is equivalent to our definition here (see $\S 14)$. Notice that the dimension of the total space manifold must be at least 5. For Hur and for HAF, the Strong Straightening Property is a straightforward consequence of the homotopy lifting property.

Fill-in. In Bun, fill-ins can be constructed using mapping cylinders. For Hur, the Fill-in Property is checked by Lemma 17.3. For MAF, it is checked by Lemma 17.4. For HAF it is checked by Lemma 17.5. These results require that $B$ be reasonable: $B \subset l_{2}$ will certainly do.

We apply the previous material to our examples. The proofs are the same. Theorem 7.11 identifies the path components and Lemma 7.6 gives the equivalences. There is even a version of the equivalence statements that is true over $\Delta^{k}$. 
Corollary 7.12. Let $p: M \rightarrow B$ be an approximate fibration with $M$ and $B$ manifolds, with the dimension of $M$ at least 5. Then the component of $M A F(B)$ containing $p$ is homotopy equivalent to $B T o p^{c}(p: M \rightarrow B)$ via the maps discussed above, where $T o p^{c}$ denotes the simplicial group of controlled homeomorphisms from $p: M \rightarrow B$ to itself. Given two manifold approximate fibrations $p_{0}: M_{0} \rightarrow B$ and $p_{1}: M_{1} \rightarrow B$, they are in the same path component of $M A F(B)$ iff they are controlled homeomorphic.

Corollary 7.13. If $p: E \rightarrow B$ is a fibration with $B$ a manifold, then the component of $H A F(B)$ containing $p$ is homotopy equivalent to $B G^{c}(p: E \rightarrow B)$ via the maps discussed above, where $G^{c}$ denotes the simplicial group of controlled homotopy equivalences of $p: E \rightarrow B$ to itself. Given two fibrations $p_{0}: E_{0} \rightarrow B$ and $p_{1}: E_{1} \rightarrow B$, they are in the same path component of $H A F(B)$ iff they are controlled homotopy equivalent.

Corollary 7.14. If $p: E \rightarrow B$ is a fibre bundle with $B$ a manifold, then the component of Bun $(B)$ containing $p$ is homotopy equivalent to $B T o p^{f}(p: E \rightarrow B)$ via the maps discussed above, where Top ${ }^{f}$ denotes the simplicial group of fibre homeomorphisms of $p: E \rightarrow B$ to itself. Given two fibre bundles $p_{0}: E_{0} \rightarrow B$ and $p_{1}: E_{1} \rightarrow B$, they are in the same path component of $B$ un $(B)$ iff they are fibrewise homeomorphic.

Corollary 7.15. If $p: E \rightarrow B$ is a fibration with $B$ a manifold, then the component of $\operatorname{Hur}(B)$ containing $p$ is homotopy equivalent to $B G^{f}(p: E \rightarrow B)$ via the maps discussed above, where $G^{f}$ denotes the simplicial group of fibre homotopy equivalences of $p: E \rightarrow B$ to itself. Given two fibrations $p_{0}: E_{0} \rightarrow B$ and $p_{1}: E_{1} \rightarrow B$, they are in the same path component of $H u r(B)$ iff they are fibre homotopy equivalent.

\section{FUNCTORS BETWEEN GEOMETRIC SIMPLICIAL CATEGORIES}

The main result in this section is to give a description of the homotopy fibre of a simplicial map $\zeta: \mathscr{C}\left(\mathscr{F}_{1}\right)_{0, *} \rightarrow \mathscr{C}\left(\mathscr{F}_{2}\right)_{0, *}$ for two functors $\mathscr{F}_{i}: l_{2}-T$ op op $\rightarrow$ cat , $i=1,2$. We begin by fixing a vertex $\mathbf{v}$ in $\mathscr{C}\left(\mathscr{F}_{2}\right)_{0, *}$ and we wish to describe the homotopy fibre of $\zeta$ over $\mathbf{v}$, assuming that it is nonempty. Let $\mathbf{v}_{k}$ denote the result of inducing $\mathbf{v}$ up to $\Delta^{k}$ via the iterated degeneracy.

(8.1) The Homotopy Fibre. Define a simplicial set $\mathscr{S}(\zeta, \mathbf{v})$ as follows. A $k$ simplex in $\mathscr{S}(\zeta, \mathbf{v})$ consists of a $k$-simplex, $E$, in $\mathscr{C}\left(\mathscr{F}_{1}\right)_{0, k}$ and a map $f: \zeta(E) \rightarrow \mathbf{v}_{k}$ in $\mathscr{C}\left(\mathscr{F}_{2}\right)_{1, k}$.

Since $\zeta$ commutes with boundaries and degeneracies, it is not hard to check that $\mathscr{S}(\zeta, \mathbf{v})$ inherits boundaries and degeneracies from $\mathscr{C}\left(\mathscr{F}_{1}\right)_{0, *}$ and $\mathscr{C}\left(\mathscr{F}_{2}\right)_{1, *}$ and so it is a simplicial set. There is a natural simplicial map $\mathscr{S}(\zeta, \mathbf{v}) \rightarrow \mathscr{C}\left(\mathscr{F}_{1}\right)_{0, *}$ defined by sending the simplex $(E, f)$ to $E$. 
Proposition 8.2. Let $\zeta$ be a simplicial map $\zeta: \mathscr{C}\left(\mathscr{F}_{1}\right)_{0, *} \rightarrow \mathscr{C}\left(\mathscr{F}_{2}\right)_{0, *}$ where $\mathscr{F}_{i}: l_{2}-$ Top $^{\mathrm{op}} \rightarrow$ cat $, i=1,2$, is a functor. Suppose that $\mathscr{F}_{2}$ has the Amalgamation, Straightening and Fill-in Properties. Then the sequence of simplicial maps

$$
\mathscr{S}(\zeta, \mathbf{v}) \rightarrow \mathscr{C}\left(\mathscr{F}_{1}\right)_{0, *} \rightarrow \mathscr{C}\left(\mathscr{F}_{2}\right)_{0, *}
$$

is a homotopy fibration and $\mathscr{S}(\zeta, \mathbf{v})$ is Kan. The monoid End(v) acts on $\mathscr{S}(\zeta, \mathbf{v})$ and this monoid action is equivalent to the usual homotopy action of $\Omega \mathscr{C}\left(\mathscr{F}_{2}\right)_{0, *}$ on $\mathscr{S}(\zeta, \mathbf{v})$.

Proof. Consider the following diagram

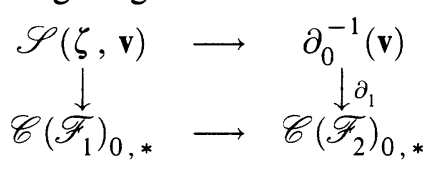

This diagram clearly commutes and is a pull-back of simplicial sets. By Theorem $7.11, \partial_{1}$ is $\mathrm{Kan} ; \partial_{0}^{-1}(\mathbf{v})$ is contractible; and $\operatorname{End}(\mathbf{v})$ acts.

Hence the map $\mathscr{S}(\zeta, \mathbf{v}) \rightarrow \mathscr{C}(\mathscr{F})_{0, *}$ is Kan and it is easy to see that $\mathscr{S}(\zeta, \mathbf{v})$ $\rightarrow \mathscr{C}\left(\mathscr{F}_{1}\right)_{0, *} \rightarrow \mathscr{C}\left(\mathscr{F}_{2}\right)_{0, *}$ is a homotopy fibration. The composition action of $\operatorname{End}(\mathbf{v})$ on $\mathscr{S}(\zeta, \mathbf{v})$ is identical to the action of $E n d(\mathbf{v})$ on the pull-back, so $\Omega \mathscr{C}\left(\mathscr{F}_{2}\right)_{0, *}$ acts on $\mathscr{S}(\zeta, \mathbf{v})$ via the $E n d(\mathbf{v})$ action. By [28, Proposition 7.5(ii), p. 26], $\mathscr{S}(\zeta, \mathrm{v})$ is Kan.

\section{The equivalence of HAF AND Hur}

We begin this section with a definition of a useful variant of the usual Hurewicz fibration.

Definition 9.1. There is a simplicial map from $\mathscr{C}(\mathbf{M A F}(B))_{0, *}$ to $\mathscr{C}(\mathbf{H u r}(B))_{0, *}$ defined as the restriction of a transformation, $T$, from the objects in $\operatorname{MAF}(B)(X)$ to the objects in $\operatorname{Hur}(B)(X)$, which is natural for maps $\phi: X \rightarrow Y$. Given $(M, p)$ an object in B-Space $(B \times X)$, form $\widehat{M} \subset M \times B^{I}$ where $(m, \lambda) \in \widehat{M}$ iff $p(m)=\left(\lambda(0), p_{X}(m)\right)$. Define a map $\hat{p}: \widehat{M} \rightarrow B \times X$ by $\hat{p}(m, \lambda)=\left(\lambda(1), p_{X}(m)\right)$. There exists an embedding of $l_{2}^{I}$ into $l_{2}$ which we fix once and for all. This induces an embedding of $B^{I}$ into $l_{2}$ since $B$ is a manifold and hence has an embedding into $l_{2}$ that we also fix. There is then an obvious embedding of $\widehat{M}$ in $l_{2} \times B \times X$. Let $E_{X}(M)$ denote the image of $\widehat{M}$ : let $E_{X}(p)$ denote the composite of the homeomorphism $E_{X}(M) \rightarrow \widehat{M}$ followed by $\hat{p}$. Then Lemma 16.3 says that $\hat{p}$, and hence $E_{X}(p)$, is a fibration and this is the only part of checking that $\left(E_{X}(M), E_{X}(p)\right)$ is an object in $\operatorname{Hur}(B)(X)$ that is not straightforward. It is also not hard to check that $E_{X} \circ \phi$ equals $\phi \circ E_{X}$, so $E_{X}$ does induce a simplicial map $\mathscr{C}(\mathbf{M A F}(B))_{0, *} \rightarrow \mathscr{C}(\operatorname{Hur}(B))_{0, *}$. On the vertex level, these two simplicial maps just take an approximate fibration to its associated Hurewicz fibration, but over a $k$-simplex with $k>0$ we get a variant of the standard construction. We will refer to this variant as the fibrewise-associated Hurewicz fibration. 
Remark. In fact, as long as $B$ embeds in $l_{2}$, the above discussion defines a simplicial map from

$$
\mathscr{C}(\mathbf{B}-\operatorname{Space}(B))_{0, *} \rightarrow \mathscr{C}(\mathbf{H u r}(B))_{0, *} .
$$

Next we prove that controlled homotopy equivalences are equivalent to fibre homotopy equivalences for a fibration. To describe one direction of the equivalence, we introduce the following construction. Let $p: E \rightarrow B$ be a fibration in $\operatorname{Hur}(B)\left(\mathbf{v}_{0}\right)$. In particular, $p$ is regular, so let us fix a regular solution to the universal lifting problem, say $F: E(p) \times[0,1] \rightarrow E$. Any controlled map $f: E \times[0,1) \rightarrow E$, gives rise to a map $\hat{f}: E \rightarrow E(p)$ since $f$ completes to a map $E \times[0,1] \rightarrow B$. A quick check shows that the composite $F(\hat{f}(), 1): E \rightarrow E$ is a fibre map, hence a fibre homotopy equivalence by Dold's theorem. Another check shows that, since $F$ is a regular solution, if $f$ is a constant family of fibre maps, then we obtain the same fibre map back from our construction.

Now consider the fibration $p \times$ id: $E \times \Delta^{k} \rightarrow B \times \Delta^{k}$. A controlled map over $\Delta^{k}$ is already fibre-preserving over $\Delta^{k}$ and so gives a map $E \times \Delta^{k} \rightarrow E(p) \times \Delta^{k}$. We can use $F \times$ id as above to recover a fibre map from $E \times \Delta^{k}$ to itself. It has the property that if the original controlled map was a constant family of fibre maps then we recover this map. Furthermore, this construction yields a simplicial map from the monoid of controlled self-equivalences of $p$ to the monoid of fibre homotopy equivalences of $p$.

Warning. We do not claim that this map is a monoid map.

Proposition 9.2. For a fixed fibration $p: E \rightarrow B$ in $\operatorname{Hur}(B)\left(\mathbf{v}_{0}\right)$, the inclusion $G^{f}(p) \subset G^{c}(p)$ is a monoid map and a homotopy equivalence $\left(G^{f}(p)\right.$ denotes the fibre-homotopy equivalences of $p$ and $G^{c}(p)$ denotes the controlled homotopy equivalences of $p)$. The simplicial map $G^{c}(p) \rightarrow G^{f}(p)$ constructed above is a retract for the inclusion and hence a homotopy inverse for it.

Proof. By Corollary $7.15, B G^{f}(p)$ is homotopy equivalent to the path component of $\mathscr{C}(\operatorname{Hur}(B))_{0, *}$ containing $p$. Corollary 7.13 describes $B G^{\mathcal{C}}(p)$ as the path component of $\mathscr{C}(\mathbf{H A F}(B))_{0, *}$ containing $p$. But these two simplicial sets are identical. It is a chase through our maps to see that the equivalence given by these remarks is also given by applying $B$ to the monoid map $G^{f}(p) \subset G^{c}(p)$. The rest of the proposition is clear.

\section{BUn AND HUr ARE HOMOTOPY FUNCTORS}

Let $f: B_{1} \rightarrow B_{2}$ be a map. We want to define a simplicial map $f^{*}: B u n\left(B_{2}\right) \rightarrow$ $\operatorname{Bun}\left(B_{1}\right)$. Given a $k$-simplex, $E_{2}$, in $\operatorname{Bun}\left(B_{2}\right)$ we can form the pull-back to get a space $E_{1}$ and a map to $B_{1} \times \Delta^{k}$ which is a fibre bundle. To get a $k$ simplex in $B u n\left(B_{1}\right)$ or $\operatorname{Hur}\left(B_{1}\right)$ we require a subset of $l_{2} \times B_{1} \times \Delta^{k}$. There is a map of $E_{1}$ into $l_{2} \times B_{1} \times \Delta^{k}$ which is just the product of the projection to 
$B_{1} \times \Delta^{k}$ and the composition $E_{1} \rightarrow E_{2} \rightarrow l_{2}$. It is easily checked that this map embeds $E_{1}$ in $l_{2} \times B_{1} \times \Delta^{k}$, and we define $f^{*}\left(E_{2}\right)$ to be this image. It is easy to check that this map is simplicial.

A similar construction works for $\mathrm{Hur}$. We have

Theorem 10.1 (The Bun Hur Theorem). If $B \subset l_{2}$, both Bun and Hur are homotopy functors: i.e. the induced map $f^{*}$ defined above depends up to homotopy only on the homotopy class of $f$. In particular, a homotopy equivalence $f$ induces a homotopy equivalence $f^{*}$.

Proof. The conclusion for $\mathrm{Hur}$ follows from formal manipulations if we can establish that $p: B \times[0,1] \rightarrow B$ induces a homotopy equivalence $p^{*}: \operatorname{Hur}(B) \rightarrow$ $\operatorname{Hur}(B \times[0,1])$. Any inclusion $i: B \rightarrow B \times[0,1]$ is obviously a retraction, so it suffices to prove that $p^{*} \circ i^{*}$ induces the identity on homotopy groups for a fixed $i$. We choose $i$ so that $i(b)=b \times\{0\}$.

All our simplicial sets are Kan, Lemma 7.2, so we can choose a typical homotopy element to be $E \rightarrow B \times[0,1] \times \Delta^{k}$ (with embedding data suppressed) which is equal to some $E_{2} \times \partial \Delta^{k} \rightarrow B \times[0,1] \times \partial \Delta^{k}$ when restricted to $B \times[0,1] \times \partial \Delta^{k}$. Since $H u r$ satisfies the Strong Straightening Property there is a fibration $E_{0} \rightarrow B \times \Delta^{k}$ which is trivial along $B \times \partial \Delta^{k}$ and a map $f: E \rightarrow E_{0} \times[0,1]$, which is the identity along $B \times \partial \Delta^{k} \times[0,1]$. Notice that $p^{*} \circ i^{*}(E)=E_{0} \times[0,1]$. By Lemma 7.6(b), $E$ and $E_{0} \times[0,1]$ represent the same element in $\pi_{k}(\operatorname{Hur}(B \times[0,1]))$, so $p^{*} \circ i^{*}$ induces the identity on homotopy.

A similar argument works for Bun .

We conclude this section with a discussion of the relationship between the usual classification theorem for Bun and $H u r$ and our result, Example 4.9. Both classifications assert that, say bundles with base $B$ and fibre $F$, are given by maps of $B$ into $B T O P(F)$. We would like to claim that these two ways of getting such a map give the same map, at least up to homotopy. The extent to which we realize this desire is given in Theorem 10.5 below.

We begin with a lemma which yields a more direct description of our map $\operatorname{Bun}(B) \rightarrow \Perp \operatorname{Map}(B, B T O P(F))$. Recall the notation from $\S 4$, where we introduced a simplicial set $K(U)$, a simplicial group $G$ which acts on $U$, and a bundle $B G \times{ }_{T} K(U)$. We also introduced another simplicial set $K(p: E \rightarrow X)$, where $p$ is a $G$-bundle over $X$ and defined a disassembly map

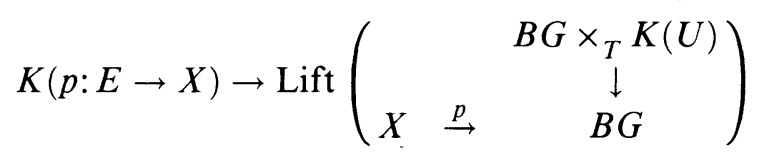

whenever $X$ is an ordered simplicial complex. The proof of the following lemma is a tedious chase through the definitions and is omitted.

Lemma 10.2. Suppose $V \subset U$ is a G-invariant subset. Define $K(V)$ to be the simplicial set whose $k$-simplices are of the form a $k$-simplex in $K(U)$, which 
is a subset of $l_{2} \times U \times \Delta^{k}$, intersected with the subset $l_{2} \times V \times \Delta^{k}$. Associated to the bundle $p$, there is a subbundle $E_{0} \subset E$ with projection denoted $p_{0}$ and fibre $V$. From the definition of $K(p: E \rightarrow X)$ there is defined a restriction map $K(p: E \rightarrow X) \rightarrow K\left(p_{0}: E_{0} \rightarrow X\right)$. There is a G-invariant restriction map $K(U) \rightarrow K(V)$, and hence a map of bundles and hence of spaces of lifts. The following diagram commutes if $X$ is an ordered simplicial complex:

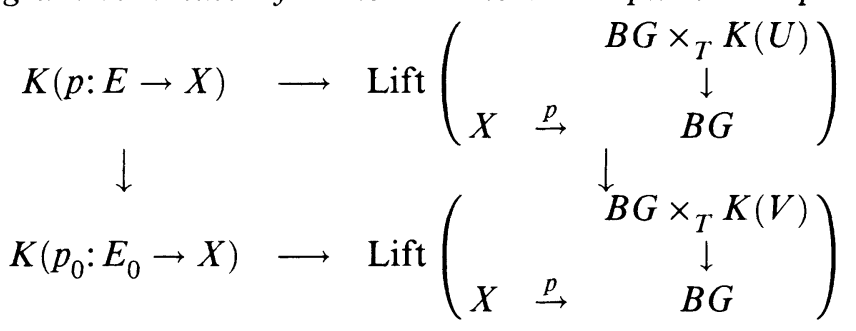

Suppose that $N \subset G$ is a normal simplicial subgroup which acts as the identity on $V$. Then $p_{0}: E_{0} \rightarrow X$ is also a $G / N$ bundle; $G / N$ acts on $K(V)$, and the following diagram commutes:

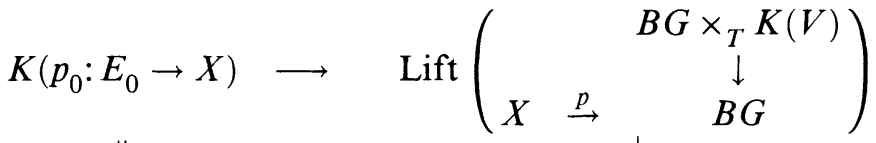

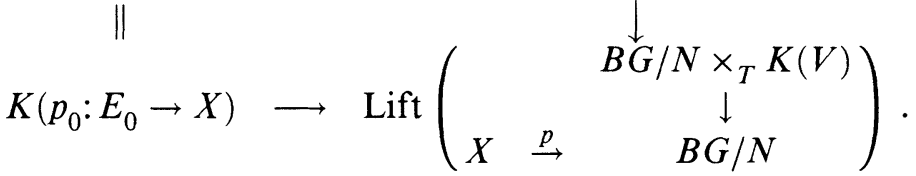

As an application of this lemma, let $K$ be the $K$ associated with either the bundle or fibration case. As in Example 4.9, we take $G=T O P_{i}$ and $U=\mathbf{R}^{i}$ : For $V$ we take the origin, and for $N$ we take $G$ itself. The last space of lifts in Lemma 10.2 just becomes

$$
\operatorname{Lift}\left(\begin{array}{ccc} 
& & * \times{ }_{T} K(V) \\
& & \downarrow \\
X & \stackrel{p}{\rightarrow} & *
\end{array}\right),
$$

which is clearly the same as $\operatorname{Maps}(X, K(V))$. Moreover $p_{0}: E_{0} \rightarrow X$ is just id: $X \rightarrow X$, and $K(\mathrm{id}: X \rightarrow X)$ is equal to $\operatorname{Bun}(X)$ or $\operatorname{Hur}(X)$. (It is this last statement that is false for $M A F$.) Hence, for an ordered simplicial complex, $X$, we have a direct disassembly which does not go through the tangent bundle, but if $X$ is a finite dimensional manifold then our new map is homotopic to the one we discussed in Example 4.9. Moreover, the material in $\S 4$ proves

Lemma 10.3. The disassemblies

$$
\operatorname{Bun}(X) \rightarrow \Perp \operatorname{Maps}(X, B T O P(F))
$$

and

$$
\operatorname{Hur}(X) \rightarrow \Perp \operatorname{Maps}(X, B G(F))
$$

are homotopy equivalences for an ordered simplicial complex $X$. 
Next, suppose that $X_{0} \subset X$ is a subcomplex which is ordered from the ordering on $X$. We have restrictions $\operatorname{Bun}(X) \rightarrow \operatorname{Bun}\left(X_{0}\right)$ and $\operatorname{Hur}(X) \rightarrow$ $\operatorname{Hur}\left(X_{0}\right)$. A chase through the definitions in $\S 4$ shows that

Lemma 10.4. The following diagram commutes:

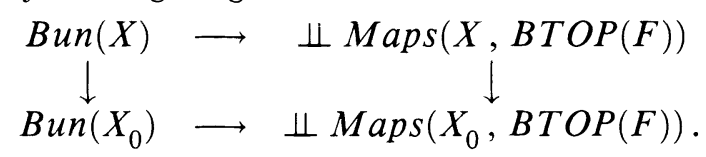

There is a similar diagram for $\mathrm{Hur}$ which also commutes.

Using the homotopy invariance of Bun and Hur together with the above lemmas, it is a standard exercise to extend the definition of the disassembly to any space $X$ which is homotopy equivalent to an ordered simpicial complex so that it remains a homotopy equivalence. Moreover, given any map between such spaces, the diagram analogous to the one in Lemma 10.4 homotopy commutes. We record this result in

Theorem 10.5. Let $X_{0}$ and $X_{1}$ be subspaces of $l_{2}$ which are homotopy equivalent to $C W$ complexes, and let $f: X_{0} \rightarrow X_{1}$ be a map. The diagram below homotopy commutes and the horizontal maps are homotopy equivalences:

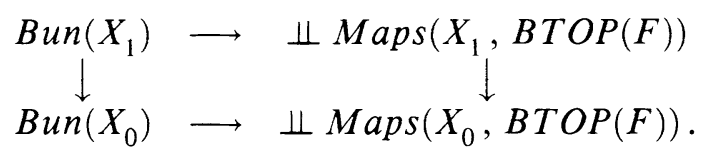

There is a similar result for $\mathrm{Hur}$.

Remark. If either $B T O P(F)$ or $B G(F)$ is homotopy equivalent to a countable $\mathrm{CW}$ complex we may take a model for it which is a subspace of $l_{2}$. Theorem 10.5 shows that the usual classification theorem is equivalent to ours. The identity map $B T O P(F) \rightarrow B T O P(F)$ corresponds to a bundle over $B T O P(F)$, say $\mu$, and any bundle $p: E \rightarrow B$ with fibre $F$ is equivalent to a map $\hat{p}: B \rightarrow$ $B T O P(F)$ given by our procedure. Theorem 10.5 says that $p$ is also given by pulling the bundle $\mu$ back along the map $\hat{p}$, which is the usual description of the classification procedure.

\section{EQUiVALENCES OF SIMPLICIAL SETS OF STRUCTURES}

Recall the definition of the structure set. Fix a fibration $p: E \rightarrow B$. A $k$-simplex of the structure set consists of a $k$-simplex in $M A F(B)$, say $M \rightarrow$ $B \times \Delta^{k}$ (with the subset data suppressed as usual) together with a fibre map which is a controlled homotopy equivalence between $M$ and $E \times \Delta^{k} \stackrel{p \times \text { id }}{\rightarrow} B \times \Delta^{k}$. Let us denote this structure set by $\mathscr{S}(p: E \rightarrow B)$. We have

Theorem 11.1. Let $p: E \rightarrow B$ be a vertex in $H u r(B)$. Then, the homotopy fibre of the map

$$
M A F(B) \rightarrow \operatorname{Hur}(B)
$$

is homotopy equivalent to the simplicial set of structures on $p$. 
Proof. It follows from Proposition 8.2 that the homotopy fibre of this map can be identified with the following simplicial set. A $k$-simplex consists of a $k$-simplex in $M A F(B)$, say $M \rightarrow B \times \Delta^{k}$ (with the subset data suppressed as usual) together with a fibre homotopy equivalence between the fibrewiseassociated Hurewicz fibration for $M$, and $E \times \Delta^{k} \rightarrow B \times \Delta^{k}$. Denote this structure set by $\mathscr{S}_{\text {Hur }}(p: E \rightarrow B)$.

Fix a fibre homotopy equivalence from the associated Hurewicz fibration to $E$, say $\widehat{E}$, to $E$. It is easy to construct a simplicial inclusion $\mathscr{S}(p: E \rightarrow B) \rightarrow$ $\mathscr{S}_{H u r}(p: E \rightarrow B)$ and a simplicial retract $r: \mathscr{S}_{H u r}(p: E \rightarrow B) \rightarrow \mathscr{S}(p: E \rightarrow B)$. We know that $\mathscr{S}_{\text {Hur }}(p: E \rightarrow B)$ is Kan by Proposition 8.2 and $S(p: E \rightarrow B)$ is Kan since it is a retract of a Kan complex.

Next consider the composition

$$
\mathscr{S}(p: E \rightarrow B) \rightarrow \mathscr{S}_{\text {Hur }}(p: E \rightarrow B) \rightarrow M A F(B) .
$$

The image of $\pi_{k}(\mathscr{S}(p: E \rightarrow B))$ in $\pi_{k}(M A F(B))$ is easily seen to be equal to the image of $\left.\pi_{k} \mathscr{S}_{H u r}(p: E \rightarrow B)\right)$ in $\pi_{k}(M A F(B))$. Furthermore the simplicial monoid of automorphisms of $p, E n d(p)$, acts on $\mathscr{S}(p: E \rightarrow B)$ and the simplicial monoid of automorphisms of the associated Hurewicz to $p, E n d(E(p))$, acts on $\mathscr{S}_{H u r}(p: E \rightarrow B)$. Since $p$ and its associated Hurewicz are fibre homotopy equivalent, these two monoids are homotopy equivalent.

Moreover, two elements in $\pi_{k}\left(\mathscr{S}_{H u r}(p: E \rightarrow B)\right)$ which go to the same element in $\pi_{k}(M A F(B))$ differ by the action of some element from $\pi_{k}(E n d(E(p)))$. It is now easy to see that the map $\mathscr{S}(p: E \rightarrow B) \rightarrow \mathscr{S}_{H u r}(p: E \rightarrow B)$ induces an epimorphism on homotopy groups. The retraction of $\mathscr{S}_{H u r}(p: E \rightarrow B)$ onto $S(p: E \rightarrow B)$ shows that both the inclusion and the retraction are actually homotopy equivalences.

\section{APPENDICES ON FibRATIONS, APPROXimAte FIBRATIONS, AND MAF's}

We collect here the necessary prerequisites concerning approximate fibrations.

\section{Definitions AND RESUltS ON APPROXIMATE FIBRATIONS}

Definition 12.1. Let $p_{i}: E_{i} \rightarrow B, i=0,1$, be two spaces over $B$. A controlled map from $p_{0}$ to $p_{1}$ is a map $g: E_{0} \times[0,1) \rightarrow E_{1} \times[0,1)$ which is fibrepreserving over $[0,1)$ and such that the map $\bar{g}: E_{0} \times[0,1] \rightarrow B$, defined by $\bar{g} \mid E_{0} \times[0,1)$ is $p_{1} \circ$ projection $\circ g$ and $\bar{g} \mid E_{0} \times 1$ is $p_{0}$, is continuous. We also denote this by saying that $g^{c}: E_{0} \rightarrow E_{1}$ is a controlled map.

We first show that a controlled map is what Quinn calls an approximate fibre $\operatorname{map}[34,2.7]$.

Lemma 12.2. Let $p_{i}: E_{i} \rightarrow B, i=0,1$, be two spaces over $B$. Assume that $p_{1} \times$ $\mathrm{id}_{[0,1]}$ is closed. Then any controlled map induces a map of mapping cylinders $M\left(p_{0}\right) \rightarrow M\left(p_{1}\right)$ which is level-preserving and which is the identity on $B$. 
Remarks. The converse is obvious with no conditions on $p_{1}$. If $p_{1}$ is proper and $B$ is compactly-generated (e.g. locally-compact) then $p_{1} \times \mathrm{id}_{[0,1]}$ is closed. Some hypotheses are necessary for the conclusion. If we let $B$ be a point, $E_{0}=[0,1]$ and $E_{1}=(0,1)$, then there is a controlled map $f: E_{0} \times[0,1) \rightarrow E_{1}$ defined by $f(s, t)=(s+1-t) /(4-3 t)$. It is not hard to check that this does not extend to a level-preserving, continuous function of the mapping cylinders.

Proof. To fix notation, let $\pi: E_{1} \times[0,1] \rightarrow M\left(p_{1}\right)$ and $p: M\left(p_{1}\right) \rightarrow B \times[0,1]$ be the obvious maps. Let $b \in B \subset M\left(p_{1}\right)$ and let $W$ be an open neighborhood of $b$ in $M\left(p_{1}\right)$. The only nontrivial point is to show that there exists a neighborhood $U$ of $b \times 1$ in $B \times[0,1]$ such that $p^{-1}(U) \subset W$.

Since $W$ is open in $M\left(p_{1}\right), \pi^{-1}(W) \subset E_{1} \times[0,1]$ is open and is a neighborhood of $\left(p_{1} \times \mathrm{id}\right)^{-1}(b \times 1)$. Since $p_{1} \times \mathrm{id}: E_{1} \times[0,1] \rightarrow B \times[0,1]$ is closed, $\left(p_{1} \times \mathrm{id}\right)\left(E_{1} \times[0,1]-\pi^{-1}(W)\right)$ is closed in $B \times[0,1]$ and does not contain $b \times 1$. Hence $B \times[0,1]-\left(p_{1} \times \mathrm{id}\right)\left(E_{1} \times[0,1]-\pi^{-1}(W)\right)$ is a neighborhood of $b \times 1$ : call it $U$. It is easy to check that $p^{-1}(U) \subset W$.

Definition 12.3. A map $p: E \rightarrow B$ is an approximate fibration if for every commuting diagram

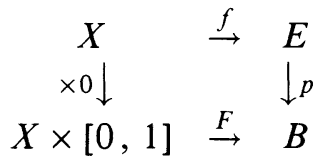

there is a controlled map $\tilde{F}: X \times[0,1] \times[0,1) \rightarrow E \times[0,1)$ from $F$ to $p$ such that $\tilde{F} \mid X \times\{0\} \times[0,1)=f \times \mathrm{id}_{[0,1)}$.

Before beginning, it is worth recording the following comments. If $p: E \rightarrow B$ is given then we can form $E(p)=\left\{(e, \lambda) \in E \times B^{I} \mid p(e)=\lambda(0)\right\}$. There are maps $\pi: E(p) \rightarrow E$ given by projection and $e v: E(p) \times I \rightarrow B$ given by $e v((e, \lambda) \times t)=\lambda(t)$. Hence we get a commutative square

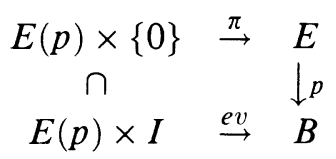

that we call the universal lifting problem.

The reason for the name comes from the fact that given any lifting problem

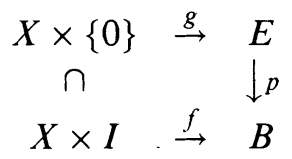

there is a map $F: X \rightarrow E(p)$ such that $g=\pi \circ F$ and $f=e v \circ F$. Furthermore, if $f(x, t)$ is a constant path for some $x \in X$, then $e v(F(x), t)$ is also a constant path. Hence, general lifting problems can be solved (even with regularity) iff the universal one can be. 
Notice that there is nothing in the definition of approximate fibration or fibration that requires the map $p$ to be onto and we do not assume that it is unless the contrary is stated. For fibrations this causes little concern since if $p: E \rightarrow B$ is a fibration then the image of $p$ in a path component of $B$ is either empty or the whole path component. Still, it is convenient to be able to talk about the associated Hurewicz fibration for any map and have it be a fibration.

The situation for approximate fibrations is slightly more bizarre. If $p: E \rightarrow B$ is an approximate fibration then the image of $p$ in any path component of $B$ is either empty or dense. In particular, the standard inclusion $(0,1) \rightarrow[0,1]$ is, for us, an approximate fibration. Notice that if $p$ is a closed map (which is often the case), then the image of $p$ is closed and hence is either empty or all of a particular path component.

We begin with some useful results on the behavior of approximate fibrations.

Lemma 12.4. Let $q: X \rightarrow B$ be a map, and let $p: E \rightarrow B$ be a fibration. Let $A \subset X$ be an NDR-pair. Suppose that we are given a controlled map $F^{c}: X \rightarrow$ $E$ such that $F^{c} \mid A$ is a constant family of fibre maps. Then $F^{c}$ is controlled homotopic to a constant family of fibre maps. The homotopy can be taken to be constant on $A$.

Remark. The resulting fibre map is well defined up to fibre homotopy equivalence, rel $A$. This follows formally from the relative version.

Proof. We begin by constructing a commutative square

$$
\begin{array}{ccc}
X \times[0,1) \times 0 & \stackrel{F^{c}}{\rightarrow} & E \\
\cap & & \downarrow \downarrow \\
X \times[0,1] \times[0,1] & \stackrel{g}{\rightarrow} & B
\end{array}
$$

where $g$ is defined as follows: $\bar{F}_{t}: X \times[0,1] \rightarrow B$ is clearly homotopic to (projection) $\circ\left(q \times \mathrm{id}_{[0,1]}\right)$, and this homotopy can be chosen to be rel $X \times 1$, where $\bar{F}: X \times[0,1] \rightarrow B$ is the extension of $p \circ F_{t}^{c}$ guaranteed in the definition of controlled map. Notice that if $F_{t}^{c} \mid A$ is a fibre map, $g$ can be chosen to be constant on $A \times[0,1] \times[0,1]$. Also notice that $g \mid X \times\{1\} \times[0,1] \rightarrow B$ is just $q$.

Since $p$ is a fibration, we can restrict $g$ to $X \times[0,1) \times[0,1]$ and then lift to get a map $G: X \times[0,1) \times[0,1] \rightarrow E$ which extends $F^{c}$ and which is constant on $A \times[0,1) \times[0,1]$. Notice that $G$ is a controlled homotopy between $F^{c}$ and $G(x, t, 1)$, and that $G(a, s, t)=F_{0}^{c}(a)$ for all $a \in A$.

Clearly $G(x, t, 1)$ is a fibre map for each $t$ in $[0,1)$, but perhaps not constant. It is now easy to find a homotopy of $G(x, t, 1)$ through fibre maps (and hence controlled) to the family that is constantly $G(x, 0,1)$. Moreover, this homotopy can be done rel $A$.

Lemma 12.5. Let $p: E \rightarrow B$ be a map, and let $i: E \rightarrow E(p)$ be the inclusion of $E$ into its associated Hurewicz fibration. Then $p$ is an approximate fibration iff 
there is a controlled map $R^{c}: E(p) \rightarrow E$ such that $R^{c} \circ i$ is controlled homotopic to $\mathrm{id}_{E}$. Moreover, if $R^{c}$ exists as above, then $i$ and $R^{c}$ are controlled homotopy equivalences with one map the inverse of the other.

Proof. To begin, suppose that $p$ is an approximate fibration and consider the following lifting problem

$$
\begin{array}{ccc}
E(p) \times 0 & \rightarrow & E \\
\cap & & \downarrow p \\
E(p) \times[0,1] & \stackrel{\text { evaluation }}{\rightarrow} & B
\end{array}
$$

We can find a controlled map $F^{c}: E(p) \times[0,1] \rightarrow E$, and we let $R^{c}=F^{c}(, 1)$. The composite $E \times[0,1] \rightarrow E(p) \times[0,1] \rightarrow E$ is a controlled homotopy between the identity on $E$ and $R^{c} \circ i$.

Conversely, suppose that an $R^{c}$ as above exists and that we are given a lifting problem

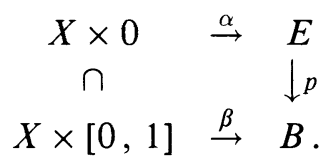

We can compose with $i$ to get a lifting problem for $E(p) \rightarrow B$, which of course we can solve, say by $L: X \times[0,1] \rightarrow E(p)$. Let $L^{c}: X \times[0,1] \rightarrow E(p) \rightarrow E$ be $L$ composed with $R^{c}$. It is a controlled map, but it probably does not extend the original map $\alpha$. Since $R^{c} \circ i$ is controlled homotopic to $\mathrm{id}_{E}$, there is a controlled homotopy $H^{c}: X \times[0,1] \rightarrow E$ which starts with $\alpha$ and finishes with $L^{c}(, 0)$. We can paste $L^{c}$ and $H^{c}$ together as follows.

Define $K^{c}: X \times[0,1] \rightarrow E$ by

$$
K_{t}^{c}(x, s)= \begin{cases}H^{c}\left(x, \frac{2 s}{1-t}\right) & \text { if } s \leq \frac{1-t}{2}, \\ L^{c}\left(x, \frac{2 s-1+t}{1+t}\right) & \text { if } s \geq \frac{1-t}{2},\end{cases}
$$

for $0 \leq t<1$. It is not hard to check that since $H^{c}$ is a controlled homotopy, the map $p \circ K^{c}$ completes using the map $\beta$, and hence is a solution to our lifting problem.

Lemma 12.6. Let $p: E \rightarrow B$ be an approximate fibration; let $q: X \rightarrow B$ be $a$ map; let $A \subset X$ be an NDR-pair; and let $F^{c}: A \rightarrow E$ be a controlled map. Then $F^{c}$ extends to a controlled map $X \rightarrow E$ iff the fibre map $A \rightarrow E(p)$ constructed in Lemma 12.4 from the controlled map $A \rightarrow E \rightarrow E(p)$ extends to a fibre map $X \rightarrow E(p)$.

Proof. Let $F^{c}$ extend to a controlled map $X \rightarrow E$. The process used in the proof of Lemma 12.4 is sufficiently natural to produce the required fibre extension.

Conversely, given the fibre extension, compose with the controlled map $R^{c}$ from Lemma 12.5. This gives a controlled map $X \rightarrow E$, but it probably does not extend our original controlled map on $A$. Nevertheless, the two controlled 
maps $A \rightarrow E$ are controlled homotopic. We can now use standard constructions with NDR-pairs to adjust our controlled map $X \rightarrow E$ by a controlled homotopy to a new controlled map which is our original one on $A$.

Here are some interesting corollaries. We omit the easy proofs.

Theorem 12.7 (Approximate Dold Theorem). A controlled map $F^{c}$ between two approximate fibrations for which the underlying map of spaces $F_{0}^{c}$ is a homotopy equivalence is a controlled homotopy equivalence.

Theorem 12.8 (Controlled Invariance of Approximate Fibrations). Given two spaces over $B$ which are controlled homotopy equivalent, one is an approximate fibration iff the other is.

Remark to the experts. This means that the notions of Hurewicz approximate fibration and Dold approximate fibration coincide.

Next we show that our definition agrees with the definition of Coram and Duvall under some restrictions.

Before beginning, we extend the notion of an approximate fibration. Let $p: E \rightarrow B$ be given, and suppose that we can solve the usual lifting problem, diagram $(* * *)$, for all spaces $X$ in a certain class. Then we say that $p$ is an approximate fibration for that class.

Definition 12.9. Let $p: E \rightarrow B$ be a map, $\mathscr{U}$ an open cover of $B$, and $\mathscr{C}$ a class of spaces. We say that $p$ has the $\mathscr{U}$-homotopy lifting property for $\mathscr{C}$ if, for every commuting diagram $(* * *)$ with $X \in \mathscr{C}$, there is a map $\tilde{F}: X \times[0,1] \rightarrow E$ such that $\tilde{F} \mid X \times\{0\}=f$ and $p \circ \tilde{F}$ is $\mathscr{U}$-close to $F$. We say that $p$ has the approximate homotopy lifting property for $\mathscr{C}$ if $p$ has the $\mathscr{U}$-homotopy lifting property for $\mathscr{C}$ for every open cover $\mathscr{U}$ of $B$. We say that $p$ has the approximate homotopy lifting property if $p$ has the approximate homotopy lifting property for the class of all spaces.

Coram and Duvall [8] originally defined a map to be an approximate fibration if it had the approximate homotopy lifting property. That definition seems to be inadequate when $B$ is not an ANR or when working categorically. However, we show below that the two definitions are equivalent with some mild restrictions on $E$ and $B$. Our definition is a mild extension of the one used by F. Quinn in [34], and Quinn knew his definition was equivalent to Coram and Duvall's under these conditions.

Lemma 12.10. If $p: E \rightarrow B$ is an approximate fibration for the class of paracompact spaces, then $p$ has the approximate homotopy lifting property for paracompact spaces.

Proof. Suppose we are given a lifting problem

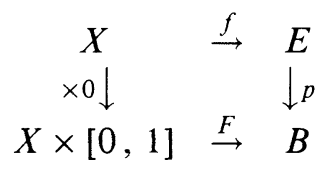


and an open cover $\mathscr{U}$ of $B$ with $X$ paracompact. By hypothesis there is a controlled map $\tilde{F}: X \times[0,1] \times[0,1) \rightarrow E \times[0,1)$ from $F$ to $p$ such that $\tilde{F} \mid X \times\{0\} \times[0,1)=f \times \mathrm{id}_{[0,1)}$. We also have a map $\widehat{F}: X \times[0,1] \times[0,1] \rightarrow B \times$ $[0,1]$ defined by $\widehat{F} \mid X \times[0,1] \times[0,1)=(p \times$ id $) \tilde{F}$ and $\widehat{F} \mid X \times[0,1] \times\{1\}=F$.

For each $x$ in $X \times[0,1]$ choose an open subset $V_{x}$ of $X \times[0,1]$ containing $x$ and a real number $r_{x}$ in $[0,1)$ such that $\widehat{F}\left(V_{x} \times\left(r_{x}, 1\right]\right)$ is contained in (some member of $\mathscr{U}) \times[0,1]$. By paracompactness of $X \times[0,1]$ there exists a locally (neighborhood)-finite open cover $\mathscr{V}$ refining $\left\{V_{x} \mid x \in X\right\}$ covering $X \times[0,1]$. For each $V$ in $\mathscr{V}$ choose an $x(V)$ in $X \times[0,1]$ such that $V \subset V_{x(V)}$.

Again using the paracompactness of $X \times[0,1],[11$, p. 179], there exists a $\operatorname{map} \alpha: X \times[0,1] \rightarrow[0,1)$ such that $\alpha(x) \geq \min \left\{r_{x(V)} \mid x \in V\right\}$ for each $x$ in $X \times[0,1]$. Define $\tilde{\alpha}: X \times[0,1] \rightarrow X \times[0,1] \times[0,1)$ by setting $\tilde{\alpha}(x)=$ $(x, \alpha(x))$. Then the composition $X \times[0,1] \stackrel{\tilde{\alpha}}{\rightarrow} X \times[0,1] \times[0,1) \stackrel{\tilde{F}}{\rightarrow} E \times$ $[0,1) \stackrel{\text { proj }}{\rightarrow} E$ is an approximate $\mathscr{U}$-lift of $F$.

Lemma 12.11. If $B$ is an $A N R$ (an absolute neighborhood retract for the class of metric spaces) and $p: E \rightarrow B$ is a map, and if $p$ has the approximate homotopy lifting property, then $p$ is an approximate fibration.

In the proof to follow, we need a version of Theorem 1.2 of $\mathrm{Hu}[16, \mathrm{p} .112]$, which is slightly different than the stated one. The theorem says that close maps from a metric space $X$ into an ANR are closely homotopic, and if we already have a close homotopy on a closed subset $A$, we can extend the homotopy. We need a version in which $X$ is arbitrary but $A$ is an NDR subspace of $X$. We indicate the needed changes in the proof (notation as in [16]). Nothing need be changed until the top of page 113, where we wish to extend a map defined on $Q$ to a neighborhood of $Q$ in $P$. Since $(X, A)$ is an NDR-pair, so is $(P, Q)$ so the extension presents no difficulty. If we let $u: X \rightarrow[0,1]$ be the function which displays $(X, A)$ as an NDR-pair, then we easily see that for the $C$ we need to choose in the middle of page 113 we may take $u^{-1}([0,1 / 2))$. It is now no trouble to select the set $B$ and function $e$ as required and finish the proof.

Proof of 12.11. First note that since $B$ is metric, $p$ has the regular approximate homotopy lifting property [7, p. 39]. Suppose we are given our usual lifting problem

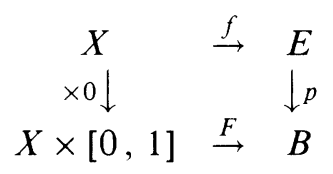

We need to define a controlled map $\tilde{F}: X \times[0,1] \times[0,1) \rightarrow E \times[0,1)$ from $F$ to $p$ such that $\tilde{F} \mid X \times\{0\} \times[0,1)=f \times \mathrm{id}_{[0,1)}$.

Choose a sequence, $\left\{\varepsilon_{i}\right\}_{i=1}^{\infty}$ of positive numbers converging to 0 . Since $B$ is an ANR, we can find open covers $\delta_{i}, i=1,2, \ldots$, of $B$ so that any two maps into $B$ which are $\delta_{i}$-close are $\varepsilon_{i}$-homotopic (rel any NDR subset where 
they agree). We also assume that the diameter of any member of $\delta_{i}$ is less than $\varepsilon_{i-1}$ (if $i>1$ ).

We will inductively define maps $\tilde{F}_{i}: X \times[0,1] \times[0,1-1 / i] \rightarrow E \times[0,1-1 / i]$ for $i=1,2, \ldots$ satisfying the following properties:

(1) $\tilde{F}_{i}$ is an extension of $\tilde{F}_{i-1}$ for $i>1$,

(2) $\tilde{F}_{i}$ is fibre-preserving over $[0,1-1 / i]$,

(3) $\tilde{F}_{i} \mid X \times\{0\} \times[0,1-1 / i]=f \times$ id,

(4) $p \circ \tilde{F}_{i} \mid X \times[0,1] \times\{1-1 / i\}$ is $\delta_{i}$-close to $F$,

(5) $p \circ \operatorname{proj}_{E} \circ \tilde{F}_{i} \mid X \times[0,1] \times[1-1 /(i-1), 1-1 / i]$ is $2 \varepsilon_{i}$-close to $F \times$ id for $i>1$.

To begin the inductive construction, let $\tilde{F}_{1} \mid X \times[0,1] \times\{0\} \rightarrow E \times\{0\}$ be a map such that $\tilde{F}_{1} \mid X \times\{0\} \times\{0\}=f$ and $p \circ \tilde{F}_{1}$ is $\delta_{1}$-close to $F$. Inductively assume that $i \geq 1$ and $\tilde{F}_{i}$ has already been defined. Since $p \circ$ $\tilde{F}_{i} \mid X \times[0,1] \times\{1-1 / i\}$ is $\delta_{i}$-close to $F$, there is an $\varepsilon_{i}$-homotopy between $p \circ \tilde{F}_{i} \mid$ and $F$ rel $X \times\{0\} \times\{1-1 / i\}$.

Let $\tilde{F}_{i+1} \mid X \times[0,1] \times[1-1 / i, 1-1 /(i+1)] \rightarrow E \times[1-1 / i, 1-1 /(i+1)]$ be a $\delta_{i+1}$-lift of this homotopy. By the regular lifting property mentioned above, we can assume that $\tilde{F}_{i+1} \mid X \times\{0\} \times[1-1 / i, 1-1 /(i+1)]=f \times$ id. $\tilde{F}$.

It is clear that the $\tilde{F}$ 's piece together to define the required controlled map

Lemmas 12.10 and 12.11 say roughly that there is little difference between our definition of an approximate fibration and that of Coram and Duvall. In the next few results we explore some of the Coram and Duvall results, taking some care to avoid spurious point set assumptions. The first few results culminate in the result that Serre approximate fibrations are often approximate fibrations. We begin with

Lemma 12.12. Let $p: E \rightarrow B$ be a map which has the approximate homotopy lifting property for cells. Suppose that $B$ is paracompact. Then $p$ has the relative approximate homotopy lifting property for pairs $(X, A)$ with $X$ an $A N R$ and $A$ a closed ANR subspace.

Proof. The relative approximate homotopy lifting property for pairs $(X, A)$ means that given a commutative square

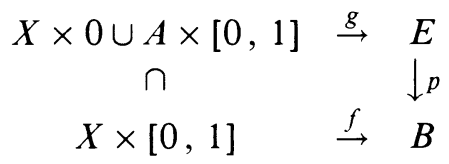

and a cover $\varepsilon$ of $B$, we can find a map $F: X \times[0,1] \rightarrow E$ extending $g$ such that $f$ and $p \circ F$ are $\varepsilon$-close.

A standard "fold" argument shows that if $p$ has the approximate homotopy lifting property for cells, then $p$ has the relative approximate homotopy lifting property for a cell rel its boundary. 
We also need the $\varepsilon$-homotopy extension theorem. This says that if $X$ is normal; $(X, A)$ is an NDR-pair; $f: X \rightarrow B$ is a map, where $B$ is paracompact; and $F: A \times[0,1] \rightarrow B$ is an $\varepsilon$-homotopy of $f \mid A$, where $\varepsilon$ is a cover of $B$, then $F$ has an extension to $G: X \times[0,1] \rightarrow B$ where $G$ is an $\varepsilon$-homotopy. The proof is routine.

We begin our proof by proving the relative approximate homotopy extension theorem holds for $\mathrm{CW}$ pairs, $(K, L)$. Let $K^{i}$ denote $L$ union the $i$-skeleton of $K ; K^{-1}=L$. We construct the needed map on the skeleta. For $K^{-1}$ there is nothing to do.

First choose a cover $\delta$ of $B$ so that $\delta$-close maps are $\varepsilon$-homotopic. Next choose a cover, denoted $\delta^{\prime}$, so that $\delta^{\prime}$-close maps are $\delta / 2$-homotopic, where $\delta / 2$ is a double star, locally finite, refinement of $\delta$. For $K^{0}$, use the approximate homotopy lifting property for cells to get a map $F_{1}: K^{0} \times[0,1] \rightarrow E$ which is $g$ on $L \times[0,1]$ and such that $p \circ F_{1}$ is $\delta^{\prime}$-close to $f$. Use the $\varepsilon$-homotopy extension theorem to get a $\delta / 2$-homotopy to $f_{1}: K \times[0,1] \rightarrow B$ rel $K^{-1} \times[0,1]$ where $f_{1}=p \circ F_{1}$.

Next choose a cover, say $\delta^{\prime \prime}$, so that $\delta^{\prime \prime}$-close maps are $\delta / 4$-homotopic, where $\delta / 4$ is a double star, locally finite refinement of $\delta^{\prime}$. Use the approximate homotopy lifting property for cells to get a map $F_{2}: K^{1} \times[0,1] \rightarrow E$ which is $F_{1}$ on $K^{0} \times[0,1]$ and such that $p \circ F_{2}$ is $\delta^{\prime \prime}$-close to $f_{1}$. Use the $\varepsilon$-homotopy extension theorem to get a $\delta / 4$-homotopy of $f_{1}$ to $f_{2}: K \times[0,1] \rightarrow B$ rel $K^{0} \times[0,1]$.

Continue in this vein to construct $F_{i+1}: K^{i} \times[0,1] \rightarrow E$ such that $F_{i+1} \mid K^{i-1} \times$ $[0,1]=F_{i}$; and $f_{i+1}: K \times[0,1] \rightarrow B$ so that $p \circ f_{i+1}$ is $\delta / 2^{i+1}$-homotopic to $p \circ f_{i}$ rel $K^{i-1} \times[0,1]$, where $\delta / 2^{i+1}$ is a twice star refinement of $\delta / 2^{i}$. The $F_{i}$ piece together to give a map $G: K \times[0,1] \rightarrow E$ which extends $g$ on $L \times[0,1]$, and we need to see that $p \circ G$ is $\varepsilon$-close to $f$. Fix a point $(x, t) \in K \times[0,1]$. If $(x, t) \in L \times[0,1]$, then $p \circ G(x, t)=p \circ g(x, t)=f(x, t)$ so they are close. If $(x, t) \notin L \times[0,1]$, then there is some $i \geq 0$ such that $(x, t) \in K^{i} \times[0,1]$. But then $p \circ G(x, t)=p \circ F_{i+1}(x, t)$ and $f(x, t)$ are $\delta / 2+\delta / 4+\cdots+\delta / 2^{i+1}<\delta$ close, and hence $\varepsilon$-close. (The numerology in the last line is actually correct.)

If $(X, A)$ is a pair of ANR's, and $\varepsilon$ is a cover of $X$, then we can find a pair of CW complexes $(K, L)$ and maps of pairs $(X, A) \rightarrow(K, L)$ and $(K, L) \rightarrow(X, A)$ so that the composite $(X, A) \rightarrow(K, L) \rightarrow(X, A)$ is $\varepsilon$ homotopic to the identity, as a map of pairs. Now we use the Coram-Duvall trick [9, Lemma 2.5, p. 45$]$ to get the required maps.

Lemma 12.12 allows us to prove

Theorem 12.13. Let $p: E \rightarrow B$ be a map between ANR's. If $p$ has the approximate homotopy lifting property for cells then $p$ is an approximate fibration.

Remark. The converse holds by Lemma 12.10 without any point set conditions on $E$ and $B$. Some conditions on the total space and base are necessary since 
natural projection from the cone on the Warsaw circle to $[0,1]$ is a Serre fibration that is not an approximate fibration.

Proof. We can finish by constructing bridge functions just as Coram and Duvall do, [9, Proof of 2.2, p. 45]. Alternatively we remark that it is enough to solve the universal lifting problem. By Lemma 12.12 we can do this if $E(p)$ is an ANR. This is just Lemma 4.6 on page 246 of Allaud and Fadell [1]. The only caveat is that they mean separable ANR when they write ANR, so the careful reader needs to check that they really do not need separable. Any ANR is ULC by Dugundji [12] so the proof proceeds with no modifications.

One use for Theorem 12.13 is

Corollary 12.14. If $p: E \rightarrow B$ is an approximate fibration with $E$ and $B$ ANR's, and if $U \subset B$ is open, then $p \mid: p^{-1}(U) \rightarrow U$ is an approximate fibration.

Proof. Even if we only assume that $B$ is Hausdorff, $p \mid: p^{-1}(U) \rightarrow U$ has the approximate homotopy lifting property for cells. Both $U$ and $p^{-1}(U)$ are open subsets of ANR's, and hence ANR's. Then Theorem 12.13 shows that $p \mid: p^{-1}(U) \rightarrow U$ is an approximate fibration.

Next, we record a consequence of the $k$-movability criterion of Coram and Duvall first noticed by $\mathrm{F}$. Quinn. Given any map of spaces, say $p: E \rightarrow B$, we can form the homotopy fibre of $p$ over any point $b \in B$. It is just the honest fibre over $b$ of the associated Hurewicz fibration $E(p) \rightarrow B$. Given two maps $p_{0}: E_{0} \rightarrow B_{0}$ and $p_{1}: E_{1} \rightarrow B_{1}$ and maps $g: E_{0} \rightarrow E_{1}$ and $h: B_{0} \rightarrow B_{1}$ such that $p_{1} \circ g=h \circ p_{1}$, then there is a map from the homotopy fibre of $p_{0}$ over $b_{0} \in B_{0}$ to the homotopy fibre of $p_{1}$ over $h\left(b_{0}\right)$.

Theorem 12.15. Let $p: E \rightarrow B$ be given with $B$ an ANR. Let $\beta$ be a basis for $B$. Suppose that for every $U \in \beta$ the map from the homotopy fibre of $p \mid: p^{-1}(U) \rightarrow$ $U$ to the homotopy fibre of $p$ induces an isomorphism on homotopy groups for all $b \in U$. Then $p$ has the approximate homotopy lifting property for cells. The converse is true even if $B$ is merely paracompact.

Remark. It is no harder to first extend the definition of $k$-movability due to Coram and Duvall to our case. We say that a map $p: E \rightarrow B$ is $k$-movable provided it has the following property. For each $b \in B$ such that $p^{-1}(b)$ is nonempty, form $\lim _{\leftarrow} \pi_{j}\left(p^{-1}(U), e\right)$ for a fixed $e \in p^{-1}(b)$. We say that $p$ is $k$-movable provided given any point $c \in B$ and neighborhood $U$ of $c$, there exist neighborhoods $V \subset W \subset U$ of $c$ and a point $b \in V$ with $p^{-1}(b)$ nonempty, such that:

the image of the above inverse limit in $\pi_{j}\left(p^{-1}(W), e\right)$ is equal to the image of $\pi_{j}\left(p^{-1}(V), e\right)$ in $\pi_{j}\left(p^{-1}(W), e\right)$ for all $e \in$ $p^{-1}(b)$ and for all $0 \leq j \leq k$.

Note that if $p$ is proper and $B$ is compactly-generated, then our definition of $k$-movable coincides with that of Coram and Duvall. 
Proof. One representation for the $k$ th homotopy group of the fibre is as maps $\alpha: S^{k-1} \rightarrow E$ and $\gamma: D^{k} \rightarrow B$ such that $p \circ \alpha=\gamma \mid$ modulo the appropriate sort of homotopies. To show the converse part of the theorem is just a diligent application of approximate homotopy lifting of finite complexes rel subcomplexes.

To prove Theorem 12.15 it will suffice to prove

Lemma 12.16. If $p: E \rightarrow B$ is k-movable, $B$ an $A N R$, then $p$ has the approximate homotopy lifting property for cells of dimension $\leq k$.

The reason that Lemma 12.16 suffices is that any map satisfying the hypotheses of Theorem 12.15 is clearly $k$-movable for all $k$. (This uses $B$ is locally-contractible.)

The proof we present here is cribbed from Coram and Duvall [9, Theorem 3.3, p. 49]. To begin, we need to adjust Lemma 3.1 on p. 47 . With our definition of $k$-movable, the lemma reads just as in Coram and Duvall except that the arbitrary neighborhoods $U_{0}$ and $W_{0}$ are restricted to be of the form $p^{-1}$ (of an open set in $B$ ) and the neighborhoods $U, V, W$ and $Z$ constructed in the lemma are also of this form.

We now follow Coram and Duvall's proof of Theorem 3.3. We take just a little care to choose our sets down in $B$ rather than up in $E$ as they do. The proof proceeds easily until the next to the last line where Lemma 3.2 is invoked. The reader who has followed the proof to this point will see that it is Lemma 3.1 that is required and the necessary version of that we have.

The following proposition is not needed for our results, but it shows that two potentially different definitions of a $k$-simplex in $M A F(B)$ are really the same.

Proposition 12.17. Let $E$ and $B$ denote locally compact, separable metric $A N R$ 's. If $p: E \times \Delta^{k} \rightarrow B \times \Delta^{k}$ is a proper fibre-preserving map over $\Delta^{k}$, then $p$ is an approximate fibration iff $p_{t}: E \times\{t\} \rightarrow B \times\{t\}$ is an approximate fibration for every $t \in \Delta^{k}$.

Proof. If $p$ is an approximate fibration, then each $p_{t}$ is because $E \times\{t\}$ is a deformation retract of $E \times \Delta^{k}$ over a deformation retraction of $B \times \Delta^{k}$ into $B \times\{t\}$.

Conversely, if each $p_{t}$ is an approximate fibration, then it follows from [18, Theorem 2.4], that $p$ has a certain "sliced" approximate homotopy lifting property. Using that property, one can see that $p$ is completely movable in the sense of [9]. The result now follows from [9].

\section{SUCKING FOR MANIFOLD APPROXIMATE FIBRATIONS}

If $\beta$ is an open cover of $B$ and $p: E \rightarrow B$ is a map, then we say that $p$ is a $\beta$-fibration if $p$ has the $\beta$-homotopy lifting property. We record here results which say, that under appropriate conditions, $\beta$-fibrations can be deformed to nearby approximate fibrations. Thus, approximate fibrations "suck". Chapman 
[6] had the first results along these lines. The parameterized versions are due to Hughes [20].

Theorem 13.1. Let $B$ be a manifold, let $m \geq 5, k \geq 0$, and let $\alpha$ be an open cover of $B$. There exists an open cover $\beta$ of $B$ so that if $M$ is an m-manifold without boundary and $f: M \times \Delta^{k} \rightarrow B \times \Delta^{k}$ is a proper map which preserves the fibres over $\Delta^{k}$ such that $f_{t}: M \rightarrow B$ is a $\beta$-fibration for each $t \in \Delta^{k}$ and an approximate fibration for each $t \in \partial \Delta^{k}$, then there is a fibre preserving (over $\Delta^{k}$ ) approximate fibration $\tilde{f}: M \times \Delta^{k} \rightarrow B \times \Delta^{k}$ such that $\tilde{f}_{t}$ is $\alpha$-close to $f_{t}$ for each $t$ in $\Delta^{k}$ and $\tilde{f}\left|M \times \partial \Delta^{k}=f\right| M \times \partial \Delta^{k}$.

Remarks on the proof. For the case $k=0$ see Chapman [6]. For $k \geq 1$, see Hughes [20]. However, note that in [20] it is assumed that $B$ has a handlebody decomposition or is a polyhedron. It is fairly routine to make the arguments of [20] work more generally. Here are the details.

One uses the handle lemmas in [20] to prove the following lemma from which the result follows.

Lemma 13.2. Let $B$ be a manifold, let $m \geq 5$, and let $k \geq 0$. Let $C$ and $\tilde{C}$ be compact subsets of some chart of $B$ such that $C \subset \operatorname{int} \tilde{C}$. Let $D$ and $\tilde{D}$ be closed subsets of $B$ with $D \subset$ int $\tilde{D}$. For every open cover $\alpha$ of $B$ there exists an open cover $\beta$ such that: for every open cover $\mu$ there exists an open cover $v$ so that if $M$ is an m-manifold without boundary and $f: M \times \Delta^{k} \rightarrow B \times \Delta^{k}$ is a fibre-preserving (over $\Delta^{k}$ ) map such that $f_{t}: M \rightarrow B$ is a $\beta$-fibration and a v-fibration over $\tilde{D}$ for each $t$ in $\Delta^{k}$ and an approximate fibration for each $t$ in $\partial \Delta^{k}$, then there exists a fibre-preserving map $\tilde{f}: M \times \Delta^{k} \rightarrow B \times \Delta^{k}$ such that $\tilde{f}_{t}$ is a $\mu$-fibration over $C \cup D$ for each $t$ in $\Delta^{k}$ and $\tilde{f}$ is fibre-preserving $\alpha$-homotopic to $\left.f \operatorname{rel}\left(\left(M \times \Delta^{k}\right)-f^{-1}(\tilde{C}-D) \times \Delta^{k}\right)\right) \cup\left(M \times \partial \Delta^{k}\right)$.

We also need the following version of 13.1 for manifolds with boundary. For an indication of proof, see Chapman [6].

Addendum 13.3. If, in Theorem 13.1, the manifold $M$ is allowed to have a boundary $\partial M$ and we require that each $f_{t}$ already be an approximate fibration over an open subset of $B$ containing $f_{t}(\partial M)$, then we can additionally require $\tilde{f}$ to agree with $f$ on $\partial M \times \Delta^{k}$.

\section{Straightening}

A key property is that parameterized families of approximate fibrations can be "straightened"; that is, isotoped to be nearly a product family. We begin by quoting an $\alpha$ version of this due to Hughes [20] and then derive a "controlled" version from the $\alpha$ version and sucking.

Theorem 14.1 ( $\alpha$ Straightening). Let $M$ and $B$ be manifolds, $\partial M=\varnothing$, dimension $M \geq 5$, and let $p: M \times \Delta^{k} \times[0,1] \rightarrow B \times \Delta^{k} \times[0,1]$ be a proper 
fibre-preserving over $\Delta^{k} \times[0,1]$ map such that $p_{t}: M \rightarrow B$ is an approximate fibration for each $t$ in $\Delta^{k} \times[0,1]$. For every open cover $\alpha$ of $B$ there exists a fibre-preserving homeomorphism $H: M \times \Delta^{k} \times[0,1] \rightarrow M \times \Delta^{k} \times[0,1]$ such that $H \mid M \times \Delta^{k} \times\{0\}=\mathrm{id}$ and $p \circ H$ is $\alpha$-close to $p \mid\left(M \times \Delta^{k} \times\{0\}\right) \times \mathrm{id}_{[0,1]}$. Moreover, if $p \mid M \times \partial \Delta^{k} \times[0,1]=\left(p \mid M \times \partial \Delta^{k} \times\{0\}\right) \times \mathrm{id}_{[0,1]}$, then we can find $H$ so that $H \mid M \times \partial \Delta^{k} \times[0,1]=\mathrm{id}$.

We also need the following version for manifolds with boundary. The proof follows from the proof of 14.1 given in [20].

Addendum 14.2. Let $B$ be a manifold, $U$ an open subset of $B, m \geq 5$, and $n \geq 0$. For every open cover $\alpha$ of $B$ there exists an open cover $\beta$ of $B$ such that if $M$ is an m-manifold with boundary $\partial M, p: M \times \Delta^{k} \times[0,1] \rightarrow B \times \Delta^{k} \times[0,1]$ is a proper fibre preserving map over $\Delta^{k} \times[0,1]$ such that

$p_{t}: M \rightarrow B$ is an approximate fibration for $t$ in $\Delta^{k} \times[0,1]$;

$\partial M \times \Delta^{k} \times[0,1] \subset p^{-1}\left(U \times \Delta^{k} \times[0,1]\right) ;$ and

$G: p^{-1}\left(U \times \Delta^{k} \times[0,1]\right) \rightarrow M \times \Delta^{k} \times[0,1]$ is a fibre-preserving open embedding so that $G \mid p^{-1}\left(U \times \Delta^{k} \times\{0\}\right)=$ id and $p \circ G$ is $\beta$-close to $p \mid p^{-1}\left(U \times \Delta^{k} \times\{0\}\right) \times \mathrm{id}_{[0,1]}$,

then there exists a fibre preserving homeomorphism $H: M \times \Delta^{k} \times[0,1] \rightarrow$ $M \times \Delta^{k} \times[0,1]$ such that $H \mid M \times \Delta^{k} \times\{0\}=$ id and $p \circ H$ is $\alpha$-close to $p \mid\left(M \times \Delta^{k} \times\{0\} \times \mathrm{id}_{[0,1]}\right.$, and $H=G$ in a neighborhood of $\partial M \times \Delta^{k} \times[0,1]$.

Theorem 14.3 (Controlled Straightening). Let $M$ and $B$ be manifolds, $\partial M=$ $\varnothing$, dimension $M \geq 5$, and let $p: M \times \Delta^{k} \times[0,1] \rightarrow B \times \Delta^{k} \times[0,1]$ be a proper fibre-preserving map over $\Delta^{k} \times[0,1]$ such that $p_{t}: M \rightarrow B$ is an approximate fibration for $t$ in $\Delta^{k} \times[0,1]$. Then there exists a homeomorphism $H: M \times \Delta^{k} \times$ $[0, \infty) \rightarrow M \times \Delta^{k} \times[0, \infty)$ such that

(i) $H \mid M \times \Delta^{k} \times\{0\}=$ id,

(ii) $H$ is fibre-preserving over $\Delta^{k} \times[0, \infty)$,

(iii) $\left(p_{1} \times \mathrm{id}_{[0, \infty)}\right) H: M \times \Delta^{k} \times[0, \infty) \rightarrow B \times \Delta^{k} \times[0, \infty)$ extends to a map $M \times \Delta^{k} \times[0, \infty] \rightarrow B \times \Delta^{k} \times[0, \infty]$ via $p_{0}$, where $p_{i}=p \mid M \times \Delta^{k} \times\{i\}$ : i.e. $H$ is a controlled homeomorphism from $p_{0}$ to $p_{1}$.

Moreover, if $p \mid M \times \partial \Delta^{k} \times[0,1]=\left(p \mid M \times \partial \Delta^{k} \times\{0\}\right) \times \mathrm{id}_{[0,1]}$, then we can find $H$ so that $H \mid M \times \partial \Delta^{k} \times[0, \infty)=\mathrm{id}$.

Proof. The technique used in this proof comes from Hughes [21]. Choose a sequence $\left\{\varepsilon_{i}\right\}_{i=0}^{\infty}$ of positive numbers converging to 0 . For each $i=0,1,2, \ldots$ let $\delta_{i}$ be an open cover of $B$ such that any two $k$-parameter families of approximate fibrations from $M$ to $B$ which are $\delta_{i}$-close are $\varepsilon_{i}$-homotopic through $k$-parameter families of approximate fibrations (Hughes [20]). Assume that the diameter of each member of $\delta_{i}$ is less than $\varepsilon_{i}$. 
For each $i=0,1,2, \ldots$ we will construct a homeomorphism $h^{i}: M \times \Delta^{k} \times$ $[i, i+1] \rightarrow M \times \Delta^{k} \times[i, i+1]$ such that

(1) $h^{i}$ is fibre-preserving over $\Delta^{k} \times[i, i+1]$,

(2) $h^{0} \mid M \times \Delta^{k} \times\{0\}=$ id and if $i>0, h^{i}\left|M \times \Delta^{k} \times\{i\}=h^{i-1}\right|$,

(3) $\left(p_{1} \times\right.$ id $) \circ h^{i}$ is $\left(\varepsilon_{i}+\delta_{i+1}\right)$-close to $p_{0} \times$ id if $i>0$,

(4) $p_{1} \circ h^{i} \mid M \times \Delta^{k} \times\{i+1\}$ is $\delta_{i+1}$-close to $p_{0}$.

It is clear that once the $h^{i}$ are constructed, they piece together to define $H$.

The existence of $h^{0}: M \times \Delta^{k} \times[0,1] \rightarrow M \times \Delta^{k} \times[0,1]$ follows directly from 14.1. Assume that $i>0$ and that $h^{i-1}$ is already defined. Since $p_{1} \circ$ $h^{i-1} \mid M \times \Delta^{k} \times\{i\}$ is $\delta_{i}$-close to $p_{0}$, there exists a fibre-preserving map over $\Delta^{k} \times[0,1], g^{i}: M \times \Delta^{k} \times[0,1] \rightarrow B \times \Delta^{k} \times[0,1]$ such that $g^{i} \mid M \times \Delta^{k} \times\{0\}=p_{0}$, $g^{i}\left|M \times \Delta^{k} \times\{1\}=p_{1} \circ h^{i-1}\right| M \times \Delta^{k} \times\{i\}, g^{i}$ is an $\varepsilon_{i}$-homotopy, and $g_{t}^{i}: M \rightarrow B$ is an approximate fibration for each $t$ in $\Delta^{k} \times[0,1]$.

Use 14.1 on $g^{i}$ to find a homeomorphism $k^{i}: M \times \Delta^{k} \times[0,1] \rightarrow M \times \Delta^{k} \times$ $[0,1]$ such that $k^{i}$ is fibre-preserving over $\Delta^{k} \times[0,1] ; k^{i} \mid M \times \Delta^{k} \times\{0\}=\mathrm{id}$, and $g^{i} \circ k^{i}$ is $\delta_{i+1}$-close to $\left(g^{i} \mid M \times \Delta^{k} \times\{0\}\right) \times \mathrm{id}=p_{0} \times \mathrm{id}$.

Then define $h^{i}: M \times \Delta^{k} \times[i, i+1] \rightarrow M \times \Delta^{k} \times[i, i+1]$ by setting $h_{t}^{i}=$ $h_{i}^{i-1} \circ k_{t-i}^{i}$ for each $t$ in $\Delta^{k} \times[i, i+1]$.

If in addition, we are given that $p$ is straight on $M \times \partial \Delta^{k} \times[0,1]$, then it is easy to use the full strength of the results quoted above to construct $H$ so that $H \mid M \times \partial \Delta^{k} \times[0, \infty)=\mathrm{id}$.

The following version of straightening is used to verify the straightening and strong straightening properties for $M A F$ as stated in $\S 7$.

Corollary 14.4. Suppose given the hypotheses of Theorem 14.3. Let $p_{0}=p \mid: M \times$ $\Delta^{k} \times\{0\} \rightarrow B \times \Delta^{k} \times\{0\}$. Then there exists a homeomorphism $H$ from $M \times$ $\Delta^{k} \times[0,1] \times[0,1)$ to itself such that

(i) $H=$ id on $M \times \Delta^{k} \times[0,1] \times\{0\}$ and $M \times \Delta^{k} \times\{0\} \times[0,1)$,

(ii) $H$ is fibre-preserving over $\Delta^{k} \times[0,1] \times[0,1)$,

(iii) $H$ is a controlled homeomorphism from $p$ to $p_{0} \times \mathrm{id}_{[0,1]}$.

Moreover, if $p \mid M \times \partial \Delta^{k} \times[0,1]=\left(p_{0} \mid M \times \partial \Delta^{k}\right) \times \mathrm{id}_{[0,1]}$, then we can find $H$ so that $H \mid M \times \partial \Delta^{k} \times[0,1] \times[0,1)=\mathrm{id}$.

Proof. It is easy to construct a map $q: M \times \Delta^{k} \times[0,1]^{2} \rightarrow M \times \Delta^{k} \times[0,1]^{2}$ which is fibre-preserving over $\Delta^{k} \times[0,1]^{2}$ and each slice $q_{t}: M \rightarrow B$ is an approximate fibration such that

$$
\begin{aligned}
& q \mid M \times \Delta^{k} \times[0,1] \times\{0\}=p, \\
& q \mid M \times \Delta^{k} \times[0,1] \times\{1\}=p_{0} \times \mathrm{id}_{[0,1]}, \text { and } \\
& q \mid M \times \Delta^{k} \times\{0\} \times[0,1]=p_{0} \times \mathrm{id}_{[0,1]} .
\end{aligned}
$$


Apply the straightening principles of Theorem 14.3 to the map $q$.

Of course there is a version of 14.3 for manifolds with boundary with hypotheses similar to those of 14.2.

Corollary 14.5. Let $B$ be a manifold and let $p: E \rightarrow B \times[0,1]$ be a proper map such that

(i) the composition $\pi: E \stackrel{p}{\rightarrow} B \times[0,1] \stackrel{\text { proj }}{\rightarrow}[0,1]$ is a fibre bundle projection with fibre $F$ which is a manifold of dimension $\geq 5$ such that $\partial F=\varnothing$,

(ii) $p \mid: \pi^{-1}(t) \rightarrow B \times\{t\}$ is a proper approximate fibration for each $t$ in $[0,1]$.

Then $p \mid: \pi^{-1}(0) \rightarrow B \times\{0\}$ is controlled homeomorphic to $p \mid: \pi^{-1}(1) \rightarrow B \times\{1\}$.

Proof. Let $k: \pi^{-1}(0) \times[0,1] \rightarrow E$ be a trivializing homeomorphism for $p$ such that $k_{0}=k \mid: \pi^{-1}(0) \times\{0\} \rightarrow \pi^{-1}(0)$ is the identity. Then $p \circ k: \pi^{-1}(0) \times$ $[0,1] \rightarrow B \times[0,1]$ is a family of approximate fibrations to which Theorem 14.3 applies (with $k=0$ ). Let $H: \pi^{-1}(0) \times[0, \infty) \rightarrow \pi^{-1}(0) \times[0, \infty)$ be the homeomorphism provided by Theorem 14.3. In particular, $\left(p_{1} \circ k_{1} \times \mathrm{id}\right) \circ H$ extends via $p_{0} \circ k_{0}=p_{0}$. Thus, $\left(k_{1} \times \mathrm{id}\right) \circ H$ is a controlled homeomorphism from $p_{0}$ to $p_{1}$.

As promised in $\S 1 . D$, we now show that the notion of the fibre germ of a manifold approximate fibration is well-defined. For notation, let $r: \mathbf{R}^{i} \rightarrow \mathbf{R}^{i}$ be defined by $r\left(x_{1}, x_{2}, \ldots, x_{i}\right)=\left(-x_{1}, x_{2}, \ldots, x_{i}\right)$.

Corollary 14.6. Let $B$ be a connected $i$-manifold, let $p: M \rightarrow B$ be a manifold approximate fibration such that dimension $M \geq 5$ and $\partial M \subset p^{-1}(\partial B)$. Let $g_{k}: \mathbf{R}^{i} \rightarrow B, k=0,1$, be two open embeddings. Then $p \mid: p^{-1}\left(g_{0}\left(\mathbf{R}^{i}\right)\right) \rightarrow g_{0}\left(\mathbf{R}^{i}\right)$ is controlled homeomorphic to either $p \mid: p^{-1}\left(g_{1}\left(\mathbf{R}^{i}\right)\right) \rightarrow g_{1}\left(\mathbf{R}^{i}\right)$ or $p \mid: p^{-1}\left(g_{1}\left(\mathbf{R}^{i}\right)\right)$ $\rightarrow g_{1} \circ r\left(\mathbf{R}^{i}\right)$. If $B$ is nonorientable then $p \mid: p^{-1}\left(g_{0}\left(\mathbf{R}^{i}\right)\right) \rightarrow g_{0}\left(\mathbf{R}^{i}\right)$ is controlled homeomorphic to both $p \mid: p^{-1}\left(g_{1}\left(\mathbf{R}^{i}\right)\right) \rightarrow g_{1}\left(\mathbf{R}^{i}\right)$ and $p \mid: p^{-1}\left(g_{1}\left(\mathbf{R}^{i}\right)\right) \rightarrow$ $g_{1} \circ r\left(\mathbf{R}^{i}\right)$.

Proof. It follows from the truth of the annulus conjecture that $g_{0}$ is isotopic to either $g_{1}$ or $g_{1} \circ r$ [26 and 33]. Let $h_{t}: \mathbf{R}^{i} \rightarrow B, 0 \leq t \leq 1$, be such an isotopy; that is, $h_{0}=g_{0}, h_{1}$ is $g_{1}$ or $g_{1} r$, and $h_{t}$ is an open embedding for each $t$. This isotopy induces a map $h: \mathbf{R}^{i} \times[0,1] \rightarrow B$ and we form the pull-back

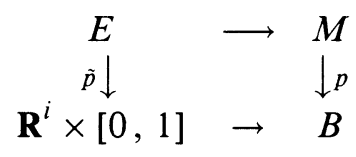

Clearly, the composition $p: E \stackrel{\tilde{p}}{\rightarrow} \mathbf{R}^{i} \times[0,1] \stackrel{\text { proj }}{\rightarrow}[0,1]$ is a submersion with open manifold fibres of dimension $\geq 5$. Since $\tilde{p} \mid: \tilde{p}^{-1}\left(\mathbf{R}^{i} \times\{t\}\right) \rightarrow \mathbf{R}^{i} \times\{t\}$ is an approximate fibration for each $t$ in $[0,1]$, we can use Siebenmann's Technical Bundle Theorem [27, Theorem 1.1, p. 60], to conclude that $p: E \rightarrow[0,1]$ is a fibre bundle projection (see [22, Lemmas 4.1 and 8.1]). 
Now Corollary 14.5 implies that $\tilde{p}_{0}$ and $\tilde{p}_{1}$ are controlled homeomorphic. The first part follows immediately. If $B$ is nonorientable, then $g_{0}$ is isotopic to both $g_{1}$ and $g_{1} \circ r$. The second part now also follows.

\section{AN $l_{2}$-FREE DESCRIPTION OF $M A F(B)$}

We show that the particular embedding of the total space of a manifold approximate fibration over $B$ in $l_{2} \times B$ is unimportant, at least up to homotopy. Specifically, we show that $M A F(B)$ is homotopy equivalent to the simplicial set $M A F_{*}(B)$, where the $k$-simplices of $M A F_{*}(B)$ are equivalence classes of $k$-simplices in $M A F(B)$. Two $k$-simplices in $M A F(B)$, say $M \subset l_{2} \times B \times \Delta^{k}$ and $N \subset l_{2} \times B \times \Delta^{k}$, represent the same $k$-simplex in $M A F_{*}(B)$ provided that there is a homeomorphism $h: M \rightarrow N$ which is fibre-preserving over $B \times \Delta^{k}$. It is not hard to see that $M A F_{*}(B)$ is Kan.

Theorem 15.1. The quotient map $\alpha: M A F(B) \rightarrow M A F_{*}(B)$ is a homotopy equivalence.

Proof. It is clear that $\alpha$ induces a surjection on homotopy groups. To see that $\alpha$ induces an injection on homotopy groups it suffices to consider the following situation. Let $M \subset l_{2} \times B \times \Delta^{k}$ and $N \subset l_{2} \times B \times \Delta^{k}$ represent classes in $\pi_{k}(M A F(B))$ with projections $p: M \rightarrow B \times \Delta^{k}$ and $q: N \rightarrow B \times \Delta^{k}$ such that $M$ and $N$ represent the same $k$-simplex in $M_{A} F_{*}(B)$. We need to show that $M$ and $N$ represent the same class in $\pi_{k}(M A F(B))$. By assumption there is a homeomorphism $h: M \rightarrow N$ such that $p=q \circ h$. Let $M(h)$ be the mapping cylinder of $h$ and let $\pi: M \times[0,1] \rightarrow M(h)$ be the quotient map. Let $j: M(h) \rightarrow l_{2} \times B \times \Delta^{k} \times[0,1]$ be an embedding of small capacity and fibre-preserving over $\Delta^{k} \times[0,1]$, such that $j=\pi^{-1}$ on $\pi(M \times\{0\}) \cup$ $\pi\left(p^{-1}\left(B \times \partial \Delta^{k}\right) \times[0,1]\right)$ and $j=\mathrm{id}$ on $\pi(M \times\{1\})$. The image of $j$ is an object over $\Delta^{k} \times[0,1]$ which shows that $M$ and $N$ represent the same class in $\pi_{k}(M A F(B))$.

Similar results are true for the other simplicial sets of interest to us; $B u n(B)$, $H u r(B)$ and $\overline{M A F}(B)$.

\section{SOME RESULTS ON FIBRATIONS}

Lemma 16.1. Let $p: E \rightarrow B$ be a regular fibration, and let $E(p) \rightarrow B$ be the associated Hurewicz fibration. There is a fibre inclusion $E \subset E(p)$ and this inclusion is a strong fibre deformation retract.

Proof. The associated Hurewicz fibration to $p$ is the subset of $E \times B^{I}$ consisting of all pairs $(e, \lambda)$ such that $p(e)=\lambda(0)$. The map $E(p) \rightarrow B$ sends $(e, \lambda)$ to $\lambda(1)$. See e.g. Spanier [36, p. 99, Theorem 9]. The inclusion of $E$ into $E(p)$ just sends $e$ to $(e, \lambda)$ where $\lambda$ is the constant path at $p(e)$. 
Recall that $p$ is a fibration iff we can solve the following universal lifting problem:

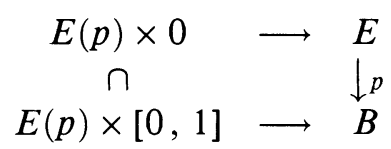

where the top map sends $(e, \lambda)$ to $e$ and the bottom map sends $((e, \lambda), t)$ to $\lambda(t)$. The fibration $p$ is a regular fibration iff we can find a solution $F: E(p) \times$ $[0,1] \rightarrow E$ to the above lifting problem with the additional property that $F((e, \lambda), t)=e$ whenever $\lambda$ is a constant path. See [11] for a proof that any fibration over a subset of $l_{2}$ must be regular.

Since we have a regular fibration, let $F$ denote a solution to the lifting problem with the regularity property. Define a map $\Phi: B^{I} \times[0,1] \rightarrow B^{I}$ by $\Phi(\lambda)(t)$ is the path defined by $\Phi(\lambda)(t)(s)=\lambda(t+(1-t) s)$. Next define $R: E(p) \times[0,1] \rightarrow E(p)$ by the formula $R((e, \lambda), t)=(F((e, \lambda), t), \Phi(\lambda)(t))$. The required properties are easily verified.

Lemma 16.2. Let $p: M \rightarrow B$ be given, and suppose that $B=B_{1} \cup B_{2}$, so that the pair $\left(B, B_{1} \cap B_{2}\right)$ is a strong NDR-pair and each $B_{i}$ is closed in $B$. Let $M_{i}=p^{-1}\left(B_{i}\right)$ and let $p_{i}: M_{i} \rightarrow B_{i}$ denote the restriction of $p, i=0,1,2$. Further suppose that $p_{i}$ is a fibration, $i=1,2$. Then $p$ is a fibration.

Proof. We will prove that $p$ is a fibre retract of its associated Hurewicz fibration. This easily proves the result. It also suffices to prove that $p$ restricted to a numerable open cover is a fibration, so we may assume without loss of generality that $\left(B, B_{i}\right)$ and $\left(B_{i}, B_{1} \cap B_{2}\right)$ are strong deformation retracts for $i=1,2$.

Let $r_{t}^{i}: B \rightarrow B$ denote the strong deformation retraction to $B_{i}$. By Strøm [37], we can cover $r_{t}^{i}$ by a deformation retraction $R_{t}^{i}: M \rightarrow M$ of $M$ to $M_{i}$. We can apply Strøm's results to the pair $\left(M_{j}, M_{0}\right)$ and then extend the deformation via the identity to $\left(M, M_{i}\right)$.

Let $E \rightarrow B$ be the associated Hurewicz fibration to $p$, and let $E_{i} \rightarrow B_{i}$ denote the associated Hurewicz fibration to $p_{i}$. We first construct a fibrewise strong deformation retraction from $E \mid B_{i}$ to $E_{i}$. Now $E \mid B_{i}$ is the subset of $M \times B^{I}$ which consists of all pairs $(m, \lambda)$ such that $p(m)=\lambda(0)$ and $\lambda(1) \in B_{i}$. Define a homotopy $\left(R_{t}^{i}(m), r_{t}^{i}(\lambda(s))\right)$. This keeps $E \mid B_{i}$ inside itself and defines a fibrewise strong deformation retract of $E \mid B_{i}$ into $E_{i}$. Since $p_{i}$ is a fibration, there is a fibre deformation retract of $E_{i}$ onto $M_{i}$ by Lemma 16.1. We can compose these to get a deformation $H_{t}^{i}: E\left|B^{i} \times[0,1] \rightarrow E\right| B_{i}$ which is a strong deformation retract onto $M_{i}$. In particular, we get strong fibrewise retracts of $E \mid B_{i}$ into $M_{i}, i=1,2$.

If the retract for $i=1$ agreed with the retract for $i=2$ when restricted to $E \mid B_{0}$ we would be done. This may not be the case, but they are fibre homotopic. Let us fix our retract on $E \mid B_{1}$, say $D^{1}$ and see what we can do on $E \mid B_{2}$.

Now $D^{1}$ restricted to $E \mid B_{0}$ is fibre homotopic to our retraction on $E \mid B_{2}$ restricted to $E \mid B_{0}$. The fibre homotopy can also be chosen to be the identity 
on $M_{0}$. Use the fibre-homotopy extension theorem to get a new map $K: E \mid B_{2} \rightarrow$ $E \mid B_{2}$ which is $D^{1}$ when restricted to $\mid B_{0}$.

We can glue $K$ and $D^{1}$ together to get a map $H: E \rightarrow E$. The image of $H$ is contained in the subspace $M_{1} \cup E \mid B_{2}$ of $E$ and is the identity on $M$. Moreover, $H$ is a fibre map. Define a map $J: M_{1} \cup E\left|B_{2} \rightarrow M_{1} \cup E\right| B_{2}$ by requiring it to be the identity on $M_{1}$ and our original retraction on $E \mid B_{2}$. It is clear that $J$ is continuous, a fibre map, and that restricted to $M \subset M_{1} \cup E \mid B_{2}$, $J$ is the identity. Furthermore, it is clear that the range of $J$ is $M$.

Then $J \circ K$ is the required fibre retract.

Lemma 16.3. Let $p: E \rightarrow B$ and $\pi: B \rightarrow X$ be given. Suppose that $p$ and $g=\pi \circ p$ are fibrations. Define $E_{\pi}(p) \subset E \times B^{I}$ by $(e, \lambda)$ is in $E_{\pi}(p)$ iff $p(e)=\lambda(0)$ and the path $\pi \circ \lambda$ on $X$ is a constant path. Then the evaluation map at $1, E_{\pi}(p) \rightarrow B$, is a fibration (called the $\pi$-associated Hurewicz fibration).

Proof. Let $B^{\pi} \subset B^{I}$ denote the space of paths on $B$ such that the path $\pi \circ \lambda$ on $X$ is a constant path. There is an evaluation map $B^{\pi} \rightarrow X$ and this map is a fibration since it is just the pull-back over the constant paths of the map $B^{I} \rightarrow X^{I}$, and this is a fibration since $B \rightarrow X$ is [36, p. 104, Exercise E.3].

We begin with a lifting problem

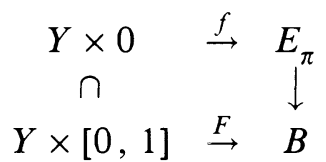

and proceed to solve it in stages.

The first step is to notice that our map $E_{\pi}(p) \rightarrow B$ maps to the map $E \rightarrow$ $X$, and since this is a fibration, we can find a solution $F_{1}: Y \times[0,1] \rightarrow E$ extending our map on $Y \times 0$. There is a similar map to $B^{\pi} \rightarrow X$ and hence a similar solution $L_{1}: Y \times[0,1] \rightarrow B^{\pi}$ extending our lift on $Y \times 0$. As a first approximation to our solution we take $F_{1} \times L_{1}: Y \times[0,1] \rightarrow E \times B^{\pi}$. There are only two things wrong with this attempt. The first is that the map need not land in $E_{\pi}(p)$ and the second is that it need not cover our original map into $B$. We repair these defects in two steps.

Let $\bar{F}_{1}: Y \times[0,1] \rightarrow E \rightarrow B$ and $\bar{L}_{1}: Y \times[0,1] \rightarrow B^{\pi} \rightarrow B$ where the first map is the obvious composite and the second is the composite of $L_{1}$ and evaluation at 0 . These two maps agree when restricted to $Y \times 0$ and when composed with the map $B \rightarrow X$. By [36, p. 101, Corollary 11], $\bar{F}_{1}$ and $\bar{L}_{1}$ are fibre homotopic rel $Y \times 0$. We can adjoint the homotopy to get a map $H: Y \times[0,1] \rightarrow B^{\pi}$ such that, on $Y \times 0, H$ gives the constant path at $\bar{F}_{1}$ (or equivalently at $\bar{L}_{1}$ ). The paths given by $H$ start at $\bar{F}_{1}$ and end at $\bar{L}_{1}$.

Define a new map $H * L_{1}: Y \times[0,1] \rightarrow B^{\pi}$ by the following formula:

$$
\left(H * L_{1}\right)(y, t)(s)= \begin{cases}H(y, t)\left(\frac{2 s}{t}\right), & 0 \leq s \leq \frac{t}{2}, \\ L_{1}(y, t)\left(\frac{2 s-t}{2-t}\right), & \frac{t}{2} \leq s \leq 1\end{cases}
$$


When restricted to $Y \times 0, H * L_{1}$ is just $L_{1}$; in general, $H * L_{1}$ gives a path that begins at $\bar{F}_{1}$ and ends at $\bar{L}_{1}$. Let $G=H * L_{1}$.

We try again. Consider the map $\bar{F}_{1} \times G: Y \times[0,1] \rightarrow E \times B^{\pi}$. This map lands in $E_{\pi}(p)$ and on $Y \times 0$ it is our original lift. Unfortunately, it is still not the case that we have lifted our original map $F$. Let $\bar{G}: Y \times[0,1] \rightarrow E_{\pi}(p) \rightarrow B$ denote the obvious composite. The notation is not bad because the above composite really does only depend on $G$. It is the case that $F$ and $\bar{G}$ agree when projected into $X$ via $\pi: B \rightarrow X$. Moreover, $F$ and $\bar{G}$ agree when restricted to $Y \times 0$. Just as above, there is a fibre homotopy from $\bar{G}$ to $F$ which adjoints to give a map $K: Y \times[0,1] \rightarrow B^{\pi}$ which yields constant paths when restricted to $Y \times 0$.

Now consider the map $G * K: Y \times[0,1] \rightarrow B^{\pi}$ defined by the formula:

$$
(G * K)(y, t)(s)= \begin{cases}G(y, t)\left(\frac{2 s}{2-t}\right), & 0 \leq s \leq 1-\frac{t}{2} \\ K(y, t)\left(\frac{2 s+t-2}{t}\right), & 1-\frac{t}{2} \leq s \leq 1\end{cases}
$$

This map is just $G$ when restricted to $Y \times 0$, and $G * K$ composed with the evaluation at $s=1$ is just our original map $F$. Also, $G * K$ composed with the evaluation at $s=0$ is given by $G$ evaluated at 0 ; which is $H$ evaluated at $s=0$; which is $\bar{F}_{1}$. Now, the map $F_{1} \times(G * K): Y \times[0,1] \rightarrow E \times B^{\pi}$ has all the desired properties.

\section{THE CONSTRUCTIONS OF FILL-INS}

Next we turn to the construction of fill-ins for Hur. The key to success is a construction of Tulley's which we recall.

(17.1) The Tulley Construction. We begin with two spaces over $B \times X$, say $p_{i}: E_{i} \rightarrow B \times X$ and a fibre map $f: E_{0} \rightarrow E_{1}$ which is an embedding. Each $E_{i}$ is also a subspace of $l_{2} \times B \times l_{2}$. We form the following subspace of $l_{2} \times B \times$ $X \times[0,1] \times l_{2}$ : in $l_{2} \times B \times X \times\left[\frac{1}{2}, 1\right] \times l_{2}$ take $f\left(E_{0}\right) \times \frac{1}{2} \cup E_{1} \times\left(\frac{1}{2}, 1\right]$ and in $l_{2} \times B \times X \times\left[0, \frac{1}{2}\right] \times l_{2}$ take the subset which is $E_{0}$ over $l_{2} \times B \times X \times 0 \times l_{2}$ and $f\left(E_{0}\right)$ over $l_{2} \times B \times X \times\left(0, \frac{1}{2}\right] \times l_{2}$. Let us use $T P(f)$ to denote this subset. There is also a map $T P(f) \rightarrow B \times X \times[0,1]$ given by applying either $p_{0}$ or $p_{1}$, whichever is appropriate. Denote this map by $T P(p)$. Notice that if $A \subset B$, we can restrict the above construction to $A$. Clearly $T P(f), \operatorname{TP}(p)$ when restricted to $l_{2} \times A \times X \times[0,1] \times l_{2}$ is just $T P(f \mid A)$ with its associated $T P(p)$, where $\left.f\right|_{A}$ is the map induced by $f$ from $p_{0}^{-1}(A)$ to $p_{1}^{-1}(A)$. Finally, notice that if we are also given a third map $p_{2}: E_{2} \rightarrow B \times X$, and fibre maps $f_{i}: E_{i} \rightarrow E_{2}, i=0,1$, such that $f_{1}$ is fibre homotopic to $f_{0} \circ f$, then we can also find a fibre map $F: T P(f) \rightarrow E_{2} \times[0,1]$ such that $F$ restricted over $B \times X \times 0$ is $f_{0}$ and $F$ restricted over $B \times X \times 1$ is $f_{1}$.

The next result follows from Tulley's proof of Theorem 4 in [38, pp. 613614]. 
Lemma 17.2. If the map $f$ above is a fibrewise strong deformation retract and $p_{1}$ is a fibration, then $T P(p): T P(f) \rightarrow B \times X \times[0,1]$ is a fibration.

We can now prove

Lemma 17.3. Hur satisfies the following version of the Fill-in Property. Suppose given three objects $E_{0}, E_{1}, E_{2}$ in $\operatorname{Hur}(B \times X)$, and two maps $f_{i}: E_{i} \rightarrow E_{2}$, $i=0,1$. Suppose that $B \times X$ is metric, so all fibrations are regular. Let $\bar{E}_{2}$ denote the result of pulling $E_{2}$ back to $B \times X \times[0,1]$ via the projection to $B \times X \times 0$. We can find an object $\bar{E}_{1}$ over $B \times X \times[0,1]$ such that $\bar{E}_{1}$ restricted over 0 is $E_{0}$ and $\bar{E}_{1}$ restricted over 1 is $E_{1}$. Moreover, we require $a$ map $F: \bar{E}_{1} \rightarrow \bar{E}_{2}$ such that $F$ restricted over 0 is $f_{0}$ and $F$ restricted over 1 is $f_{1}$. The construction is mildly functorial. Specifically, suppose we are given another set of initial data, say three objects $\widehat{E}_{0}, \widehat{E}_{1}, \widehat{E}_{2}$ over $B \times X$, and two maps $\hat{f}: \widehat{E}_{i} \rightarrow \widehat{E}_{2}, i=0,1$, and suppose that $\widehat{E}_{i}$ and $E_{i}$ are equal when restricted to $A \times X, A$ a subset of $B$. Moreover, suppose that $\hat{f}_{i}$ and $f_{i}$ agree when restricted to $A \times X$. Then the two extensions and the two extended maps agree when restricted to $A \times X \times[0,1]$.

Proof. We begin with our three fibrations as in the statement of the Fill-in Property. Since all our maps are fibre homotopy equivalences, we can find a fibre homotopy equivalence, $f: E_{0} \rightarrow E_{1}$, such that $f_{1}$ is fibre homotopic to $f_{0} \circ f$. If $f$ could be chosen to be an embedding and a strong fibrewise deformation retract, Lemma 17.2 would complete the proof. Unfortunately, this need not be the case, so the proof will consist in factoring our initial data into a sequence of situations in which we can apply the Tulley construction and Lemma 17.2. There is an embedding $l_{2} \subset l_{2}$ which sends the $i$ th coordinate to the $(i+1)$ st and is 0 in the 0 th coordinate. We first use the Tulley construction to replace both our $E_{i}$ 's in $l_{2} \times B \times X$ by copies where the 0th coordinate in the first $l_{2}$ is identically 0 .

Next we consider another fibration $l_{2} \times E_{1} \rightarrow B \times X$ which is just the projection to $E_{1}$ followed by the map $p_{1}$. As a subspace, it is naturally a subspace of $l_{2} \times l_{2} \times B \times X$ and we can embed $l_{2} \times l_{2}$ in $l_{2}$ so that the 0 th coordinate stays 0 and take the image. Let $\rho: E_{0} \subset l_{2} \times B \times X \rightarrow l_{2}$ denote projection onto the first factor. Then $\rho \times f: E_{0} \rightarrow l_{2} \times E_{1}$ is an embedding, as is the inclusion $E_{1} \rightarrow l_{2} \times E_{1}$ which sends $e \in E_{1}$ to $(0, e)$. The second of these maps is a strong fibrewise deformation retract, since $l_{2}$ is contractible rel 0 . Hence we may assume that $f$ is an embedding.

Next we can use Lemma 16.1 and Lemma 17.2 to replace each $E_{i}, i=0,1$, by its associated Hurewicz fibration. Each associated Hurewicz fibration is a subset of $E_{i} \times(B \times X)^{I}$ and the associated map is just the product of $f$ and the identity on $(B \times X)^{I}$. It is easy to check that this map is also an embedding. Let $E\left(p_{i}\right)$ denote the associated Hurewicz fibration to $p_{i}$ and let $E(f)$ denote the induced map on the associated Hurewicz fibrations. 
Let $\widehat{M}(f)$ denote the following subset of $E_{1} \times[0,1]: \quad \widehat{M}(f)=f\left(E_{0}\right) \times$ $[0,1) \cup E_{1} \times 1$. Furthermore, there is a map $\hat{p}: \widehat{M}(f) \rightarrow B \times X$ defined as $p_{1}$ on $E_{1} \times 1$ and $p_{0}$ otherwise. We make $E_{1} \times[0,1]$ into a subset of $l_{2} \times B \times l_{2}$ by letting the 0th coordinate of the first $l_{2}$ (which is 0 for $E_{1}$ ) be $z$ for $E_{1} \times z$. We can do the same construction for the associated Hurewicz fibrations and one easily checks that $E(\hat{p})=\widehat{M}(E(f))$.

There is an obvious strong deformation retract of $\widehat{M}(f)$ onto $E_{1} \times 1$, which is fibrewise. By applying this remark to $\widehat{M}(E(f))=E(\hat{p})$ and then applying the Tulley construction and Lemma 17.2, we are reduced to the case where one of our fibrations is $E\left(p_{0}\right)$; the other is $\widehat{M}(E(f))$; and the map is the composite of $E\left(p_{0}\right)$ into $E(f)\left(E\left(p_{0}\right)\right) \times 0$ on into $\widehat{M}(E(f))$.

If we can show that this last map is a fibrewise strong deformation retract, then we are done. We begin to do this by studying the map $E_{0}$ into the "top" of $\widehat{M}(f)$. This map is a fibre homotopy equivalence as well as an embedding. Suppose for now that we can prove that the "top" of $\widehat{M}(f)$ is a strong deformation retract.

Since $E(\hat{p})=\widehat{M}(E(f))$, we can use the strong deformation retract on $\widehat{M}(f)$ to construct a strong fibrewise deformation retract as follows. Let $R_{t}: \widehat{M}(f) \rightarrow$ $\widehat{M}(f)$ denote the strong deformation retract we are assuming exists. Composition with $\hat{p}$ defines a homotopy which adjoints to a map $r: \widehat{M}(f) \rightarrow B^{I}$. Since the deformation is strong, $r$ of any point in $f\left(E_{0}\right)$ (the "top") is a constant path. Let $\rho: \widehat{M}(f) \rightarrow[0,1]$ denote the evident projection, and note that the "top" is just $\rho^{-1}(0)$. Define a map $\widehat{M}(f) \times B^{I} \times[0,1] \rightarrow \widehat{M}(f) \times B^{I}$ by the formula $(m, \lambda, t) \rightarrow\left(R_{t}(m), \xi(m, \lambda, t)\right)$, where $\xi(m, \lambda, t)$ is the path given by the formula

$$
\xi(m, \lambda, t)= \begin{cases}r(m)\left(\frac{2 s}{\rho(m)}\right), & 0 \leq s \leq \frac{\rho(m)}{2}, \\ \lambda\left(t \frac{2 s-\rho(m)}{2-\rho(m)}\right), & \frac{\rho(m)}{2} \leq s \leq 1 .\end{cases}
$$

One can check that if we restrict this map to $E(\hat{p}) \times[0,1]$, the image is contained in $E(\hat{p})$ and the map defines a fibrewise strong deformation retract of $E(\hat{p})$ to its "top".

We return to the point that we omitted above, namely to show that $\rho^{-1}(0)$ is a strong deformation retract of $\widehat{M}(f)$. To do this we first remark that $\left(\widehat{M}(f), \rho^{-1}(0)\right)$ is an NDR-pair. The function that we want is not $\rho$, but rather $j(m)=\max (2 \rho(m), 1)$. With $j$ as the function, the required deformation is easily constructed. It is now a standard argument (e.g. [36, pp. 27-33]) to construct the required strong deformation retract from the homotopy equivalence between $f\left(E_{0}\right)$ and $E_{1}$.

The lemma is nearly proved. We need to check two further points. We have not been very careful to check that we can carry our maps to $E_{2} \times[0,1]$ along with us, but since we have checked this in the Tulley construction, this 
presents no difficulty for the diligent reader. The second point is to check that the answers for two different sets of starting data which are equal over some subspace of $B \times X$ of the form $A \times X$ are equal when restricted over $A \times X$. Again this is merely a matter of diligence although it might be useful to point out that the Tulley construction in no way uses the strong fibrewise deformation retract. It is merely used to prove that some map is a fibration.

To construct fill-ins for MAF requires yet another construction. This time we have three objects in $\operatorname{MAF}(B \times X)$, say $M_{0}, M_{1}$ and $M_{2}$, and controlled homeomorphisms $h_{i}: M_{i} \rightarrow M_{2}$ for $i=0,1$. We have suppressed the parameter $t$ in our notation. It is easy to get a controlled homeomorphism $h: M_{0} \rightarrow M_{1}$ such that $h_{0}=h_{1} \circ h$. The object we will use to "fill-in" is constructed as follows: $\widehat{M}(h)$ is the manifold obtained by gluing $M_{0} \times[0,1)$ to $M_{1} \times(0,1]$ using the map $h$ restricted to $M_{0} \times(0,1)$, where we have chosen the parameter interval to be $(0,1]$. There is a projection $p: \widehat{M}(h) \rightarrow B \times X \times[0,1]$ defined on $M_{1} \times(0,1]$ as the obvious projection and on $M_{0} \times[0,1)$ as $p_{1} \circ h(t)$ for $0<t<1$, and as $p_{0}$ for $t=0$. If we compose this map with the projection to $X \times[0,1]$, the map $\widehat{M}(h) \rightarrow X \times[0,1]$ is a bundle map. Clearly, $p$ restricted to each copy of $b \times X \times[0,1]$ is an approximate fibration. Both $M_{0}$ and $M_{1}$ are subsets of $l_{2} \times B \times X:$ let $e_{i}: M_{i} \rightarrow l_{2}$ denote the projection. Then Image $\left(e_{i}\right) \subset l_{2}$ has small capacity. Since $\widehat{M}(h)$ is a finite dimensional manifold, we can embed it in $l_{2}$, by $\hat{e}$, so that $\operatorname{Image}\left(e_{0}\right) \cup \operatorname{Image}\left(e_{1}\right) \cup \operatorname{Image}(\hat{e})$ has small capacity and such that $\left(\operatorname{Image}\left(e_{0}\right) \cup \operatorname{Image}\left(e_{1}\right)\right) \cap \operatorname{Image}(\hat{\boldsymbol{e}})=\varnothing$. There are retracts $r_{i}: \widehat{M}(h) \rightarrow M_{i}, i=0,1$, and projections $p_{I}: \widehat{M}(h) \rightarrow[0,1]$ and $p_{X}: \widehat{M}(h) \rightarrow X$. Define a map $E: \widehat{M}(h) \rightarrow l_{2} \times B \times X \times[0,1]$ by the formula $E(w)=\left(t e_{1}(w)+(1-t) e_{0}(w)+t(1-t) \hat{e}(w)\right) \times p_{X}(w) \times t$, where $t=p_{I}(w)$. Let $\bar{M}(h)$ denote the image of $\widehat{M}(h)$ under this embedding. Note that $\bar{M}(h)$ is an object in $\operatorname{MAF}(B \times X \times[0,1])$ which restricts to $M_{0}$ at one end and $M_{1}$ at the other.

Lemma 17.4. The functor MAF satisfies the Fill-in Property.

Proof. In the above paragraph, we constructed the objects we need. To complete the proof we need to check that we have the needed maps and the required uniqueness.

To define the needed maps first define a controlled homeomorphism $J_{t}: M_{0} \times$ $[0,1] \rightarrow M_{0} \times[0,1]$ for $0<t \leq 1$ by the formula

$$
J_{t}(m, s)= \begin{cases}(m, s), & t \geq s, \\ \left(h_{s}^{-1} \circ h_{t}(m), s\right), & t \leq s .\end{cases}
$$

We can use $J$ to construct a controlled homeomorphism $\widehat{J}: M_{0} \times[0,1] \rightarrow$ $\widehat{M}(h)$. Then our map $\widehat{M}(h) \rightarrow M_{2} \times[0,1]$ is given by the compcsite $h \circ \widehat{J}$. The required checks are straightforward. 
The uniqueness result is clear since the only choice we made in the construction was that of $\hat{e}$. Given two sets of data, we may embed the two $\widehat{M}(h)$ 's in $l_{2}$ so that they agree on the required subsets and are nonconstant in the same coordinates. The two resulting $\bar{M}(h)$ 's will then be equal on the required subsets, and the two maps will likewise be the same.

Lemma 17.5. The functor HAF satisfies the Fill-in Property.

Proof. The object we use is just the Tulley Construction as we did in the proof of Lemma 17.3 (Hur Fill-ins). Then we use Lemma 12.4 to change controlled homotopies to fibre homotopies and construct the required maps just as we did in the proof of Lemma 17.3. Details may be safely left to the reader.

\section{REFERENCES}

1. C. Allaud and E. Fadell, A fiber homotopy extension theorem, Trans. Amer. Math. Soc. 104 (1962), 239-251.

2. D. R. Anderson and W. C. Hsiang, The functors $K_{-i}$ and pseudo-isotopies of polyhedra, Ann. of Math. 105 (1977), 201-223.

3. D. Burghelea, On the decomposition of the automorphism groups, Rev. Roumaine Math. Pure Appl. 22 (1977), 17-30.

4. D. Burghelea, R. Lashof and M. Rothenberg, Groups of automorphisms of manifolds, Lecture Notes in Math., vol. 473, Springer-Verlag, New York, 1975.

5. T. A. Chapman, Controlled simple homotopy theory and applications, Lecture Notes in Math., vol. 1009, Springer-Verlag, New York, 1983.

6. __ Approximation results in topological manifolds, Mem. Amer. Math. Soc., no. 34 (1981).

7. D. Coram, Approximate fibrations-a geometric perspective, Shape Theory and Geometric Topology, edited by S. Mardesic and J. Segal, Lecture Notes in Math., vol. 870, SpringerVerlag, New York, 1981, pp. 37-47.

8. D. Coram and P. Duvall, Approximate fibrations, Rocky Mountain J. Math. 7 (1977), 275-288.

9. __ Approximate fibrations and a movability condition for maps, Pacific J. Math. 72 (1977), 41-56.

10. E. Dror, W. G. Dwyer and D. M. Kan, Automorphisms of fibrations, Proc. Amer. Math. Soc. 74 (1979), 183-186.

11. J. Dugundji, Topology, Allyn and Bacon, Boston, Mass., 1966.

12. __ Locally equiconnected spaces and absolute neighborhood retracts, Fund. Math. 57 (1965), 187-193.

13. R. D. Edwards, TOP regular neighborhoods, handwritten manuscript.

14. F. T. Farrell and W.-C. Hsiang, A structure set analogue of Chapman-Ferry-Quinn theory, Continua, Decompositions, Manifolds, University of Texas Press, Austin, 1983.

15. W.-C. Hsiang and R. W. Sharpe, Parametrized surgery and isotopy, Pacific J. Math. 67 (1976), 401-459.

16. S. T. Hu, Theory of retracts, Wayne State Univ. Press, Detroit, Mich., 1965.

17. C. B. Hughes, Approximate fibrations and bundle maps on Hilbert cube manifolds, Topology Appl. 15 (1983), 159-172.

18. _ Spaces of approximate fibrations on Hilbert cube manifolds, Comput. Math. 56 (1985), 131-151. 
19. Bounded homotopy equivalences of Hilbert cube manifolds, Trans. Amer. Math. Soc. 287 (1985), 131-151.

20. _ Approximate fibrations on topological manifolds, Michigan Math. J. 32 (1985), 167-183.

21. _ Delooping controlled pseudo-isotopies of Hilbert cube manifolds, Topology Appl. 26 (1987), 175-191.

22. _ Controlled homotopy topological structures, Pacific J. Math. 133 (1988), 69-97.

23. C. B. Hughes, L. R. Taylor and E. B. Williams, Controlled topology over manifolds of nonpositive curvature, preprint.

24. - Controlled surgery over manifolds, in preparation.

25. D. M. Kan, On homotopy theory and c.s.s. groups, Ann. of Math. 68 (1958), 38-53.

26. R. C. Kirby, Stable homeomorphisms and the annulus conjecture, Ann. of Math. 89 (1969), 575-582.

27. R. C. Kirby and L. C. Siebenmann, Foundational essays on topological manifolds, smoothings, and triangulations, Ann. of Math. Studies, no. 88, Princeton Univ. Press, Princeton, N.J., 1977.

28. J. P. May, Simplicial objects in algebraic topology, Van Nostrand Math. Studies, no. 11, Van Nostrand.

29. D. McDuff and G. Segal, Homology fibrations and the "Group Completion" theorem, Invent. Math. 31 (1976), 279-284.

30. D. Quillen, Homotopical algebra, Lecture Notes in Math., vol. 43, Springer-Verlag, New York, 1967.

31. F. Quinn, Ends of maps I, Ann. of Math. 110 (1979), 275-331.

32. __ Ends of maps II, Invent. Math. 68 (1982), 353-424.

33. __ Ends of maps III: dimensions 4 and 5, J. Differential Geometry 17 (1982), 503-521.

34. _ _ Homotopically stratified sets, J. Amer. Math. Soc. 1 (1988), 441-499.

35. __ Applications of topology with control, Proc. ICM Berkeley, 1986, pp. pp. 598-606.

36. E. Spanier, Algebraic topology, McGraw-Hill, 1966.

37. A. Strøm, Note on cofibrations II, Math. Scand. 22 (1968), 130-142.

38. P. Tulley, Strong homotopy equivalence and extensions for Hurewicz fibrations, Duke Math. J. 36 (1969), 609-619.

39. F. Waldhausen, Algebraic K-theory of generalized free products, Ann. of Math. 108 (1977), 135-256.

40. C. T. C. Wall, Surgery on compact manifolds, Academic Press, New York, 1970.

41. M. Weiss and E. B. Williams, Automorphisms of manifolds and algebraic $K$-theory, Part I, $K$-Theory 1 (1988), 575-626.

42. G. W. Whitehead, Elements of homotopy theory, Graduate Texts in Math. 61, SpringerVerlag, 1978.

Department of Mathematics, Vanderbilt University, Nashville, Tennessee 37235

Department of Mathematics, University of Notre Dame, Notre Dame, Indiana 46556 